\title{
An Assessment of the Role of Charged Secondaries from Nonelastic Nuclear Interactions by Therapy Proton Beams in Water
}

S. M. Seltzer

U.S. DEPARTMENT OF COMMERCE Technology Administration

National Institute of Standards and Technology

Gaithersburg, MD 20899

Prepared for:

National Cancer Institute Bethesda, MD 20892 



\section{An Assessment of the Role of Charged Secondaries from Nonelastic Nuclear Interactions by Therapy Proton Beams in Water}

\section{S. M. Seltzer}

U.S. DEPARTMENT OF COMMERCE Technology Administration National Institute of Standards and Technology

Gaithersburg, MD 20899

Prepared for:

National Cancer Institute Bethesda, MD 20892

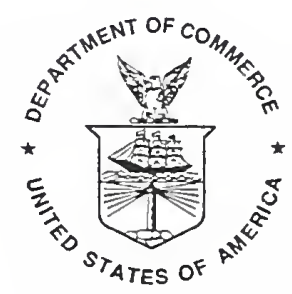

U.S. DEPARTMENT OF COMMERCE Ronald H. Brown, Secretary

NATIONAL INSTITUTE OF STANDARDS AND TECHNOLOGY

Arati Prabhakar, Director 


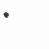




\title{
AN ASSESSMENT OF THE ROLE OF CHARGED SECONDARIES FROM NONELASTIC NUCLEAR INTERACTIONS BY THERAPY PROTON BEAMS IN WATER
}

\author{
Stephen M. Seltzer \\ National Institute of Standards and Technology \\ Gaithersburg, MD 20899
}

\begin{abstract}
This report summarizes the calculations and the syntheses of data performed to develop information on the cross sections in water for nonelastic nuclear interactions and the production of nuclear secondaries for protons with energies below $250 \mathrm{MeV}$. The data developed include the total nonelastic cross section and the number and energy distributions for secondary $\mathrm{n}, \mathrm{p}, \mathrm{d}, \mathrm{t},{ }^{3} \mathrm{He}, \alpha$, and some 32 recoiling nuclides produced in interactions of $p+{ }^{16} \mathrm{O}$. These data are used to evaluate slowing-down spectra (and pertinent moments) for the charged nuclear secondaries. Combining this information with primary proton fluence spectra obtained from Monte Carlo transport calculations, illustrative results are given for the average LET, as a function of depth, for unmodulated and modulated proton beams. Based on a crude biological-response model, the results are then used in exploratory calculations to estimate the relative biological effectiveness of the proton beams.
\end{abstract}




\section{Introduction}

For therapy proton beams, a significant fraction of the energy lost slowing down in water or tissue is due to nonelastic nuclear interactions with the target nuclei. In water, for example, this fraction is about 5 percent for $90-\mathrm{MeV}, 10$ percent for $140-\mathrm{MeV}$, and about 20 percent for $240-\mathrm{MeV}$ proton beams. For calculations of the spatial distribution of absorbed dose and fluence spectra from such beams, the nuclear interactions can be taken into account through knowledge of the total nonelastic cross section and a corresponding estimate of the effective fraction of the proton energy thereby converted to heavy-charged-particle kinetic energy that can be considered to be locally absorbed. The use of such data in the PTRAN (Berger, 1993) Monte Carlo calculations of primary proton transport is an example of this. Additionally, most of the secondary products from these nuclear interactions are densely ionizing and have a relative biological effectiveness larger than the incident protons. Comprehensive information on the production cross sections for the various products and on their energy (and angular) distributions would provide source terms for radiobiological modeling of the effects of these secondaries. Experimental data are far too sketchy to provide the desired information on the secondary particle spectra, but serve as important checks on results from available nuclear-reaction model calculations.

For applications involving water and tissue, it is necessary to model the nuclear interactions of protons with nuclei of $\mathrm{O}$ and $\mathrm{C}$ (and to a lesser extent, $\mathrm{N}$ ) over a range of proton kinetic energies from $250 \mathrm{MeV}$ down to a few $\mathrm{MeV}$. There is evidence that some light nuclei respond as if they contain preformed bound clusters of nucleons, particularly $\alpha$ particles in $\mathrm{C}$ and $\mathrm{O}$. It is said that inclusion of such cluster effects results in significant changes in the predictions of secondary particle production and of the distribution of the residual spallation products. It is therefore important that the calculation take this into account. The information desired are the production cross sections and differential spectra (in terms of energies and angles in the laboratory system) for the secondary particles $\mathrm{n}, \mathrm{p}, \mathrm{d}, \mathrm{t},{ }^{3} \mathrm{He}, \alpha$, and the recoiling residual nuclei. De-excitation gamma rays are also emitted as a result of the nuclear interaction, but - with a relative biological effectiveness assumed to be unity - are of less interest.

The synthesis of information on the nonelastic cross section represents an effort by someone who needs the information, rather than one who is an expert in the nuclear physics involved in this problem. Much advice was sought from qualified experts, including many of the developers of the model calculations used in this work. However, those individuals cannot be held responsible for possible errors in the results described here. Work has progressed far enough to develop some useful data for water. The remainder of this report is, therefore, concerened only with the $\mathrm{p}+{ }^{16} \mathrm{O}$ reactions.

\section{Sources Used for the Nuclear Data}

Some relevant data are available from earlier and current calculations. A number of sources have so far been considered that (a) are generally regarded as representative of good work, (b) at least partially fulfill our needs, and (c) which we have been able to acquire without a disproportionate level of effort. 
NCDATA Alsmiller et al. (1967) obtained analytical fits of the results generated by Bertini (1963, 1966) with his intranuclear-cascade/evaporation Monte Carlo calculations. The code NCDATA (Alsmiller and Barish, 1968) interpolates over these fits to give the total nonelastic cross section and the secondary neutron and proton spectra (in four angular intervals) from both the cascade and the evaporation stages of the calculation, for either neutrons or protons with energies from 25 to $400 \mathrm{MeV}$ incident on a nucleus with an atomic mass number between 12 and 238 . The code has been converted to run on a PC. The results fulfill only a portion of our needs. Moreover, the models used by Bertini ignore cluster effects and use statistical evaporation in the compound stage, perhaps not the best approaches for light nuclei. Janni (1982) adopted the Bertini results for the total nonelastic cross section for the energy range from 25 to $100 \mathrm{MeV}$, but switches to those of Wilson and Costner (1975) for $150 \mathrm{MeV}$ and above.

Townsend et al. Townsend et al. (1983) and Townsend and Wilson (1985) use eikonal scattering theory to calculate total and absorption (nonelastic) cross sections for nucleon and deuteron scattering from nuclei of $\mathrm{He}$ to $\mathrm{Pb}$. Their calculation makes no distinction between incident protons or neutrons, an approximation appropriate at high energies. This latter work supersedes the earlier calculations of Wilson and Costner (1975).

ALICE Blann (1991 and references therein) has developed over the last 20 years a generalpurpose code based on a hybrid exciton pre-equilibrium decay model and Weisskopf-Ewing evaporation model. The code has not been written to optimize accuracy for all reactions, but does allow for the selection among a number of model options governing the calculation. If not otherwise supplied, total nonelastic interaction cross sections are derived from an optical model calculation and used for the normalization of all partial cross sections. The code would seem capable of generating most of the desired information, except that (a) no spectra of the residual recoil nuclei are calculated; (b) the differential spectra that are calculated are given only in the center-of-mass (CM) system and - because two-body kinematics do not in general apply - would not be easy to convert to the laboratory system; and (c) the nuclear models used are probably not too reliable for light target nuclei. We have converted the version ALICE91 to run on the PC, and have generated our data with much helpful advice from Dr. Blann on the selection of the input and the interpretation of the output.

GNASH Young et al. (1990) have recently refined the GNASH code in the preparation of evaluated data libraries for incident neutron and proton energies up to $100 \mathrm{MeV}$. Included in this work are data sets for $\mathrm{C}$ and $\mathrm{O}$. Their calculation is based on an exciton model for pre-equilibrium decay and (mainly) a Hauser-Feshbach statistical model for decay of the compound nucleus. The code is considered an evaluator's tool and requires a level of expertise to run. The code was not acquired, but the final data sets for $\mathrm{C}$ and $\mathrm{O}$, and advice on their interpretation, were kindly provided by Dr. Young. For these data also, the differential spectra are given in the CM-system, and the code fragments sent us by Dr. Young to convert the data to the laboratory system do not run on our platform.

INCA-FBRK These codes were developed by Brenner et al. (1981) (see also Brenner and Prael, 1987, and references therein) and used to generate a data set for 15-60 MeV neutrons on C and O (Brenner and Prael, 1989). The first of the codes, INCA, is a Monte Carlo intranuclearcascade pre-equilibrium calculation derived from the VEGAS code of Chen et al. (1968), with refinements among which are the inclusion of $\alpha$ and two-nucleon clusters in the nucleus. The FBRK Monte Carlo code treats particle emission from the equilibrium compound nucleus according to a 
Fermi-breakup model rather than an evaporation model. The INCA code appears to be rather similar to the CLUST code (CC) of Mathews et al. (1982) on which it is based; the FBRK code was developed independently, but appears to have much in common with the Fermi breakup model (FBM) developed by Gökmen et al. (1984). In the LAHET code (Prael, 1989), the Brenner et al. Fermi breakup model is used for light nuclei; the default intranuclear cascade calculation is done with Bertini's code, but with optional use of the ISABEL/VEGAS code; and there is also included provision for the optional use of an intermediate multistage pre-equilibrium exciton calculation. Because of the differences among the refinements in the various codes, there is no assurance that the results from INCA-FBRK are in all respects equivalent to those from CC-FBM or from LAHET.

The effort in getting all of the Monte Carlo codes running on our own platforms would be significant. We have obtained only the INCA-FBRK codes and converted them to the PC, an effort possible only with the patient advise of Dr. Brenner. For most of our calculations, the nuclear density distribution was approximated by a 3-parameter Fermi model, with parameter values taken from de Jager et al. (1974), and then represented by a step function over 18 concentric shells. Neutron, proton, deuteron and $\alpha$-particle spectroscopic factors were from the work of Balashov et al. (1964). Cutoff energies, below which particles in the cascade calculation are assumed to be trapped in the target nucleus, were taken to be the binding energy for neutrons, the sum of the binding energy and 1.2 times the Coulomb barrier for protons and deuterons, and the sum of the binding energy and 1.5 times the Coulomb barrier for $\alpha$ particles. Occasional numerical problems, apparently associated with round-off due to the shorter wordlength on the PC, can cause a run to abort. This problem has been dealt with by simply changing the initial random number, but has had the effect of effectively limiting the number of histories we can consider in a calculation. The results from our INCA calculations are based on 100,000 incident protons; and the decays of the resulting excited compound nuclei were then followed 10 times each in FBRK.

Two artifacts were noticed in the spectra of secondary protons from our preliminary calculations. The results of INCA include a contribution from compound-elastic scattering which should not be counted in the nonelastic cross section. The recoiling ${ }^{16} \mathrm{O}$ nuclei from compound-(as well as from shape-) elastic scattering may be an important consideration, and should be added later. To remove the compound-elastic component from the results, the scores in the energy-angle bins corresponding to elastic scattering were zeroed out. However, it was further noticed that the highenergy portion of the secondary proton spectra extended up to energies beyond that allowed for p-p' scattering. To remove this unphysical contribution, the scores in energy bins corresponding to an energy loss less than the energy level of the first excited state of the target nucleus $(6.049,2.313$, and $4.439 \mathrm{MeV}$ in ${ }^{16} \mathrm{O},{ }^{14} \mathrm{~N}$, and ${ }^{12} \mathrm{C}$, respectively) were set to zero (which effectively also takes care of the compound-elastic component). This reduces the predicted total nonelastic cross section significantly, by about 20-25 percent at energies from 250 to $20 \mathrm{MeV}$ and by larger amounts at lower energies.

\section{3. $p+{ }^{16} \mathrm{O}$ Nonelastic Cross Sections}

\subsection{Total Nonelastic Cross Section}

Calculated values of the total nonelastic cross sections for $p+{ }^{16} \mathrm{O}$ are compared in figure 1a with measured values from Chapman and Macleod (1967), Carlson et al. (1975) and Renberg et al. (1972), found in the review of Bauhoff (1986). The various calculations tend to predict the cross 
section to within about 20 percent of the measured values. Interestingly, the results from NCDATA at energies above $100 \mathrm{MeV}$ are in better agreement with the experimental data than are the WilsonCostner cross sections adopted by Janni. The newer calculations of Townsend and Wilson are a considerable improvement over the earlier Wilson-Costner results. The GNASH results take into explicit account the threshold energies for the various inelastic exit channels, indicating that the total nonelastic cross section is effectively zero below about $6 \mathrm{MeV}$. Below this threshold, the cross sections adopted by Janni and those predicted by ALICE would then seem in error. With the adjustments regarding the $\mathrm{p}-\mathrm{p}^{\prime}$ cross section as mentioned above, the results from INCA-FBRK follow fairly well the measured data and the GNASH threshold behavior. The solid curve has been drawn as a fit to the experimental data, constrained by the high-energy asymptotic value for the cross section of approximately $310 \mathrm{mb}$ (see Letaw et al., 1983) and by the threshold indicated by the GNASH results. This adopted curve is plotted again in figure $1 \mathrm{~b}$ for a less cluttered comparison with the experimental data and the values used by Janni (1982); figure 1c gives a linear plot of the adopted cross section. The adopted cross section is used in the PTRAN calculations

With knowledge of the total nonelastic cross section, a straight-forward calculation (in the continuous-slowing-down approximation) can be done to obtain the fraction lost of the initial number and energy of protons slowing down in water. Such results are shown in figure 2, based on the adopted cross section.

Further results, for partial cross sections and distributions, have been obtained from the model calculations (mainly from INCA-FBRK). To facilitate the use of such information with preferred choices for the total cross section, the results have been normalized to one nuclear interaction. In those cases where absolute data are given, the results have been normalized to the adopted total cross section.

\subsection{Partial Cross Sections for Nuclear Secondary Particles}

Calculated multiplicities associated with the production of light secondary particles, from the calculations considered, are compared in table 1. Overall, the agreement is not too bad, perhaps reflecting the relative strengths and weaknesses of the different models.

The information obtained from the INCA-FBRK calculations, including that for the heavier recoiling nuclei, is given in table 2. The recoils for these cases can include over 30 nuclei. Comparisons of nuclide-production cross sections derived from ALICE and INCA-FBRK calculations with the rather sketchy experimental data compiled by Read and Viola (1984) and by Bernas et al. (1967) are given in figures 3a-k. Calculated values, normalized to the adopted total nonelastic cross section, were obtained for the incident proton energies listed in table 2 and simply connected by straight lines in figures 3 . Overall agreement appears to be within roughly a factor of two, but it seems difficult to choose among the calculated and experimental data. Beyond the purpose of code checking that concerns us here, there may be interest in the production of $\beta^{+}$-emitting nuclides such as ${ }^{11} \mathrm{C},{ }^{13} \mathrm{~N}$ and ${ }^{15} \mathrm{O}$ in regard to the use of positron-emission-tomography (PET) for the visualization of proton beams in phantom.

\subsection{Energy Transferred to Nuclear Secondary Particles}

The average fractions of the energy transferred to the kinetic energy of nuclear secondary particles, obtained from the INCA-FBRK calculations, are given in table 3 for $n, p, d, t,{ }^{3} \mathrm{He}, \alpha$, and recoils. Assuming the energy given to neutrons and gamma rays is not deposited in the region 
of interest, the fraction for all charged particles is of interest for the calculation of absorbed dose in an approximation in which secondary transport is not included. This fraction, assumed locally absorbed, is plotted in figure 4. The fitted curve shown in figure 4 is used in the PTRAN calculations of the absorbed-dose distributions by proton beams in water.

The energy spectra of the secondary particles are shown in figures 5a-q. These spectra, from the INCA-FBRK calculations, have been integrated over emission angle and normalized to one emitted particle of the type shown. The recoil spectra, plotted in figures $5 \mathrm{~g}-\mathrm{q}$, include all residual nuclei with the stated mass number. It appears that many of the spectra could be fitted with functions that are generally of the type $\mathrm{E}^{\mathrm{b}} \mathrm{e}^{-\mathrm{aE}}$, as suggested by LeCouteur (1952) for evaporation particles.

\section{Slowing-Down and LET Spectra of the Nuclear Secondaries}

The initial secondary spectra of figures 5 can be used as input in a slowing-down calculation. Let $\phi_{j}\left(T, T_{o}\right)$ be the initial spectrum, differential in emitted particle energy $T$, for the secondary of type $\mathrm{j}$ produced by a primary proton of energy $\mathrm{T}_{\mathrm{o}}$. We assume here that $\phi_{\mathrm{j}}\left(\mathrm{T}, \mathrm{T}_{\mathrm{o}}\right)$ is normalized to one nonelastic interaction (which requires the multiplication of the spectra in figures 5 by the multiplicity for particle j). In the continuous-slowing-down approximation, the mean tracklength, per unit energy, covered by particles of energy $T$ slowing down from energies between $T$ and $T_{0}$ is given by

$$
Y_{j}\left(T, T_{o}\right)=\frac{1}{S_{j}(T)} \int_{T}^{T_{o}} \phi_{j}\left(T^{\prime}, T_{o}\right) d T^{\prime}
$$

where $S_{j}(T)$ is the mass total stopping power (the sum of collision and nuclear stopping powers) for particle $\mathrm{j}$. The total dose $\mathrm{D}_{\mathrm{j}}\left(\mathrm{T}_{\mathrm{o}}\right)$ is simply

$$
D_{j}\left(T_{o}\right)=\int_{o}^{T_{o}} S_{j}(T) Y_{j}\left(T, T_{o}\right) d T .
$$

The distribution of LET is given by

$$
F_{j}(L) d L=L_{j}(T) Y_{j}\left(T, T_{o}\right) d T,
$$

where $L_{j}$ is the unrestricted linear energy transfer (i.e., the collision stopping power) for particle $j$. We avoid the necessity of smoothing and/or re-binning the spectral histograms for the determination of $F_{j}(L)$ and proceed directly to the calculation of the dose-averaged LET:

$$
\langle L E T\rangle_{\text {dose }}^{(j)}=\frac{1}{D_{j}\left(T_{o}\right)} \int_{o}^{T_{o}} L_{j}(T) Y_{j}\left(T, T_{o}\right) d T
$$

Note that we have ignored the higher-order effects of nuclear reactions by the secondary particles. 
Values for the mass stopping powers in water were obtained as follows. For protons and $\alpha$ particles, the stopping powers were taken directly from the new compilation in ICRU Report 49 (1993). For other secondaries with atomic numbers $Z$ of 1 and 2, the collision stopping powers for $\mathrm{p}$ and $\alpha$ from ICRU Report 49 were applied according to the velocity scaling relationship, and nuclear stopping powers were obtained from the TRIM92 code of Ziegler and Biersack (1992?). For all other ions, the stopping powers from TRIM92 were used. The stopping-power database for some 37 ions is shown graphically in figure 6 by plotting the stopping powers vs. the particle energy divided by its mass in atomic mass units. The curves, for $\mathrm{Z}$ from 1 to 8 , are actually families of curves mainly due to the departure from scaling of the nuclear stopping power. The lower set of curves, below about $0.05 \mathrm{MeV} / \mathrm{amu}$, represent the mass collision stopping powers.

Results for the slowing-down spectra for secondary particles from $100-\mathrm{MeV}$ primary protons are shown in figures 7a-p. The calculated values of the dose-averaged LET for nuclear secondaries from primary protons with energies up to 250 are given in table 4. Again note that further nuclear interactions and the production of tertiaries, etc., are ignored. The calculated LET values averaged over all secondary charged particles is plotted in figure 8 along with a fitted curve. The steep rise shown near threshold is of course very uncertain, but is mitigated by the sharp drop in the corresponding dose shown in figure 4.

These results can be combined with the differential tracklength distributions for the primary protons obtained from recent PTRAN calculations (Berger, 1992). Distributions of dose-averaged LET as a function of depth are shown in figures 9a-c. These results, for 250-, 160-, and 70-MeV incident protons, are given both for the unmodulated beam and for a modulated beam assuming 21 equal-thickness absorber steps to spread the Bragg peak over depths of 20,10 , and $2.5 \mathrm{~cm}$, respectively. It is clear that in all of these examples the contribution by the nuclear secondaries to the average LET is quite significant at depths up to the Bragg peak, but that the primary protons slowing to low energies and high LET completely dominate the region beyond.

\section{Qualitative Estimates of Relative Biological Effectiveness}

Radiobiological studies of the effects of ionizing radiation on mammalian cells have accumulated over more than 30 years, along with our appreciation of the complexities involved in understanding them. The relative biological effectiveness (RBE) of a radiation has usually been determined as the ratio of the dose of a reference low-LET radiation to that of the high-LET radiation that is necessary to produce the biological effect under investigation, both obtained from measured dose-effect curves. In addition to the variability of the systems and endpoints studied, such determinations of RBE are further complicated by the choice of the measured level of effect (or of some parametric quantity extracted from the analysis of dose-effect curves), by the biochemical environment of the system (e.g., oxygen-enhancement ratios), and perhaps by incomplete knowledge of the radiation field incident on the system.

In spite of such statements, we have constructed a naive overview to orient ourselves on the possible contributions to the RBE of therapy proton beams. Stated RBE's, relative to ${ }^{60} \mathrm{Co}$ irradiation, extracted from mammalian cell response for monoenergetic (or nearly so) ions have been collected from the literature. These are plotted simply as a function of particle LET in figure 10. Included are the data of (a) Powers-Riesius, as found in Shavers et al. (199?), from mouse 
spermatogonia cell survival for irradiation by $\mathrm{He}, \mathrm{C}, \mathrm{Ne}, \mathrm{Si}, \mathrm{Ar}, \mathrm{Fe}, \mathrm{Nb}$ and $\mathrm{La}$ ions (from which the RBE's have been renormalized to unity at low LET to correct to ${ }^{60} \mathrm{Co}$-equivalent); (b) Barendsen (1972) from human kidney cell survival for $\alpha$-particle and deuteron irradiation; (c) Matsubara et al. (1990) from induction of chromosome aberrations in bovine lymphocytes for proton irradiation; (d) Bettega et al. (1979) from human (EUE) cell survival for proton irradiation; (e) Raju et al. (1978) from human (T1) and Chinese hampster (V79) cell survival for irradiation by protons, $\alpha$ particles, and $\mathrm{C}, \mathrm{Ne}$ and Ar ions; and (f) Courdi et al. (1992) from human melanoma, breast carcinoma and neuroblastoma cell survival for irradiation by $\mathrm{O}, \mathrm{Ni}, \mathrm{Ne}, \mathrm{Ar}, \mathrm{Kr}$ and $\mathrm{Au}$ ions.

Drawn also in figure 10 are dashed curves to suggest possible envelopes for the data. A functional form for the curves was adopted from recommendations of the ICRP (1990) for the quality factor used in radiation protection. We have assumed

$$
\operatorname{RBE}(\mathrm{L})=\left\{\begin{array}{cc}
1 & \mathrm{~L} \leq \mathrm{L}_{1} \\
\mathrm{aL}-\mathrm{b} & \mathrm{L}_{1}<\mathrm{L}<\mathrm{L}_{2} \\
\frac{\mathrm{c}}{\sqrt{\mathrm{L}}} & \mathrm{L} \geq \mathrm{L}_{2}
\end{array}\right.
$$

where

$$
\begin{aligned}
& \mathrm{a}=\frac{\mathrm{RBE}_{\max }-1}{\mathrm{~L}_{2}-\mathrm{L}_{1}} \\
& \mathrm{~b}=\mathrm{aL}_{1}-1 \\
& \mathrm{c}=\mathrm{RBE}_{\max } \sqrt{\mathrm{L}_{2}}
\end{aligned}
$$

and $R_{B E}$ is the peak value of the RBE (at an LET equal to $L_{2}$ ). The value $L_{2}=100 \mathrm{keV} / \mu \mathrm{m}$ has been chosen for both curves. For the lower curve, $\mathrm{L}_{1}=10 \mathrm{keV} / \mu \mathrm{m}$ and $\mathrm{RBE}_{\max }=3$; for the upper curve, $\mathrm{L}_{1}=1.5 \mathrm{keV} / \mu \mathrm{m}$ and $\mathrm{RBE}_{\max }=6$. This simplistic functional form is a reminder of our lack of information beyond a crude level; the discontinuities in the curves should not be taken seriously.

Assuming such a response, the effective dose is evaluated according to

$$
D_{j}^{e f f}\left(T_{o}\right)=\int_{0}^{T_{0}} R B E\left[L_{j}(T)\right] S_{j}(T) Y_{j}\left(T, T_{o}\right) d T,
$$

and the average RBE is the ratio of the effective dose to the physical dose from Eq. 2. Results of this calculation, averaged over all nuclear secondaries, are plotted in figure 11, along with smooth curves fitted to calculated points. The "high" and "low" curves correspond, respectively, to the use of the upper and lower curves in figure 10.

These results can be combined with corresponding results for the primary proton tracklength distributions from PTRAN calculations to obtain estimates of the RBE of proton beams as a function of depth in a water phantom. Figures 12a-c show such calculated RBE's for protons with initial 
energies of 250,160 and $70 \mathrm{MeV}$, for unmodulated and modulated beams. An expanded plot of the results in the distal region of the Bragg peak for $160-\mathrm{MeV}$ beams are shown in figure 13 along with RBE's measured by Robertson et al. (1975) from the analysis of rat hepatoma cell survival. Other measurements in modulated $160-\mathrm{MeV}$ beams give RBE values of (a) 1.19-1.23 in the spread-out Bragg peak (SOBP), and 1.13-1.17 in the valley region (Tepper et al., 1977); (b) 1.20 in the SOBP and 1.17 in the valley at high doses (Hall et al., 1978); and (c) 1.03-1.42 in the SOBP and valley (Urano et al., 1980). Robertson et al. (1993) recently report RBE's from 1.05 to 1.20 for a $64-\mathrm{MeV}$ beam, and from 1.2 to 1.3 for $200-\mathrm{MeV}$ beams. Wainson et al. (1972) report an RBE at the Bragg peak of an unmodulated $90-\mathrm{MeV}$ beam that is 1.44 times that at smaller depths.

Although it appears that quantitative similarity with measured RBE values can be obtained in these cases, it must be recognized that the model used here is quite crude. In addition to other shortcomings, no attempt has been made to include the contribution by secondary neutrons, nor to take into account variations of RBE with dose level or dose rate. In regard to the latter, it is known that the RBE of high-LET particles tend to rise as the dose becomes smaller. This is, for example, reflected in the data of Barendsen and of Raju et al. in figure 10 which show a larger RBE when a higher surviving fraction (and hence a lower dose) is used. Hall et al. (1978) found a dose-level effect for the measured RBE from a $160-\mathrm{MeV}$ proton beam. Such an effect could suggest that, even using our crude model, one might move from the low-RBE curve (or lower) in figure 10 for the primary protons, which deliver a relatively high dose in clinical applications, toward the high-RBE curve (or higher) for the nuclear secondary component, which contributes a relatively low dose. 


\section{REFERENCES}

Alsmiller, R.G. Jr., Leimdorfer, M., and Barish J. (1967), Oak Ridge National Laboratory Report ORNL-4046.

Alsmiller, R.G. Jr. and Barish J. (1968), Oak Ridge National Laboratory Report ORNL-4220.

Balashov, V.V., Boyarkina, A.N., and Rotter I. (1964), Nucl. Phys. 59, 417.

Bauhoff, W. (1986), At. Data and Nucl. Data Tables, 35, 429.

Berger, M.J. (1993), National Institute of Standards and Technology Publication NISTIR 5113.

Bernas, R., Gradztajn, E., Reeves, H., and Schatzman, E. (1967), Ann. Phys. (NY) 44, 426.

Bertini, H.W. (1963), Phys. Rev. 131, 1801; errata in Phys. Rev. 138, AB2 (1965).

Bertini, H.W. (1966), Nucl. Phys. 87, 138.

Blann, M. (1991), International Atomic Energy Agency Publication INDC(NDS)-245, p. 63; and Lawrence Livermore National Laboratory Publication UCRL-JC-109052 (1991).

Brenner, D.J., Prael, R.E., Dicello, J.F., and Zaider, M. (1981), "Improved Calculations of Energy Deposition from Fast Neutrons", Proc. Fourth Symp. on Neutron Dosimetry, EUR-7448, Munich-Neuherberg.

Brenner, D.J. and Prael, R.E. (1987), in Nuclear and Atomic Data for Radiotherapy and Related Radiobiology, IAEA, Vienna, p. 205.

Brenner, D.J. and Prael, R.E. (1989), At. Data and Nucl. Data Tables, 41, 71.

Carlson, R.F., Cox, A.J., Nimmo, J.R., Davison, N.E., Elbakr, S.A., Horton, J.L., Houdayer, A., Sourkes, A.M., van Oers, W.T.H., and Margaziotis, D.J. (1975), Phys. Rev. C12, 1167.

Chapman, R. and Macleod, A.M. (1967), Nucl. Phys. A94, 313.

Chen, K., Fraenkel, Z., Friedlander, G., Grover, J.R., Miller, J.M., and Shimamoto, Y. (1968), Phy. Rev. 166, 949.

de Jager, C.W., de Vries, H., and de Vries, C. (1974), At. Data and Nucl. Data Tables, 14, 479.

Gökmen, A., Mathews, G.J., and Viola, V.E. Jr. (1984), Phys. Rev. C 29, 1606.

Janni, J.F. (1982), At. Data and Nucl. Data Tables 27, 147.

LeCouteur, K.J. (1952), Proc. Phys. Soc. A 65, 718. 
Letaw, J.R., Silberberg, R., and Tsao, C.H. (1983), Astrophys. J. Suppl. Ser. 51, 271.

Mathews, G.J., Glagola, B.G., Moyle, R.A., and Viola, V.E. Jr. (1982), Phys. Rev. C 25, 2181.

Prael, R.E. (1989), Los Alamos National Laboratory Publication LA-UR-89-3347.

Read, S.M. and Viola, V.E. Jr. (1984), At. Data and Nucl. Data Tables, 31, 359.

Renberg, P.U., Measday, D.F., Pepin, M., Schwaller, P., Favier B., and Richard-Serre C. (1972), Nucl. Phys. A183, 81.

Townsend, L.W. and Wilson, J.W. (1985), National Aeronautics and Space Administration Reference Publication 1134.

Townsend, L.W., Wilson, J.W. and Bidasaria, H.B. (1983), National Aeronautics and Space Administration Technical Memorandum 84636.

Wilson, J.W. and Costner, C.M. (1975), National Aeronautics and Space Administration Technical Note TN-8107.

Young, P.G., Arthur, E.D., Bozoian, M., England, T.R., Hale, G.M., LaBauve, R.J., Little, R.C., MacFarlane, R.E., Madland, D.G., Perry R.T., and Wilson, W.B. (1990), Los Alamos National Laboratory Publication LA-11753-MS. 
Table 1. Average multiplicities for light secondary particles from some available calculations for $p+{ }^{16} \mathrm{O}$. Numbers in parentheses are interpolated.

\begin{tabular}{|c|c|c|c|c|c|}
\hline & $\begin{array}{c}\text { Incident } \\
\text { Proton Energy } \\
(\mathrm{MeV})\end{array}$ & ALICE & NCDATA & INCA-FBRK & GNASH \\
\hline \hline $\mathrm{m}_{\mathrm{a}}$ & 250 & 1.47 & 1.35 & 0.77 & - \\
& 100 & 1.04 & 1.10 & 0.60 & 0.66 \\
& 30 & 0.15 & 0.54 & 0.16 & $(0.08)$ \\
\hline & 250 & 2.44 & 2.32 & 1.82 & - \\
$\mathrm{m}_{\mathrm{a}}$ & 100 & 2.05 & 1.96 & 1.57 & 1.55 \\
& 30 & 1.24 & 1.29 & 0.97 & $(1.16)$ \\
\hline & 250 & 0.65 & - & 0.10 & - \\
$\mathrm{m}_{\mathrm{a}}$ & 100 & 0.29 & - & 0.14 & 0.24 \\
& 30 & 0.06 & - & 0.15 & $(0.04)$ \\
\hline & 250 & 0.63 & - & 0.79 & - \\
$\mathrm{m}_{\alpha}$ & 100 & 0.51 & - & 0.91 & 0.86 \\
& 30 & 0.60 & - & 1.05 & $(0.63)$ \\
\hline & 250 & 5.19 & 3.67 & 3.58 & - \\
$\mathrm{m}_{\text {cot }} \cdot$ & 100 & 3.88 & 3.06 & 3.29 & 3.31 \\
& 30 & 2.05 & 1.83 & 2.34 & 1.91 \\
\hline
\end{tabular}

ALICE $(n+p+d+\alpha), \operatorname{NCDATA}(n+p), \operatorname{INCA}-F B R K\left(n+p+d+t+{ }^{3} H e+\alpha\right)$, GNASH $(n+p+d+\alpha)$ 


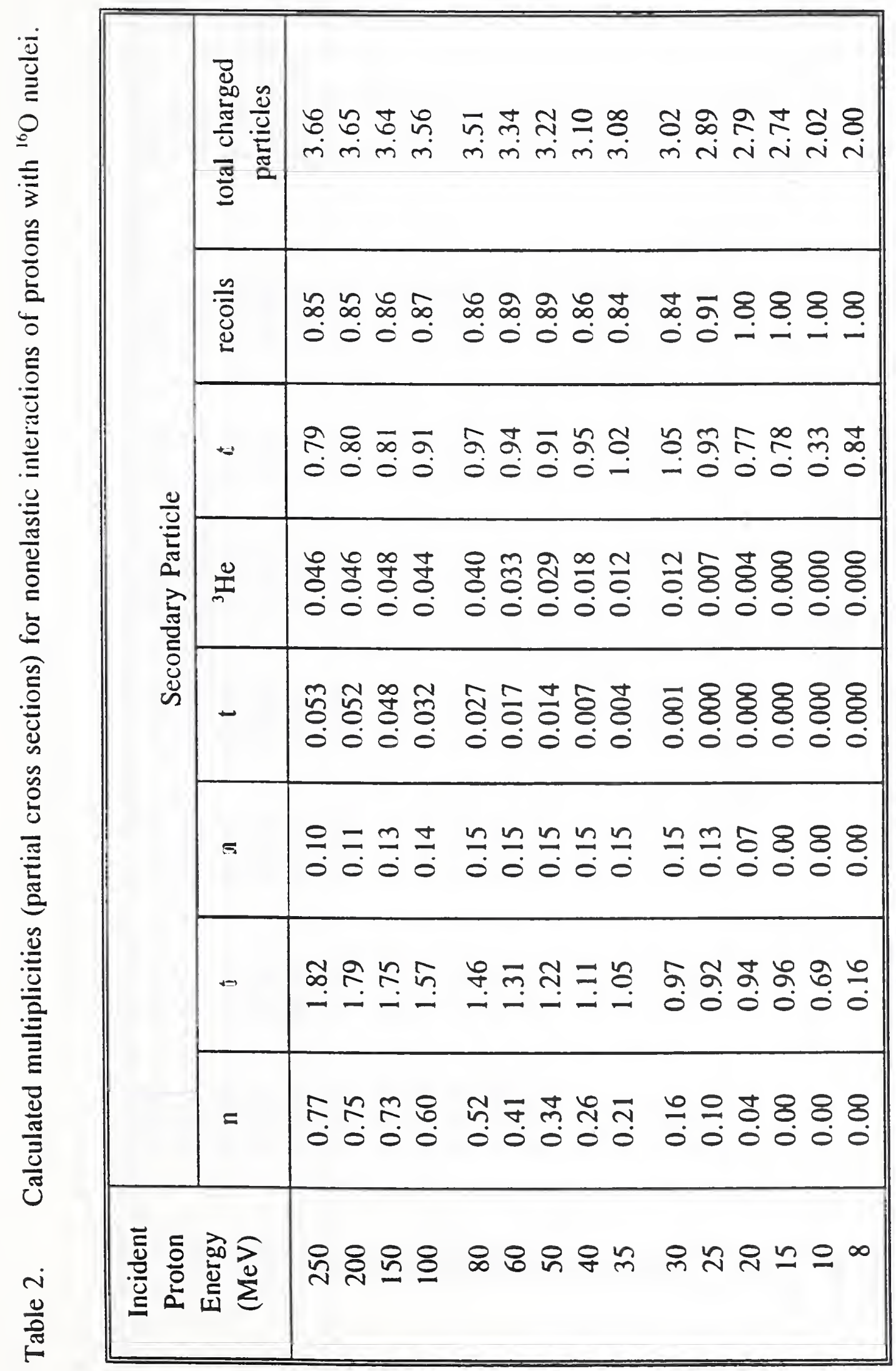




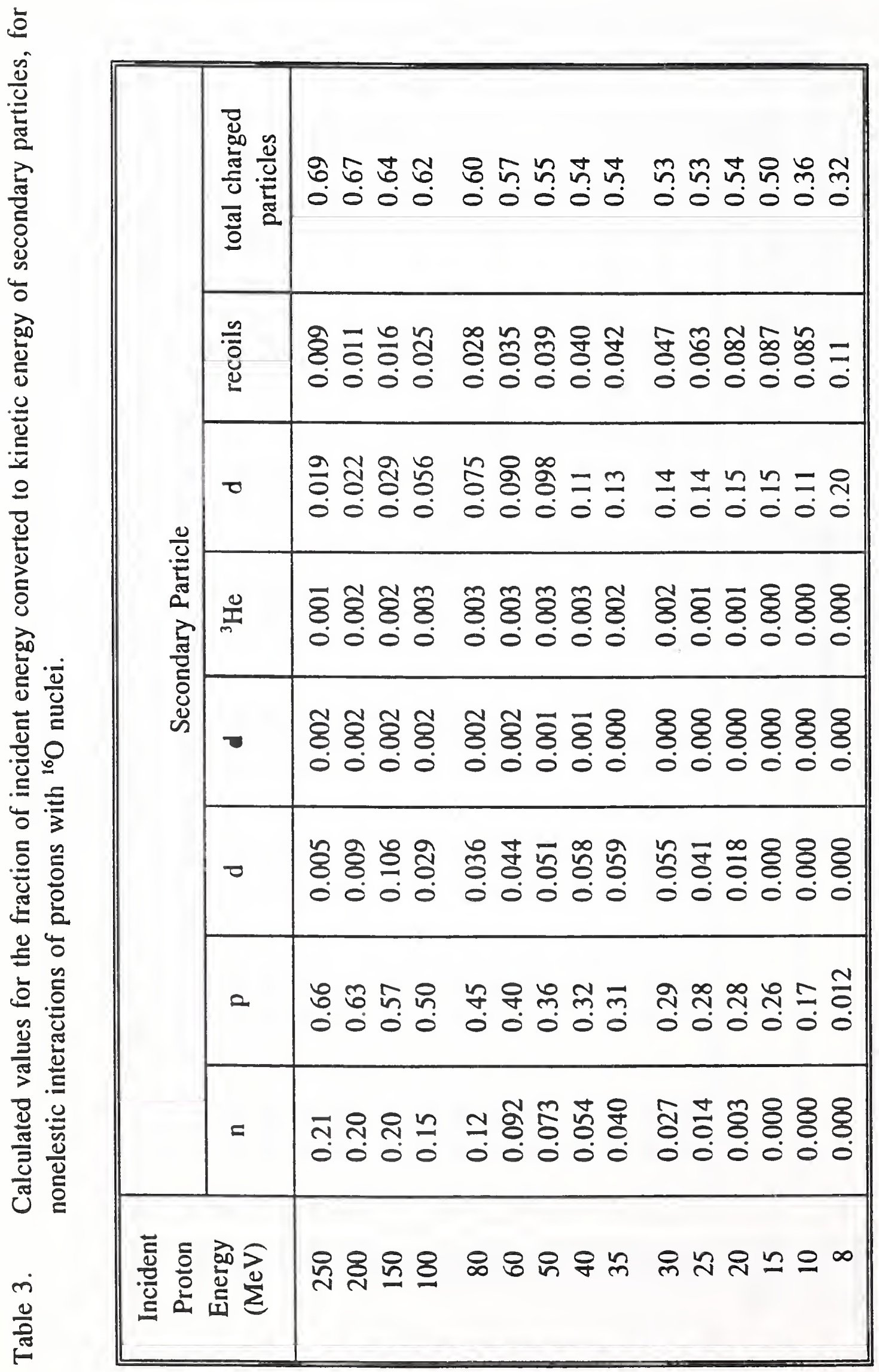




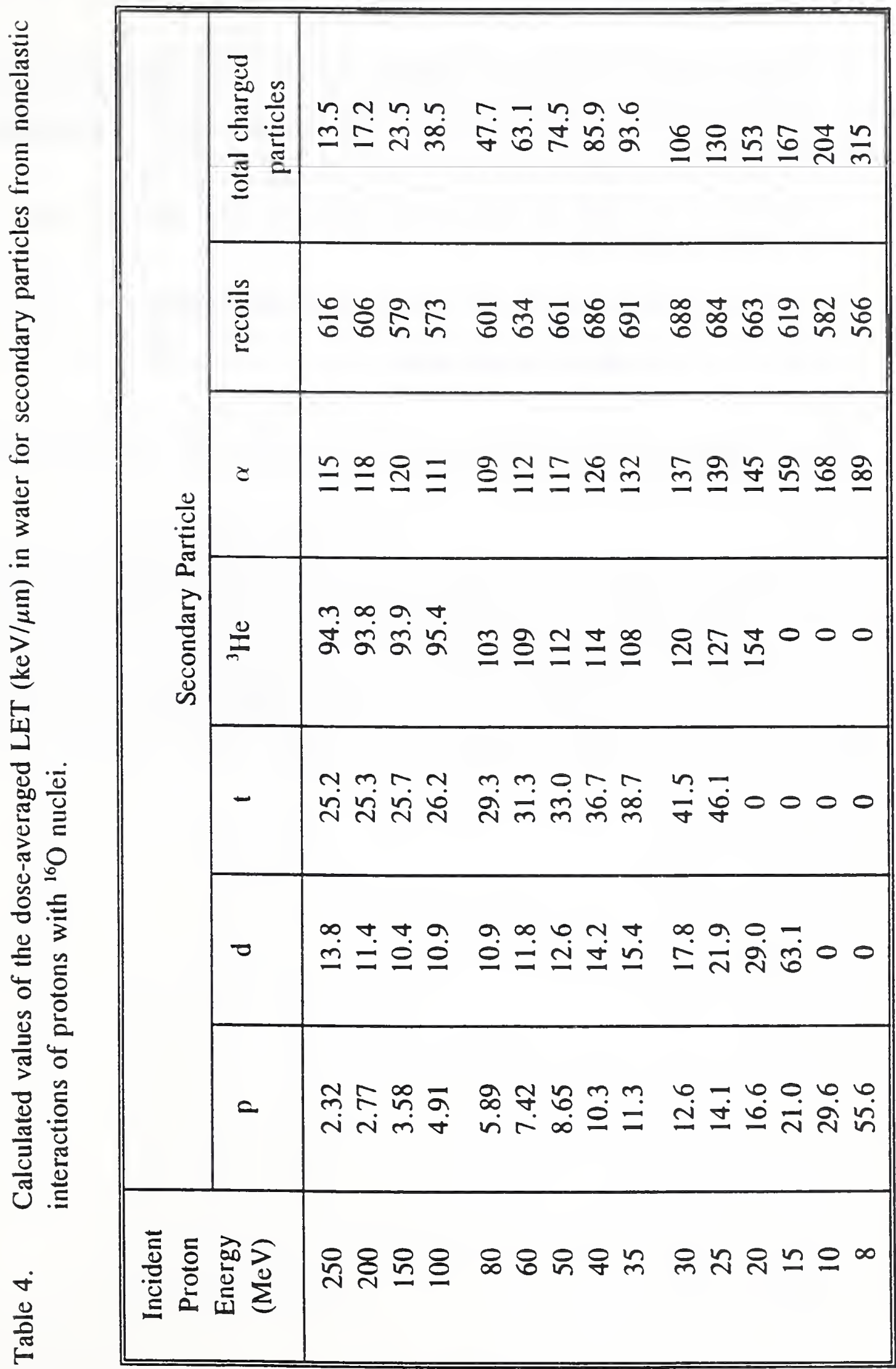


Fig. 1 Total nonelastic nuclear interaction cross sections for $p+{ }^{16} 0$. Experimental results are from Chapman and Macleod (1967) at 13.1 MeV, from Renberg et al. (1972) for 231 to $552 \mathrm{MeV}$, and from Carlson et al. (1975) 18.8 to $47.7 \mathrm{MeV}$; quoted error bars are shown for selected points.

a. Comparison of calculated and experimental values; the solid curve represents the cross section adopted here.

b. Comparison of data from Janni (1982), measured and adopted values.

c. Linear plot of measured and adopted values.

Fig. 2 Fractions of initial number and energy of primary protons lost in nonelastic nuclear interactions during the course of slowing down in water.

Fig. 3 Nuclide-production cross sections for $p+{ }^{16} \mathrm{O}$. Results are given for selected nuclides. The solid curves are from INCA-FBRK calculations, and the dashed curves are from ALICE91 calculations, both normalized to the adopted total nonelastic nuclear interaction cross section. The experimental points are as follows: circles from Moyle et al. (1979); squares from Rayudu (1964); upward triangles from Foley et al. (1962); downward triangles from Yiou et al. (1968); diamonds from Bernas et al. (1965, 1967); five-pointed stars from Valentin et al. (1963); pluses from Epherre et al. (1969); crosses from Albouy et al. (1962); and six-pointed stars from Raisbeck and Yiou (1977).
a. Production of ${ }^{6} \mathrm{Li}$.
b. Production of ${ }^{7} \mathrm{Li}$.
c. Production of ${ }^{7} \mathrm{Be}$.
d. Production of ${ }^{9} \mathrm{Be}$.
e. Production of ${ }^{10} \mathrm{Be}$.
f. Production of ${ }^{10} \mathrm{~B}$.
g. Production of ${ }^{11} \mathrm{~B}$.
h. Production of ${ }^{10} \mathrm{C}$.
i. Production of ${ }^{11} \mathrm{C}$. 
j. Production of ${ }^{13} \mathrm{~N}$.

k. Production of ${ }^{15} \mathrm{O}$.

Fig. 4 Average fraction of the primary proton kinetic energy converted to kinetic energy of all secondary charged particles in nonelastic interactions $\mathrm{p}+{ }^{16} \mathrm{O}$. The points represent results from INCA-FBRK calculations; the curve is fitted. This fraction is assumed absorbed locally.

Fig. 5 Spectra, integrated over angle, of secondary particles produced in nonelastic nuclear interactions of $\mathrm{p}+{ }^{16} \mathrm{O}$ for incident proton energies of $250 \mathrm{MeV}$ and below. The spectra are normalized to one emitted secondary particle.

a. Secondary neutrons.

b. Secondary protons.

c. Secondary deuterons.

d. Secondary tritons.

e. Secondary ${ }^{3} \mathrm{He}$.

f. Secondary $\alpha$ particles.

g. Mass 6 recoils.

h. Mass 7 recoils.

i. Mass 8 recoils

j. Mass 9 recoils

k. Mass 10 recoils.

1. Mass 11 recoils.

m. Mass 12 recoils.

n. Mass 13 recoils.

o. Mass 14 recoils.

p. Mass 15 recoils. 
q. Mass 16 recoils.

Fig. 6 Stopping powers of water for nuclear secondary particle from $p+{ }^{16} O$. The results, given for particles and ions with atomic numbers $Z$ from 1 to 8 and mass numbers from 1 to 16 , are plotted as a function of particle energy in units of the atomic mass, so that curves for particles of the same $Z$ nearly coincide. The curves separate at low energies to show both the total stopping powers (upper curves) and the collision stopping powers (lower curves).

Fig. 7 Differential tracklength distributions for secondary charged particles from $100-\mathrm{MeV}$ proton nonelastic nuclear interactions slowing down in water. The results are normalized to one nuclear interaction by the primary proton.
a. Secondary protons.
b. Secondary deuterons.
c. Secondary tritons.
d. Secondary ${ }^{3} \mathrm{He}$.
e. Secondary $\alpha$ particles.
f. Mass 6 recoils.
g. Mass 7 recoils.
h. Mass 8 recoils
i. Mass 9 recoils
j. Mass 10 recoils.
k. Mass 11 recoils.
1. Mass 12 recoils.
m. Mass 13 recoils.
n. Mass 14 recoils.
o. Mass 15 recoils.
p. Mass 16 recoils. 
Fig. 8 Dose-averaged LET from the total charged-particle contribution of the nuclear secondaries. Results are given as a function of the energy of the primary proton. The points are from the distributions calculated with INCA-FBRK, and the curve is fitted.

Fig. 9 Dose-averaged LET as a function of depth in a water phantom irradiated by proton beams incident perpendicularly. Results are given for incident unmodulated (monoenergetic) beams and for beams assumed modulated by 21-ridge (water-equivalent) filters to spread out the Bragg peak. Relative depth-dose curves are given in the lower graphs. The doseaveraged LET is given in the upper graphs for the primary-protons-only (dashed curves) and for the primaries-plus-nuclear-secondaries (solid curves).

a. Beam energy $250 \mathrm{MeV}$, modulation range $20 \mathrm{~cm}$.

b. Beam energy $160 \mathrm{MeV}$, modulation range $10 \mathrm{~cm}$.

c. Beam energy $70 \mathrm{MeV}$, modulation range $2.5 \mathrm{~cm}$.

Fig.10 Relative biological effectiveness (RBE) of heavy charged particles, as a function of their associated unrestricted linear energy transfer (LET) in water. The points represent reported RBE values from charged-particle irradiation of mammalian cell systems, and include data from Powers-Riesius (in Shavers et al., 1993), Barendsen (1972), Matsubara et al. (1990), Bettega et al. (1979), Raju et al. (1978), and Courdi et al. (1992). The dashed curves are from simple functions described in the text.

Fig.11 RBE values averaged over all charged-particle secondaries from $\mathrm{p}+{ }^{16} \mathrm{O}$ nuclear reactions slowing down in water. The points calculated from the data illustrated in Figs. 7 and 10 are fitted by the solid curves. The "high" and "low" designations correspond to use of the upper and lower curves in Fig. 10.

Fig.12 Calculated RBE values as a function of depth in a water phantom irradiated by proton beams incident perpendicularly. Results are given for incident unmodulated beams (dashed curves) and for modulated beams (solid curves) assuming 21-ridge (water-equivalent) filters to spread out the Bragg peak. Relative depth-dose curves are given in the lower graphs. The average RBE is given in the upper graphs for the assumed "high" and "low" responses in Figs. 10 and 11.

a. Beam energy $250 \mathrm{MeV}$, modulation range $20 \mathrm{~cm}$.

b. Beam energy $160 \mathrm{MeV}$, modulation range $10 \mathrm{~cm}$.

c. Beam energy $70 \mathrm{MeV}$, modulation range $2.5 \mathrm{~cm}$. 
Fig.13 Expanded plot of calculated RBE values in the distal region of the Bragg peak for $160-\mathrm{MeV}$ beams in a water phantom (see caption for Fig 12b). The points represent values measured by Robertson et al. (1975): open circles for the unmodulated beam, closed circles for the modulated beam. 


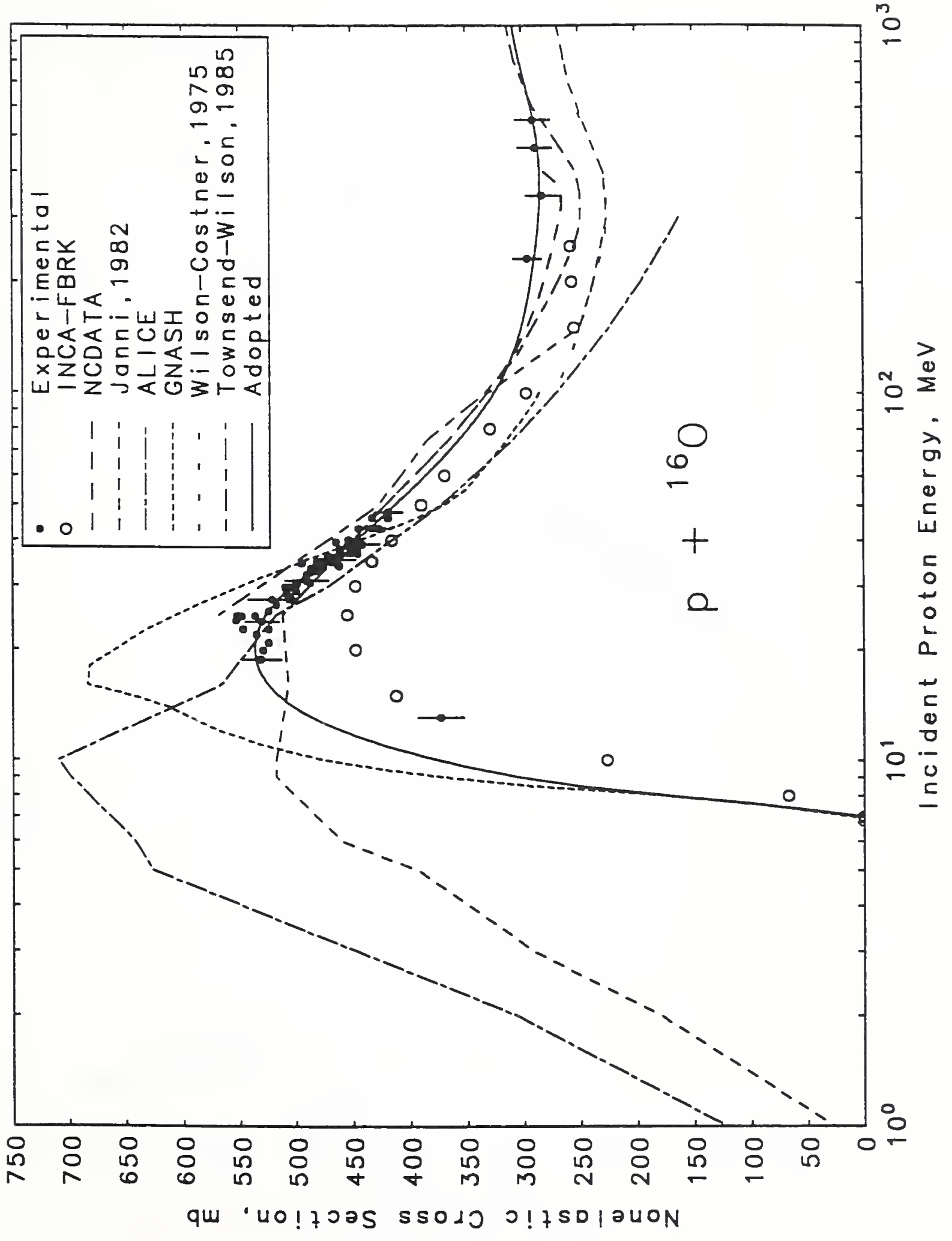




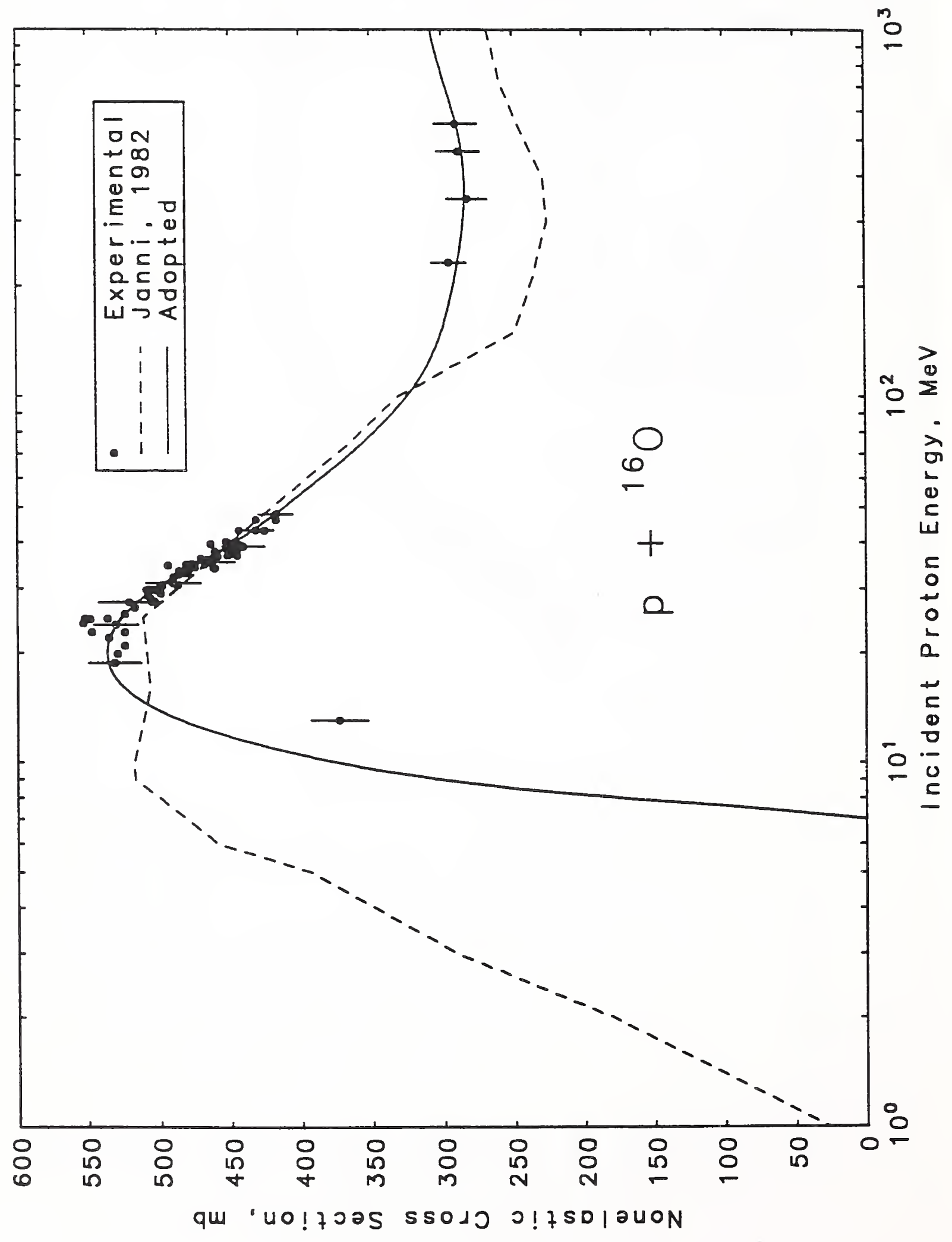




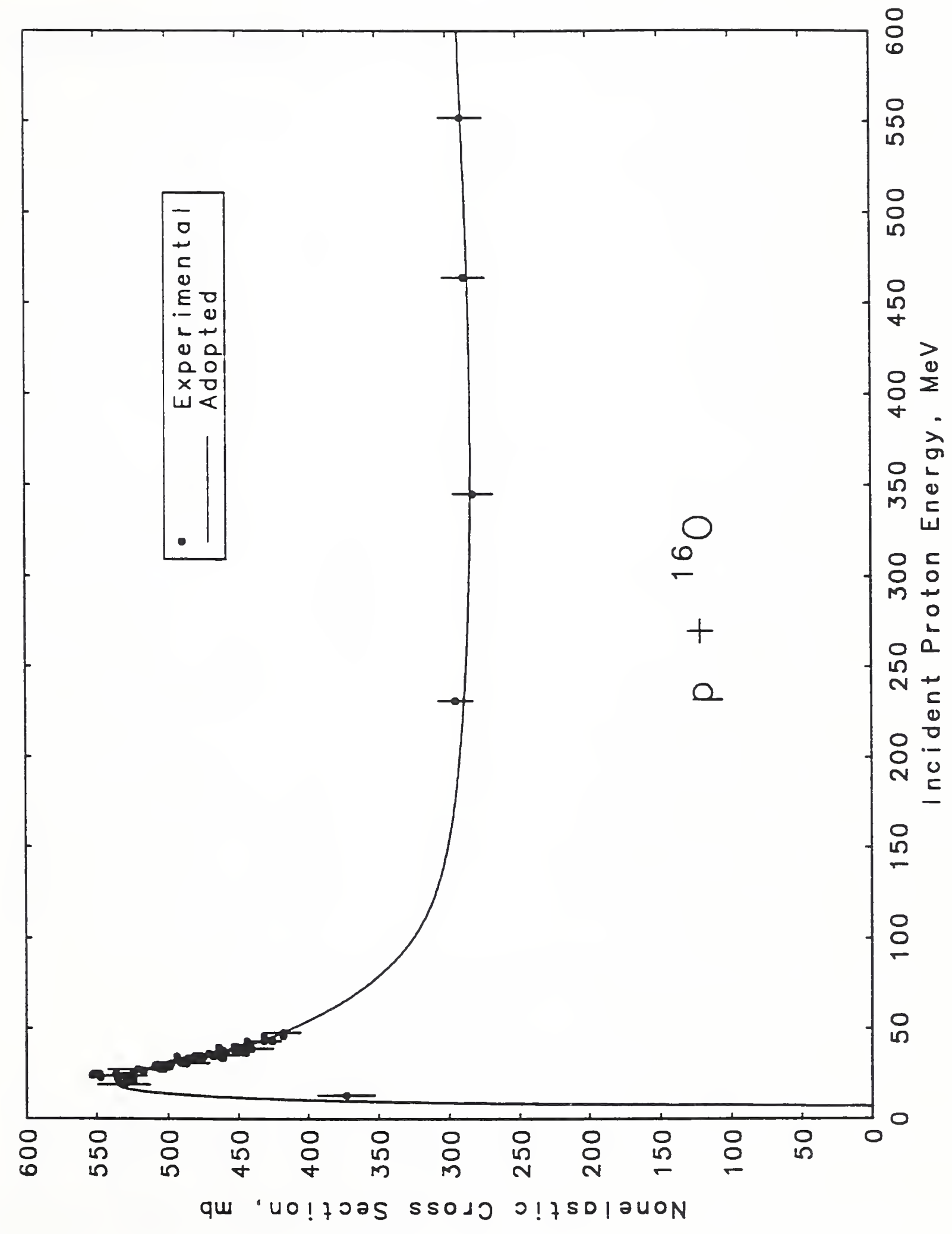




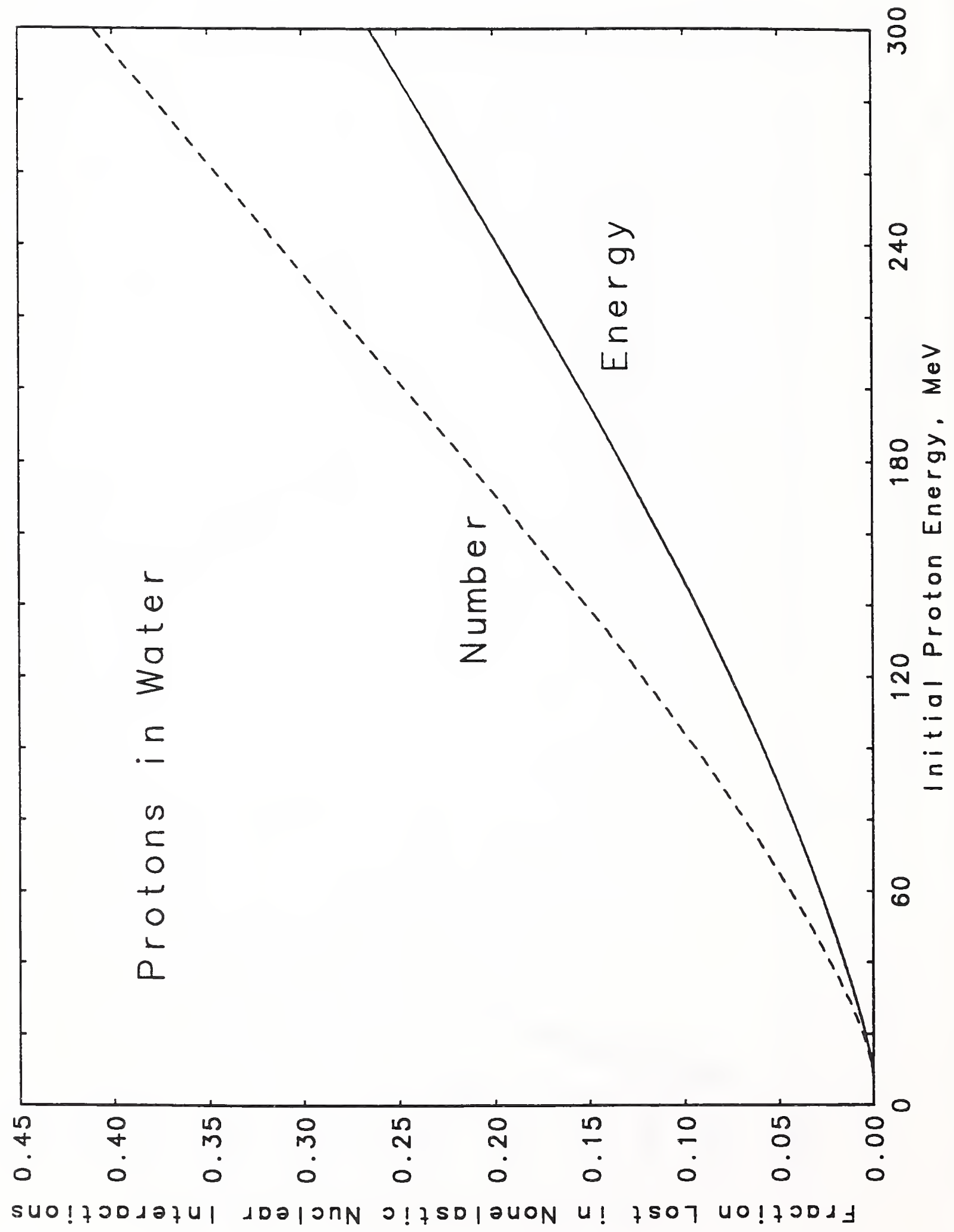




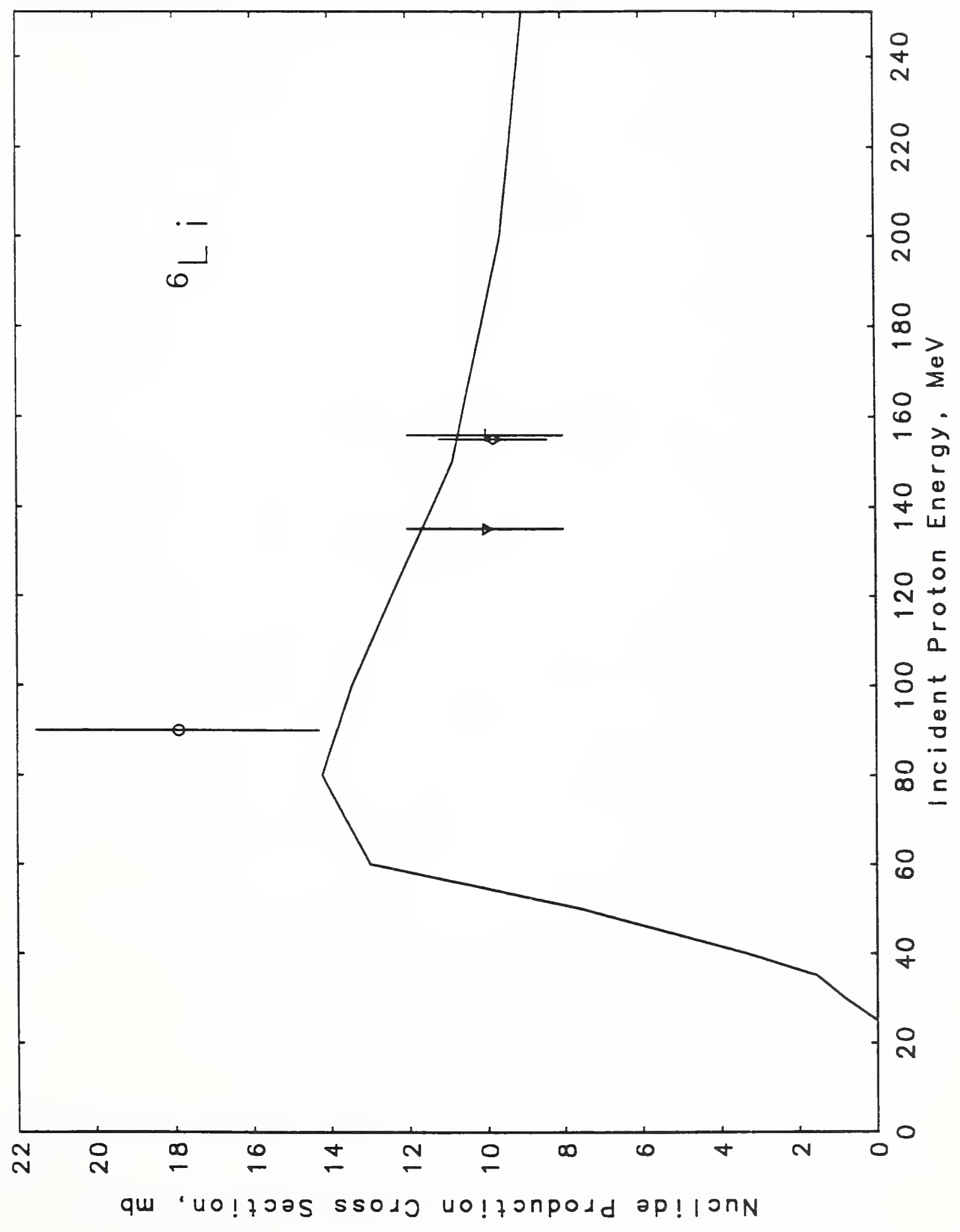




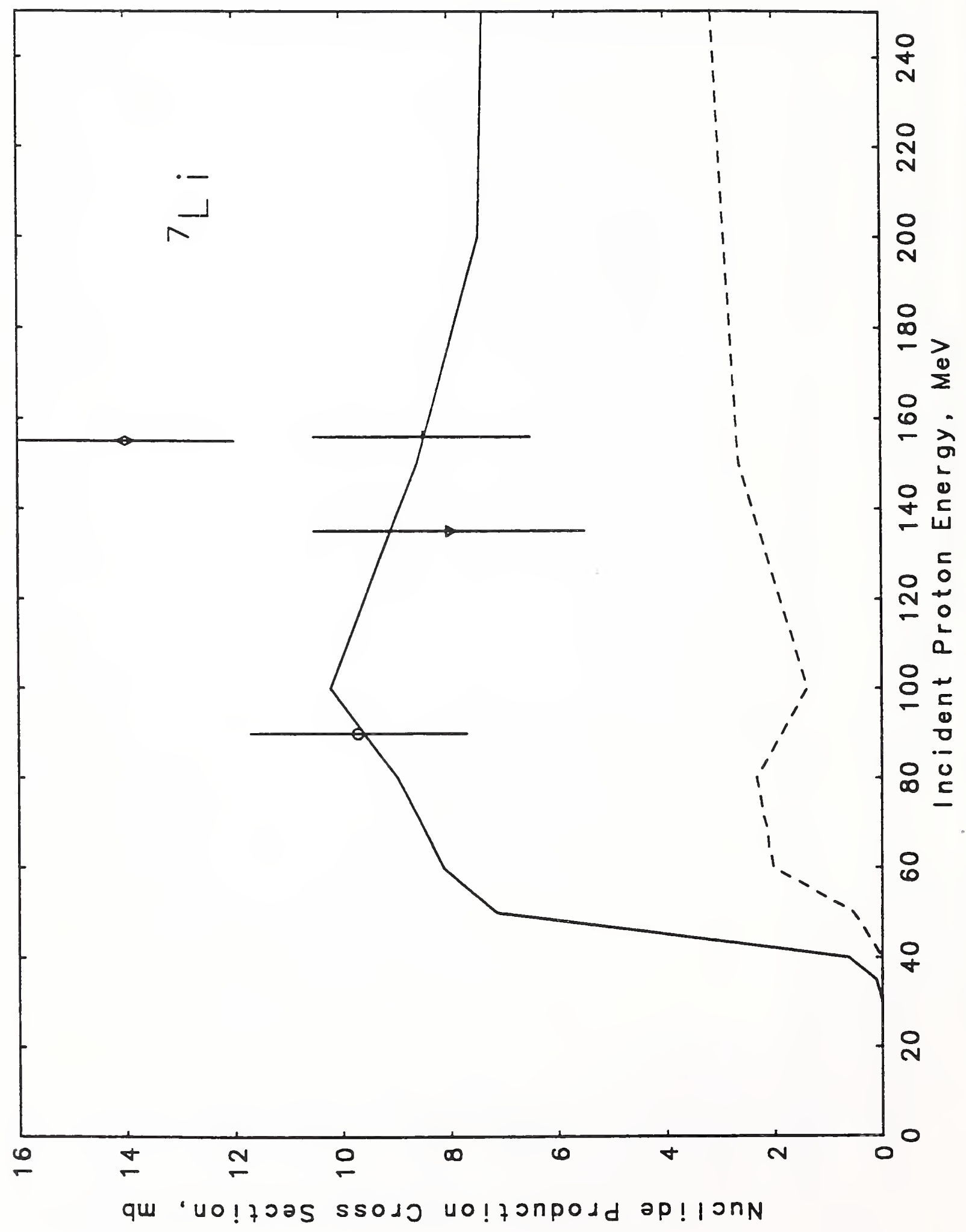

$\frac{\dot{2}}{\dot{m}}$ 


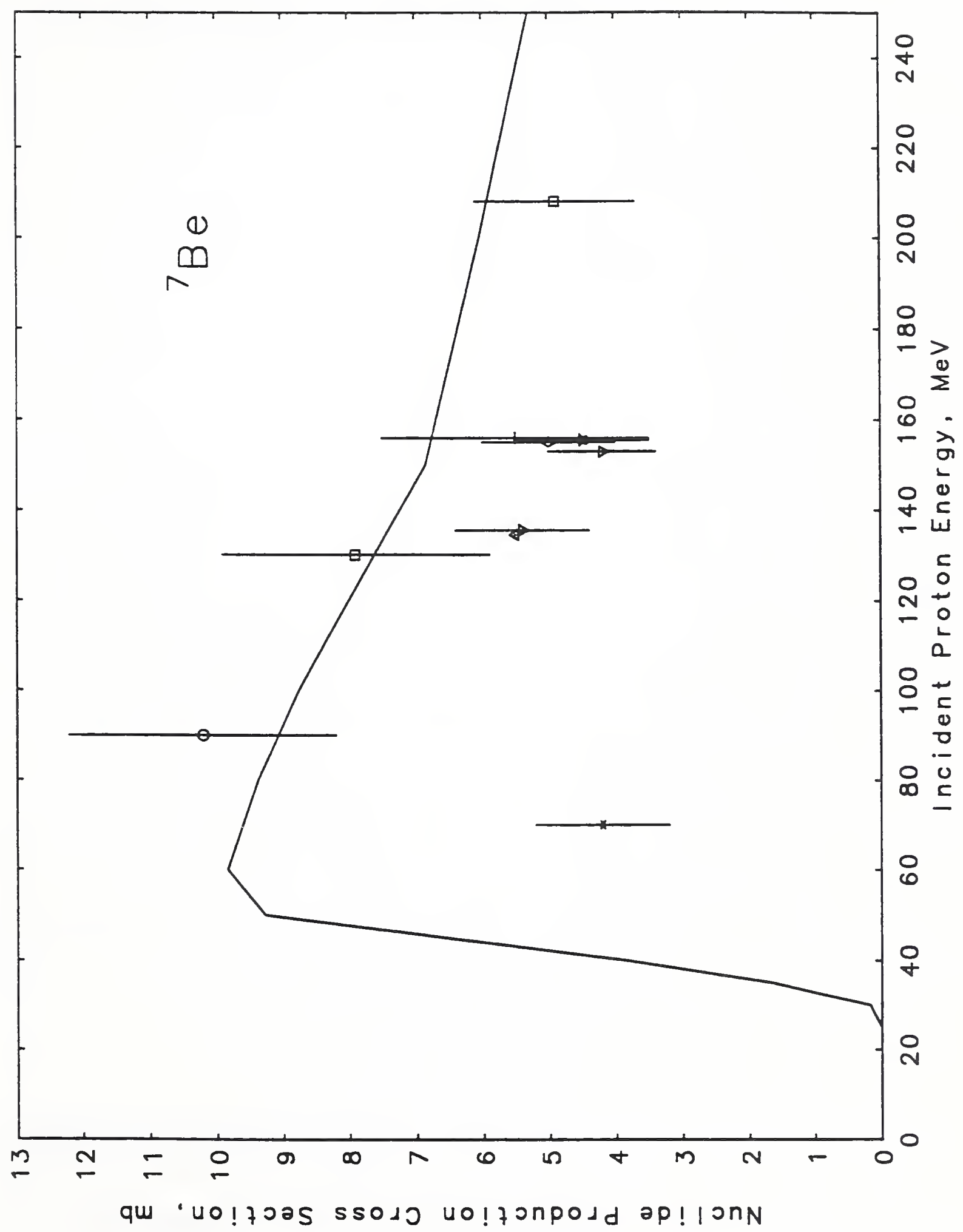




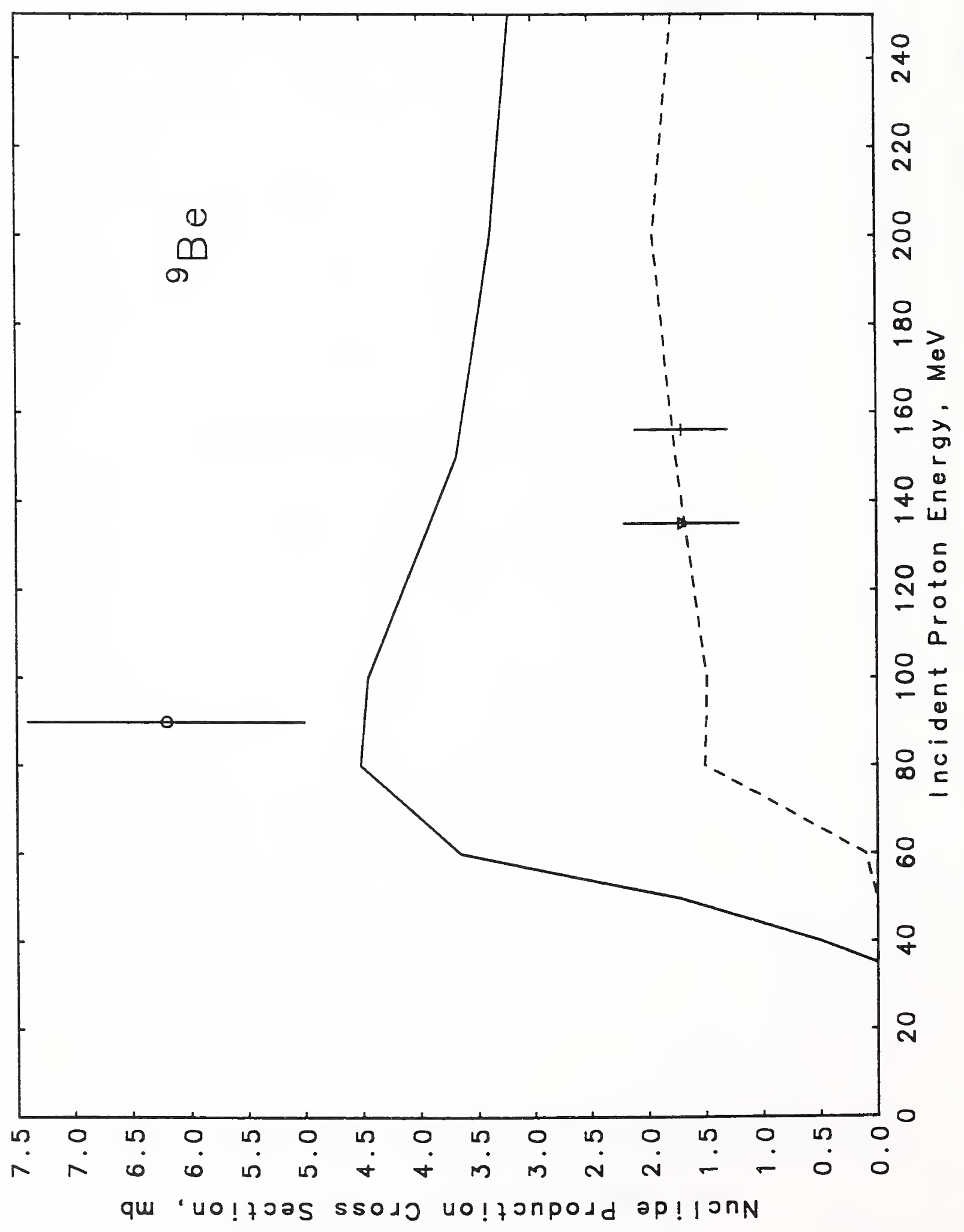




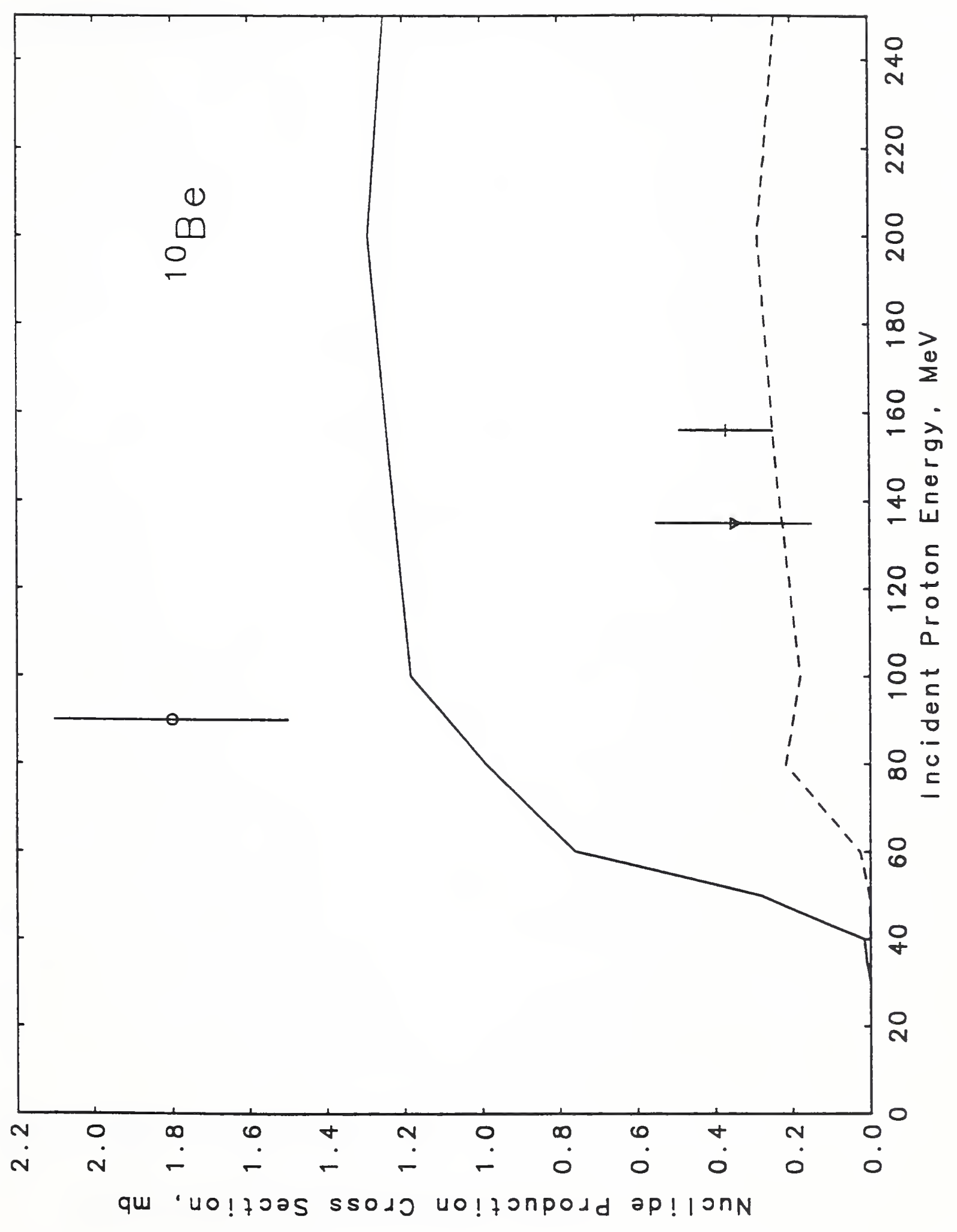




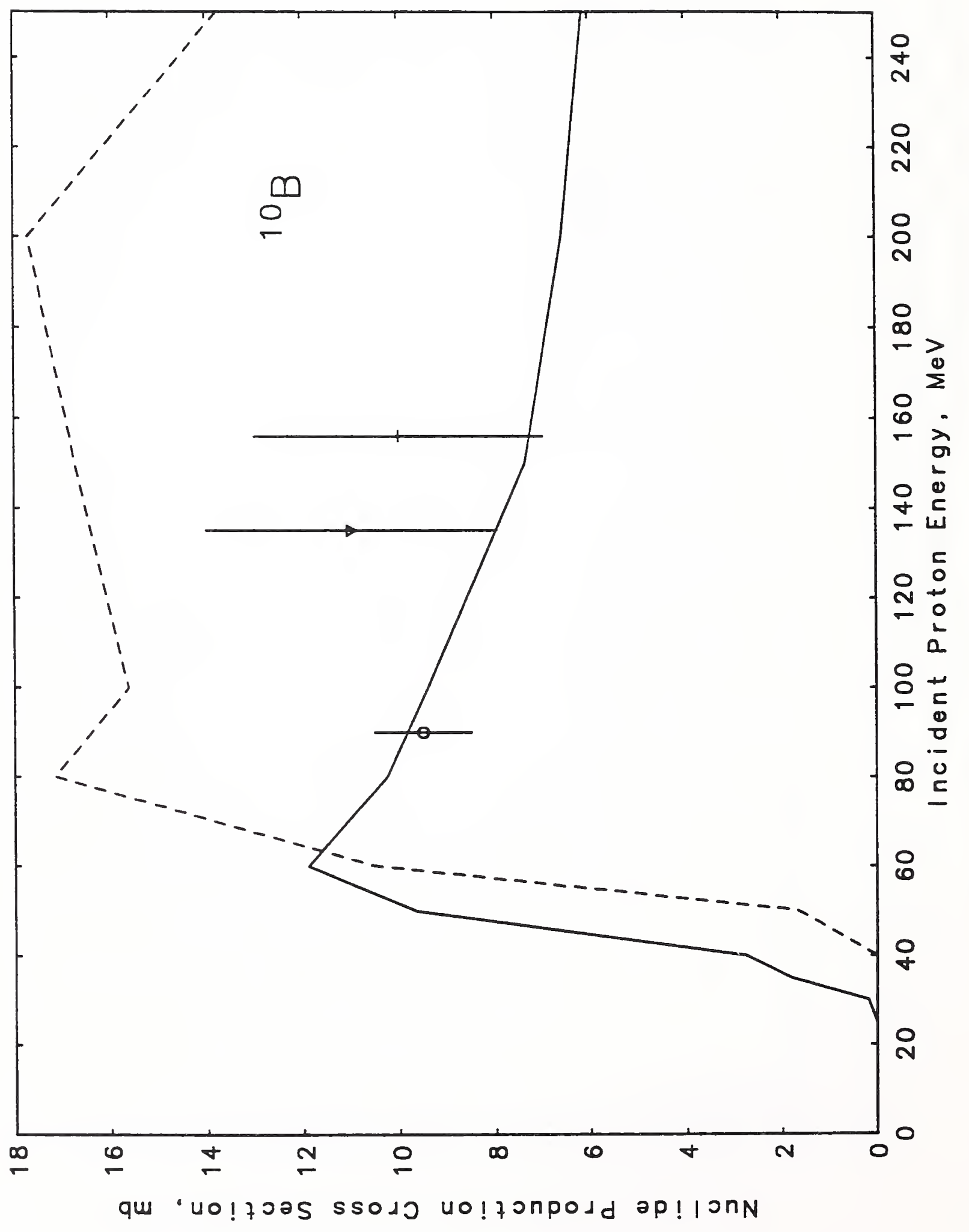

$\stackrel{\dot{m}}{m}$ 


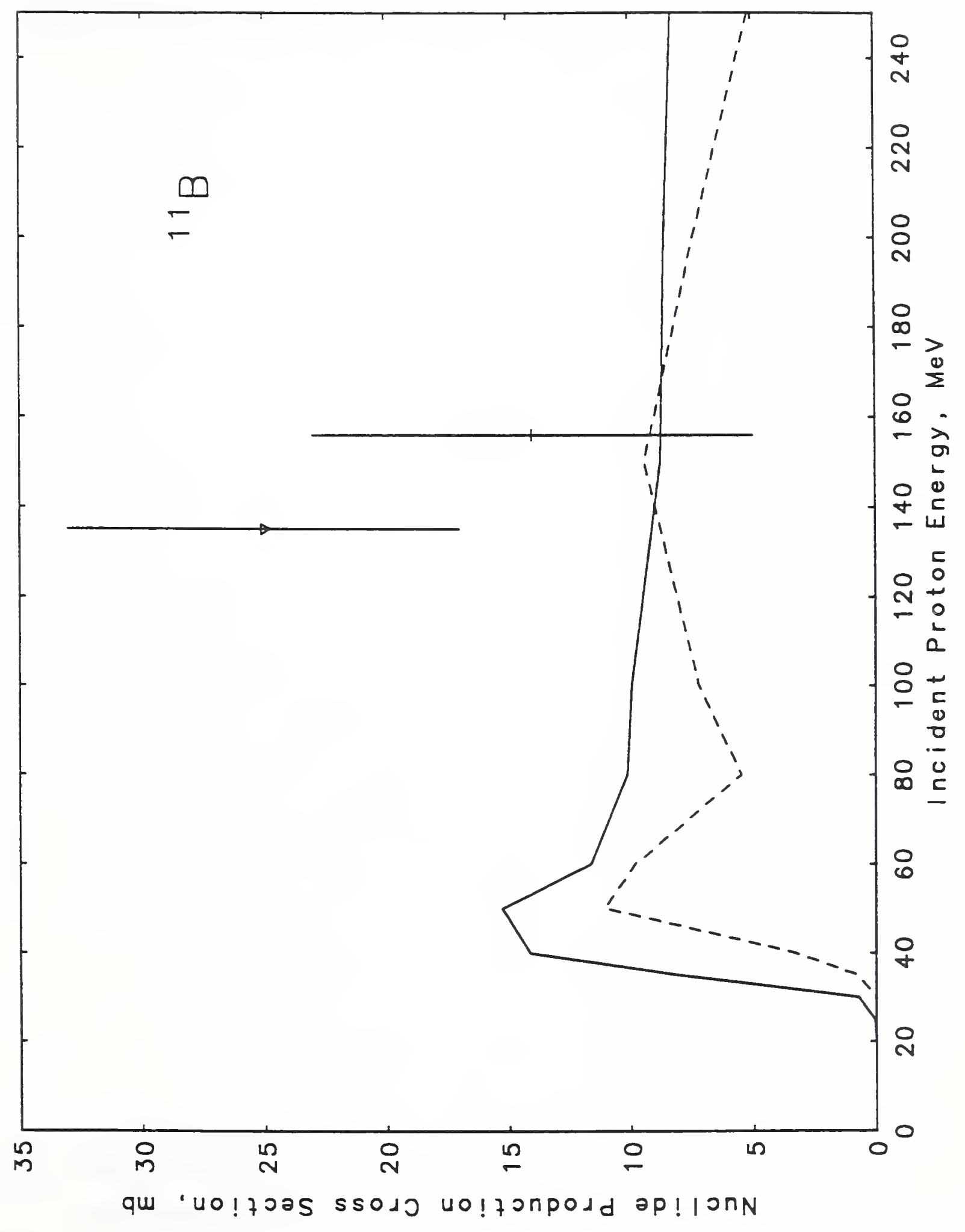




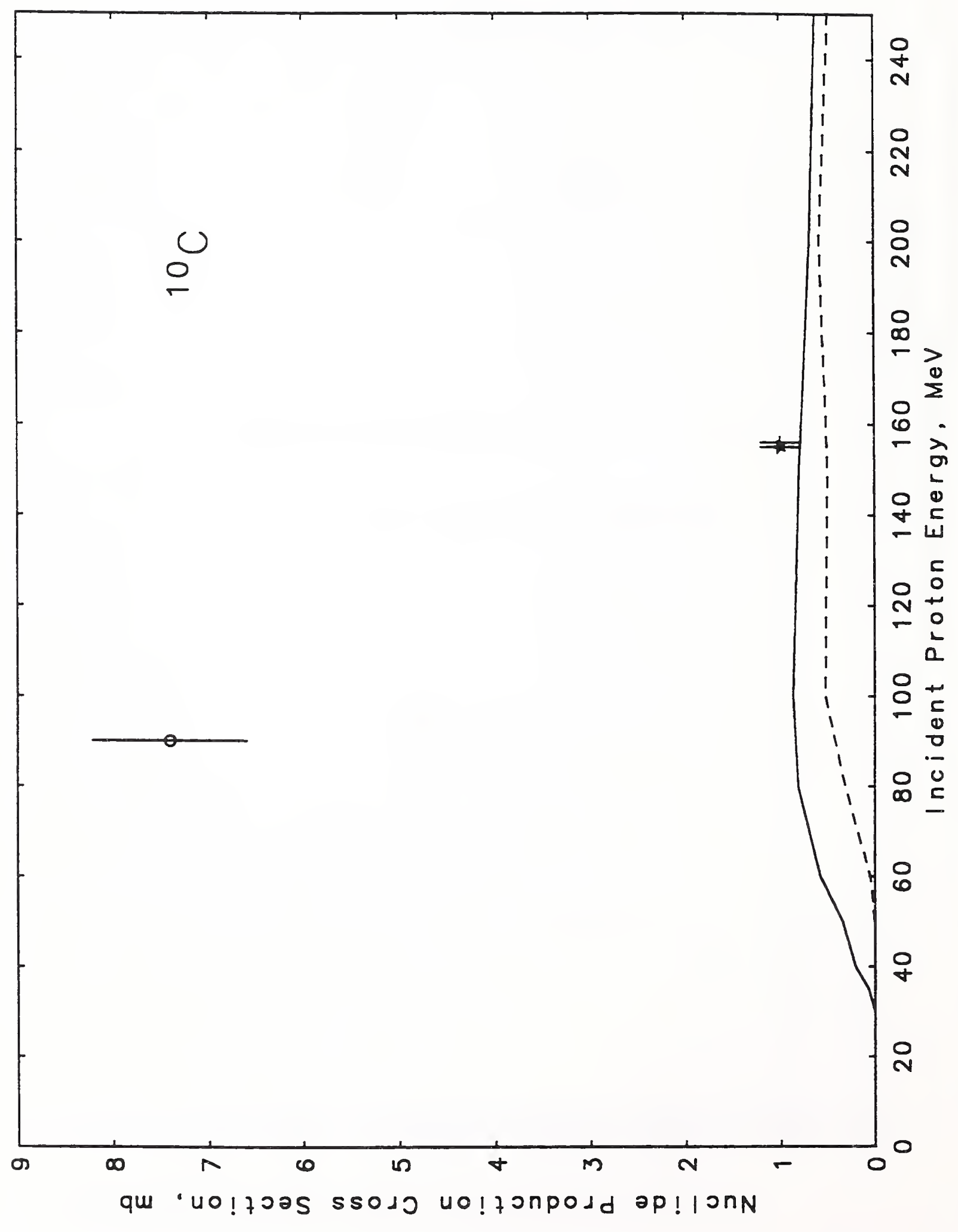




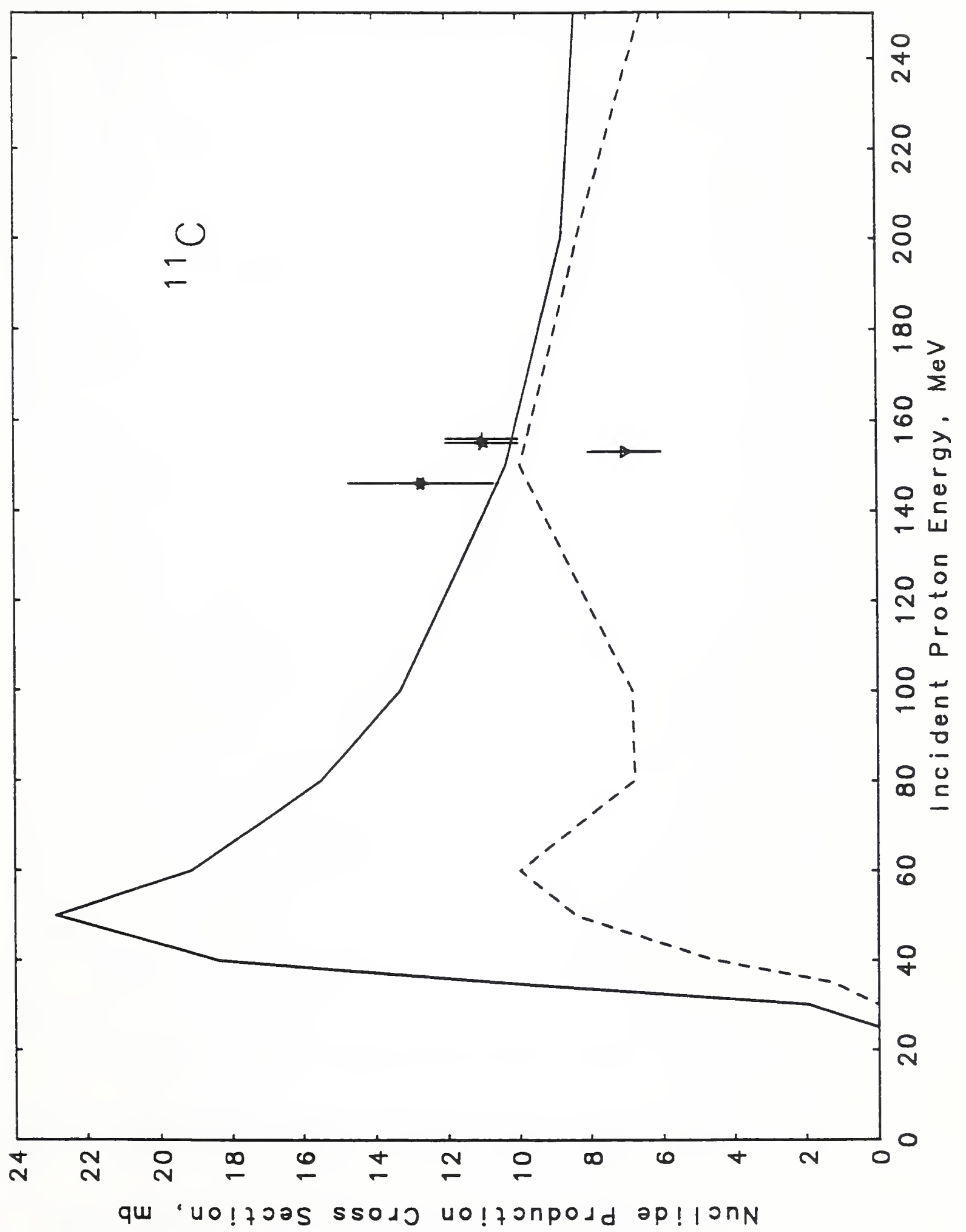




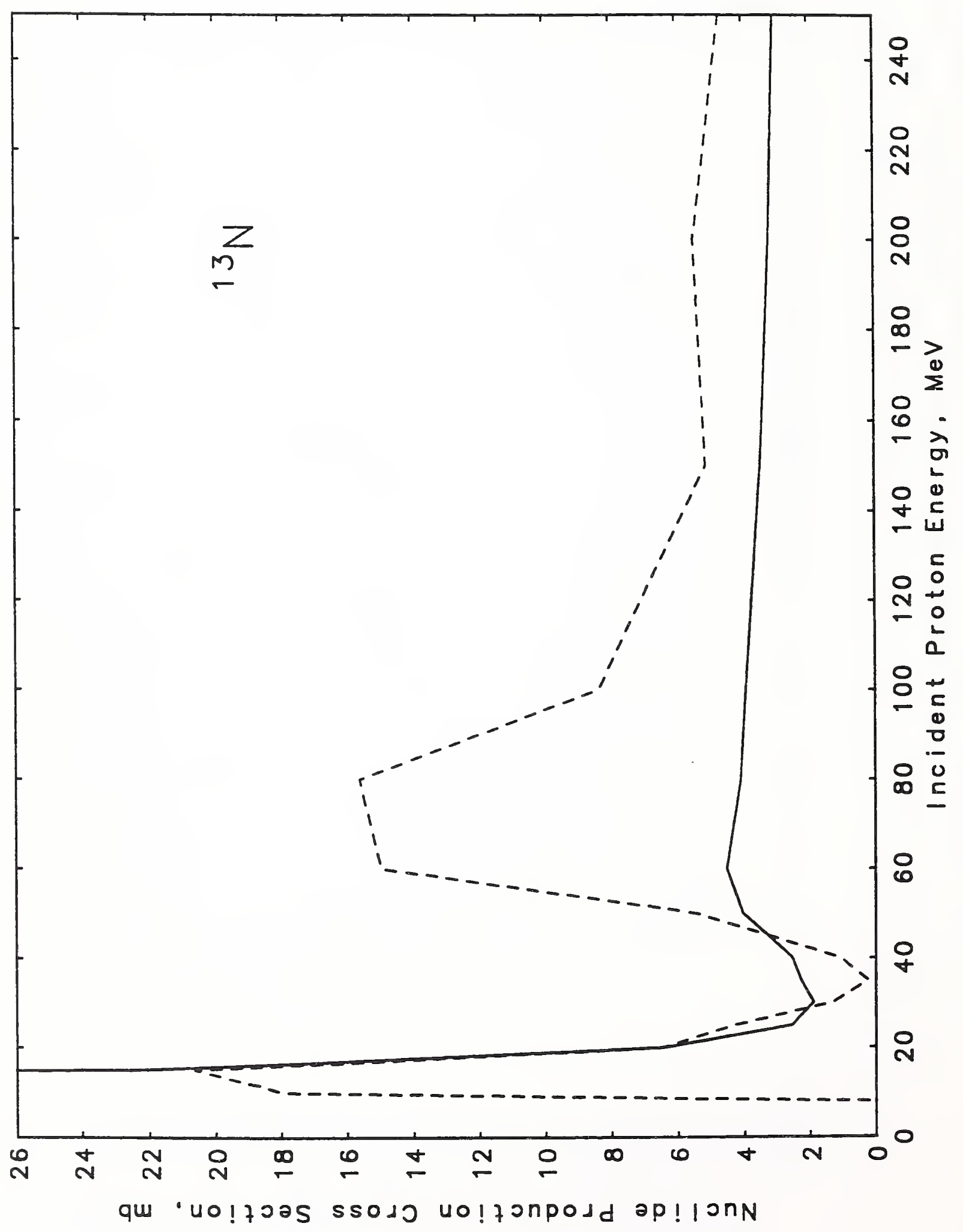




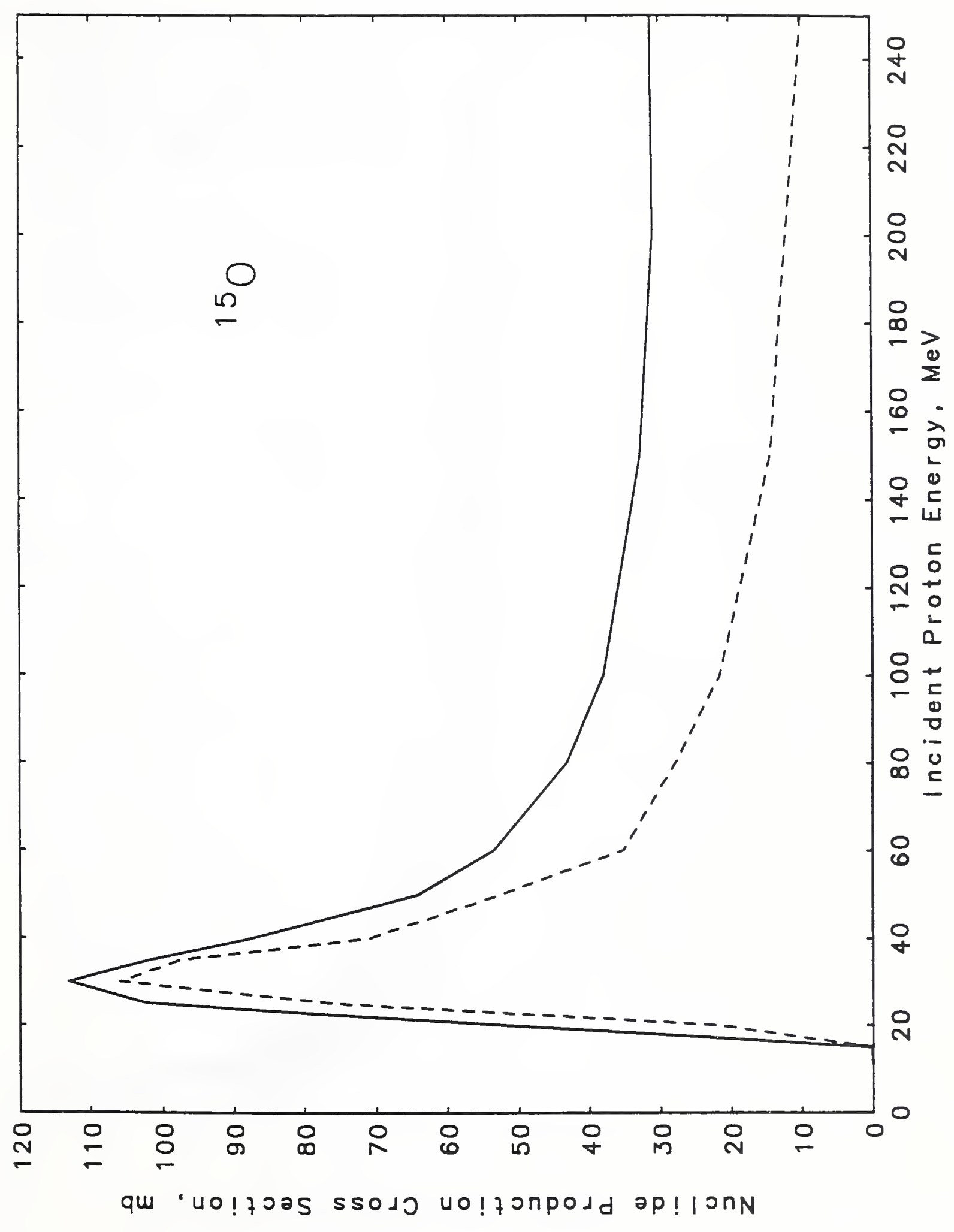




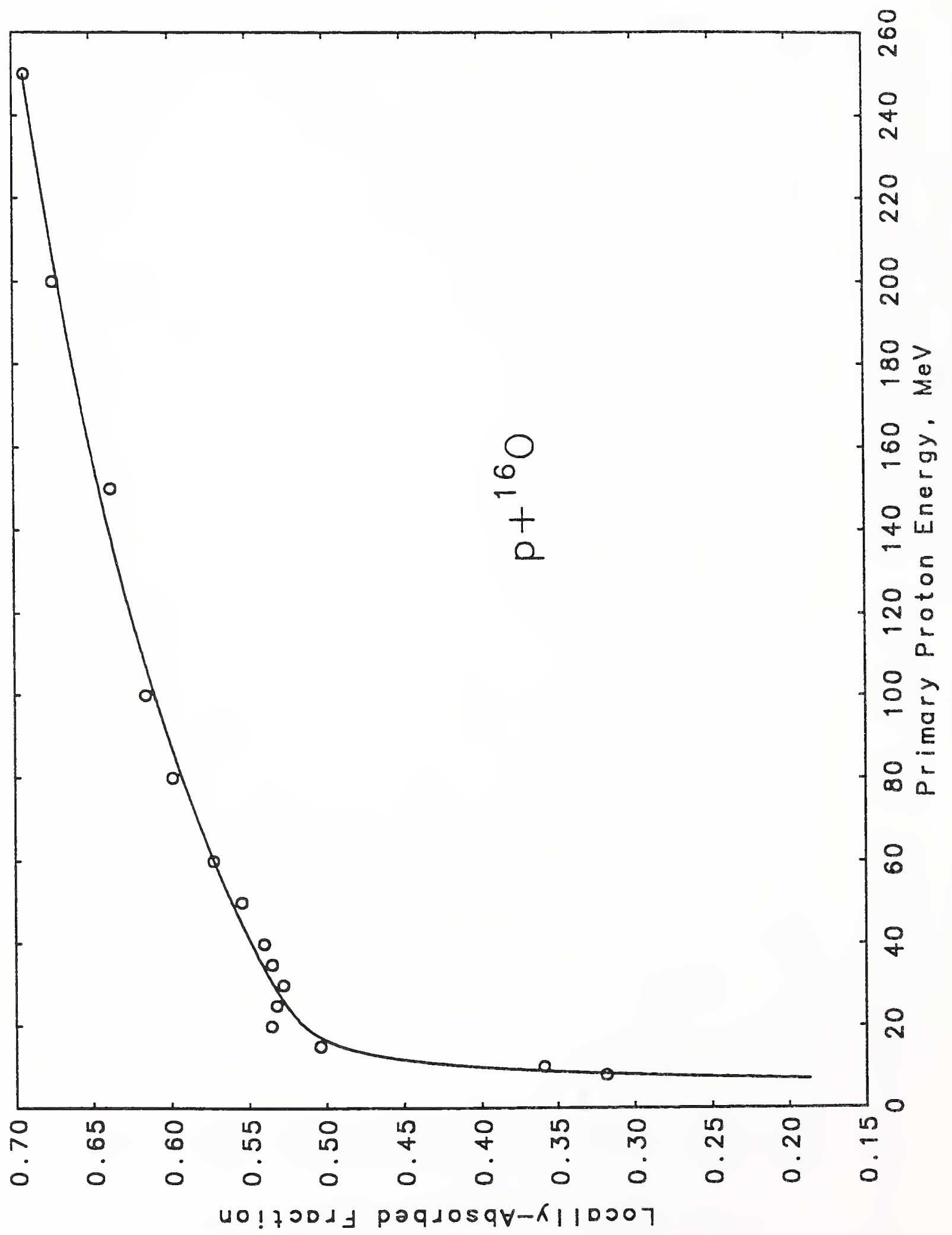




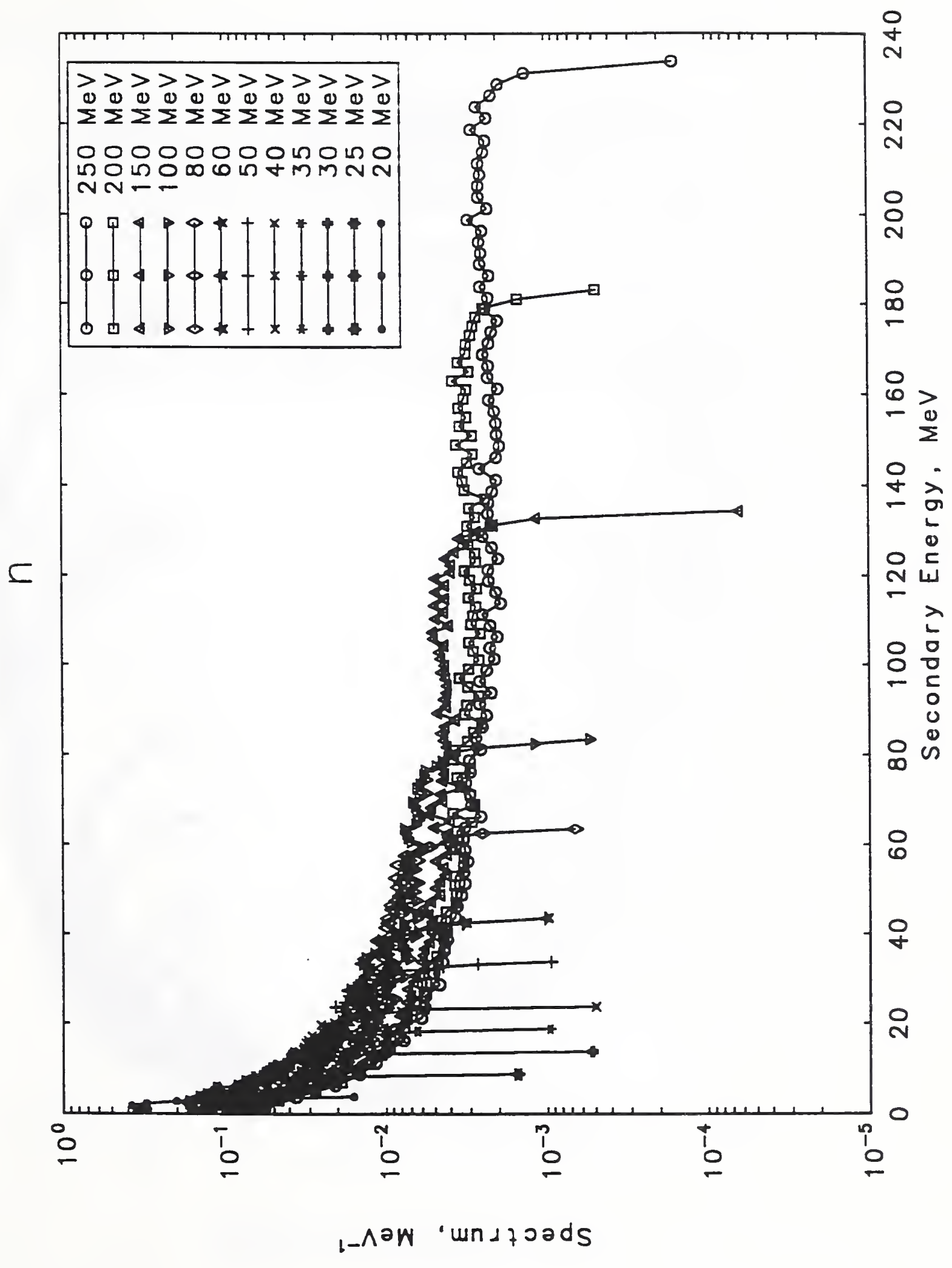




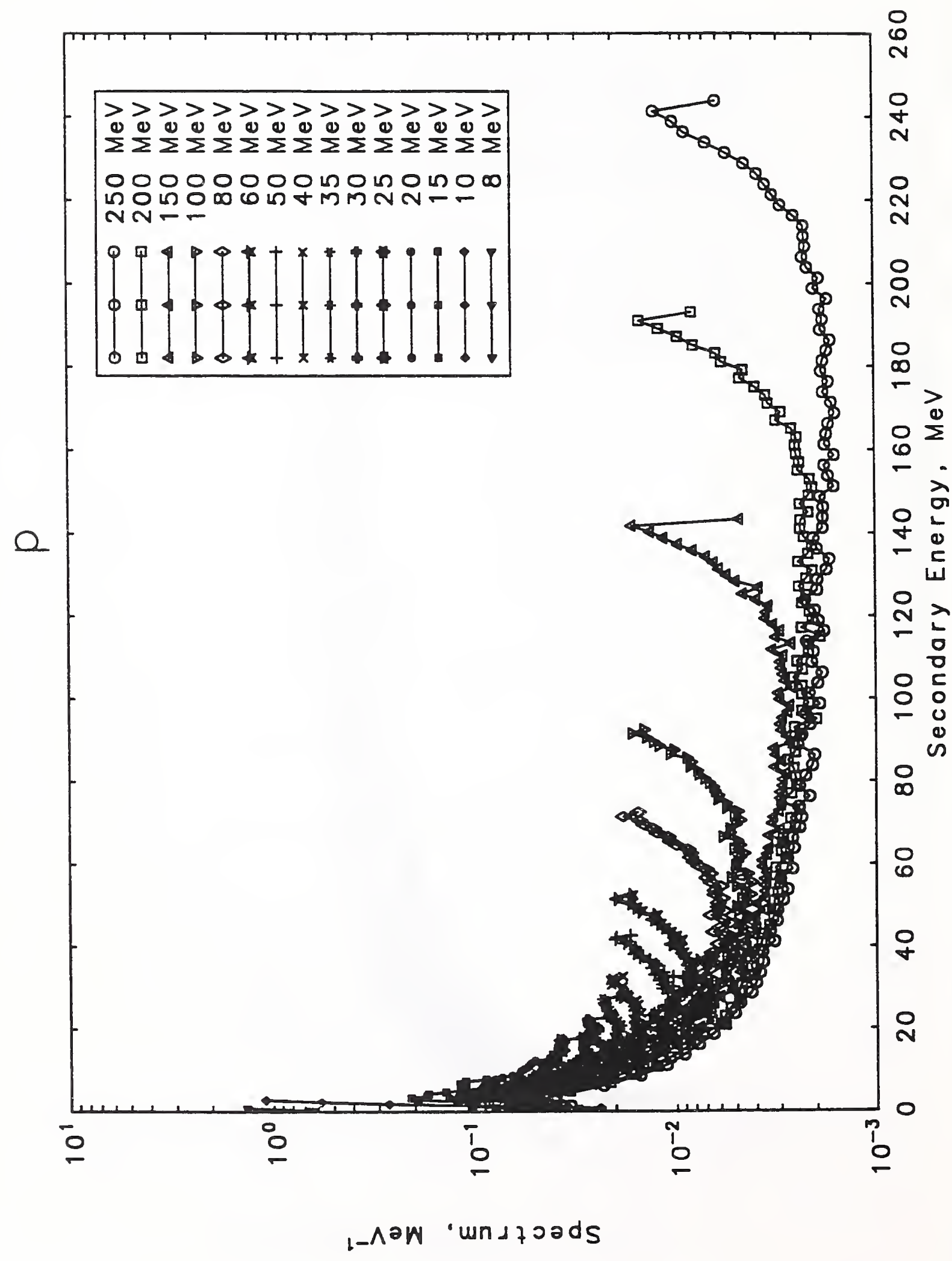

$\frac{2}{n}$ 


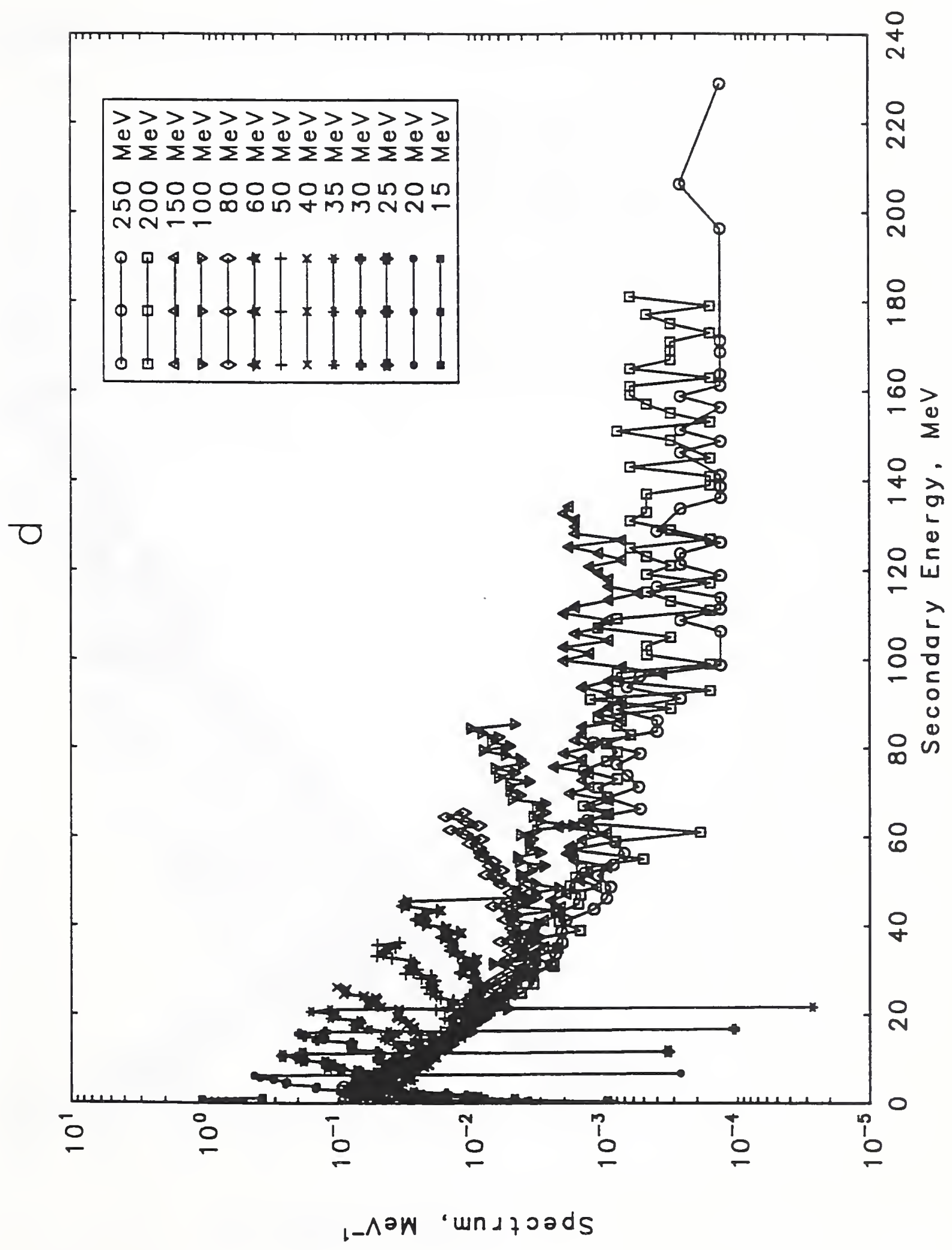




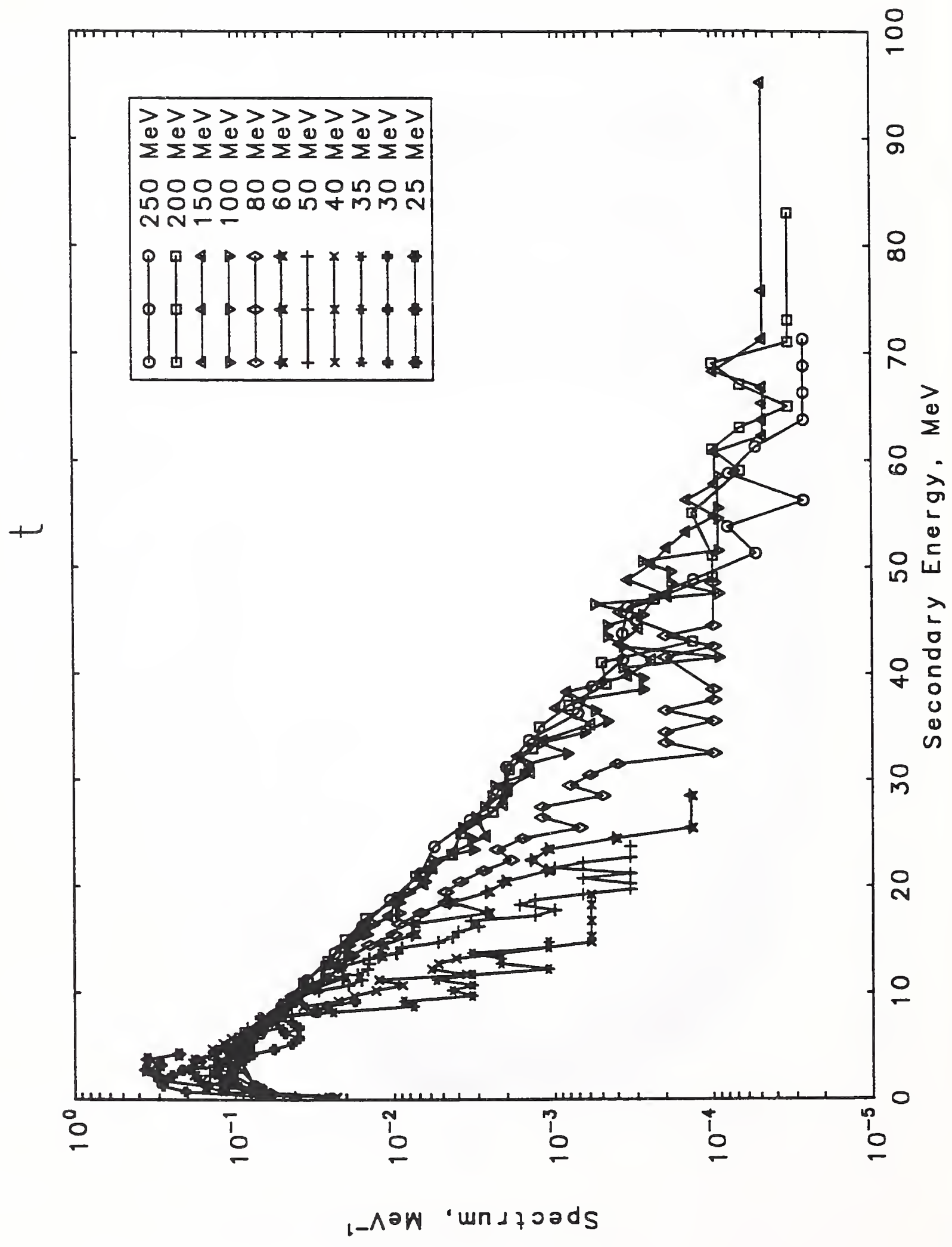




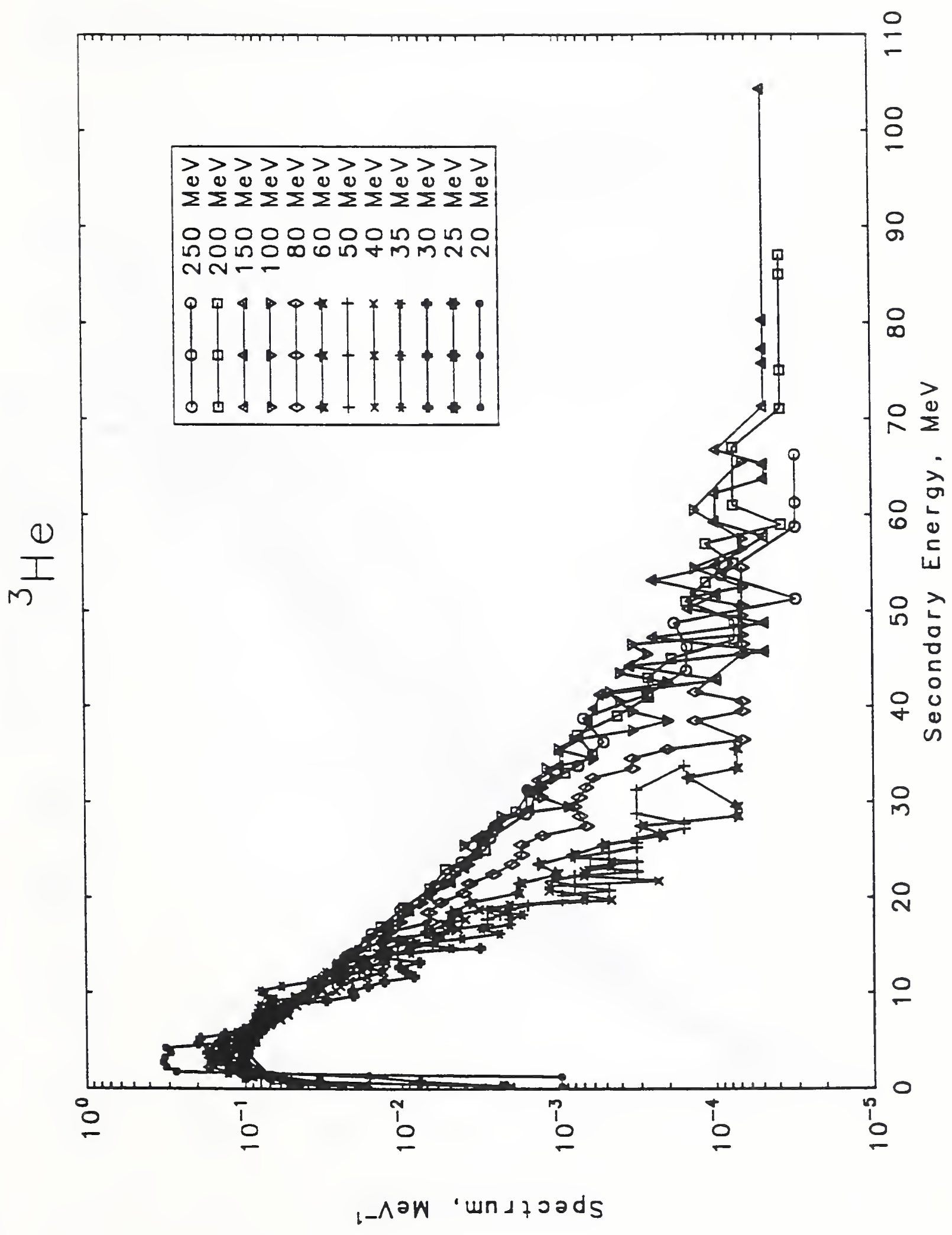




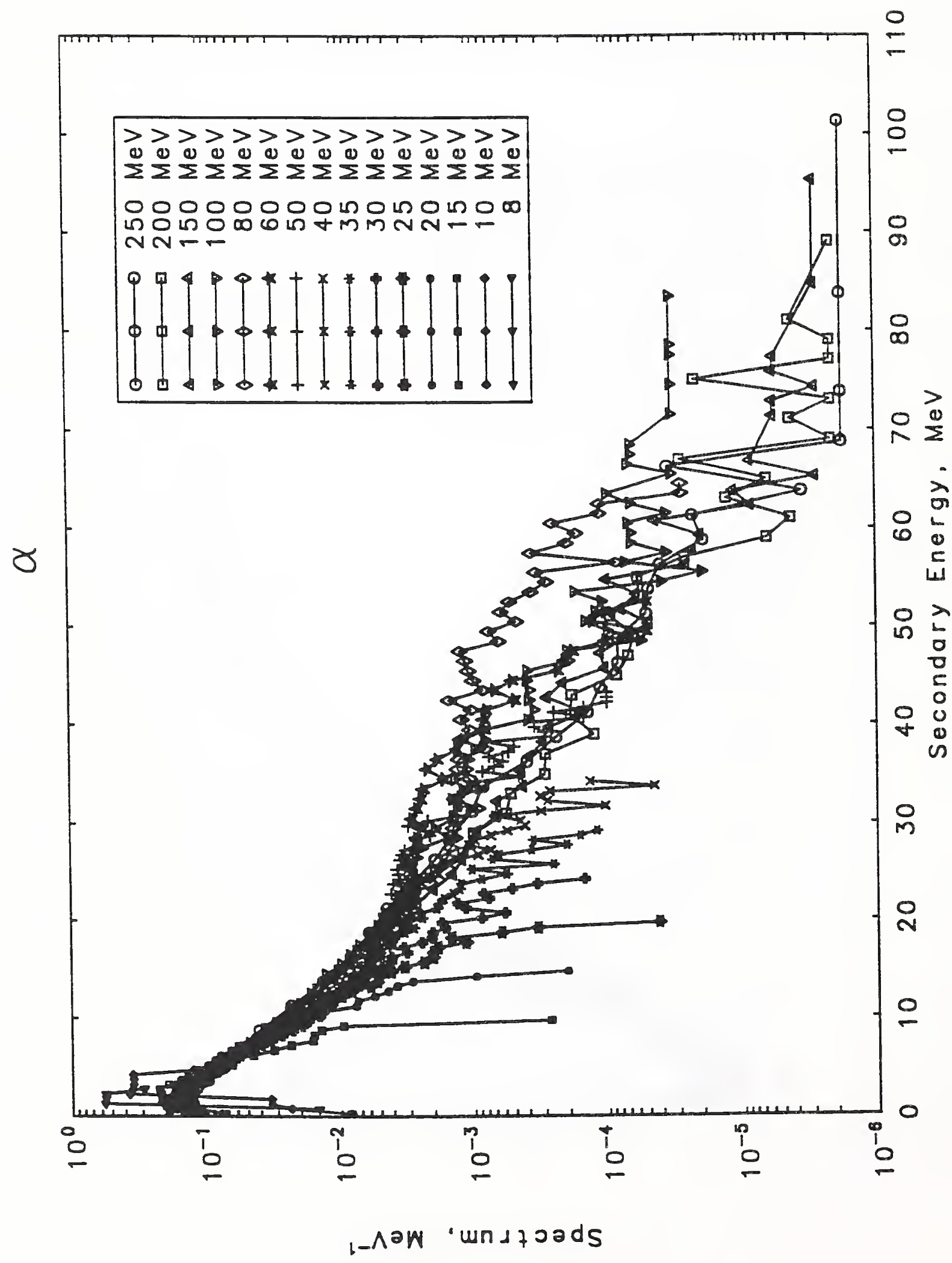

in
$\infty$ 


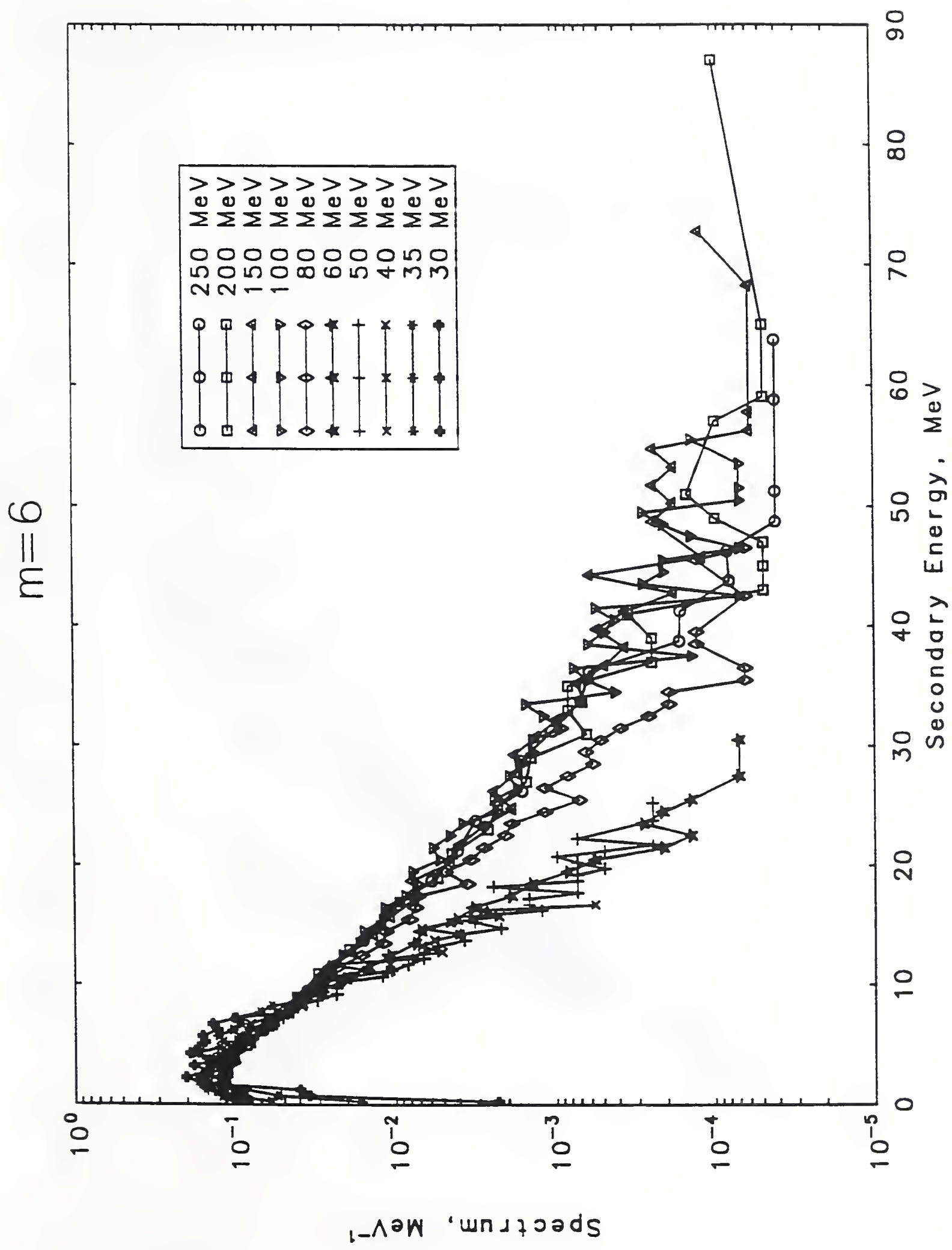

in 


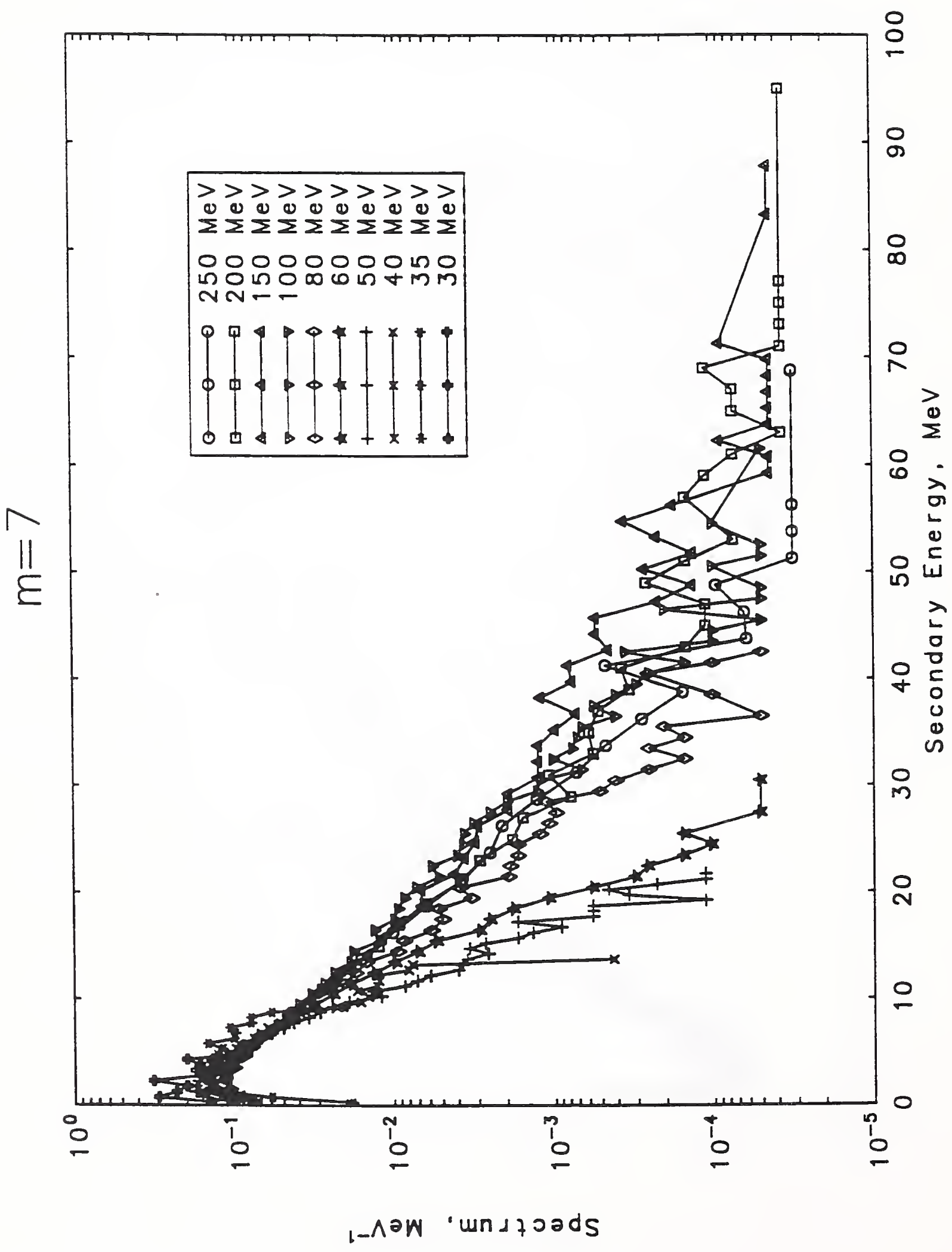

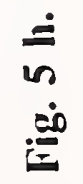




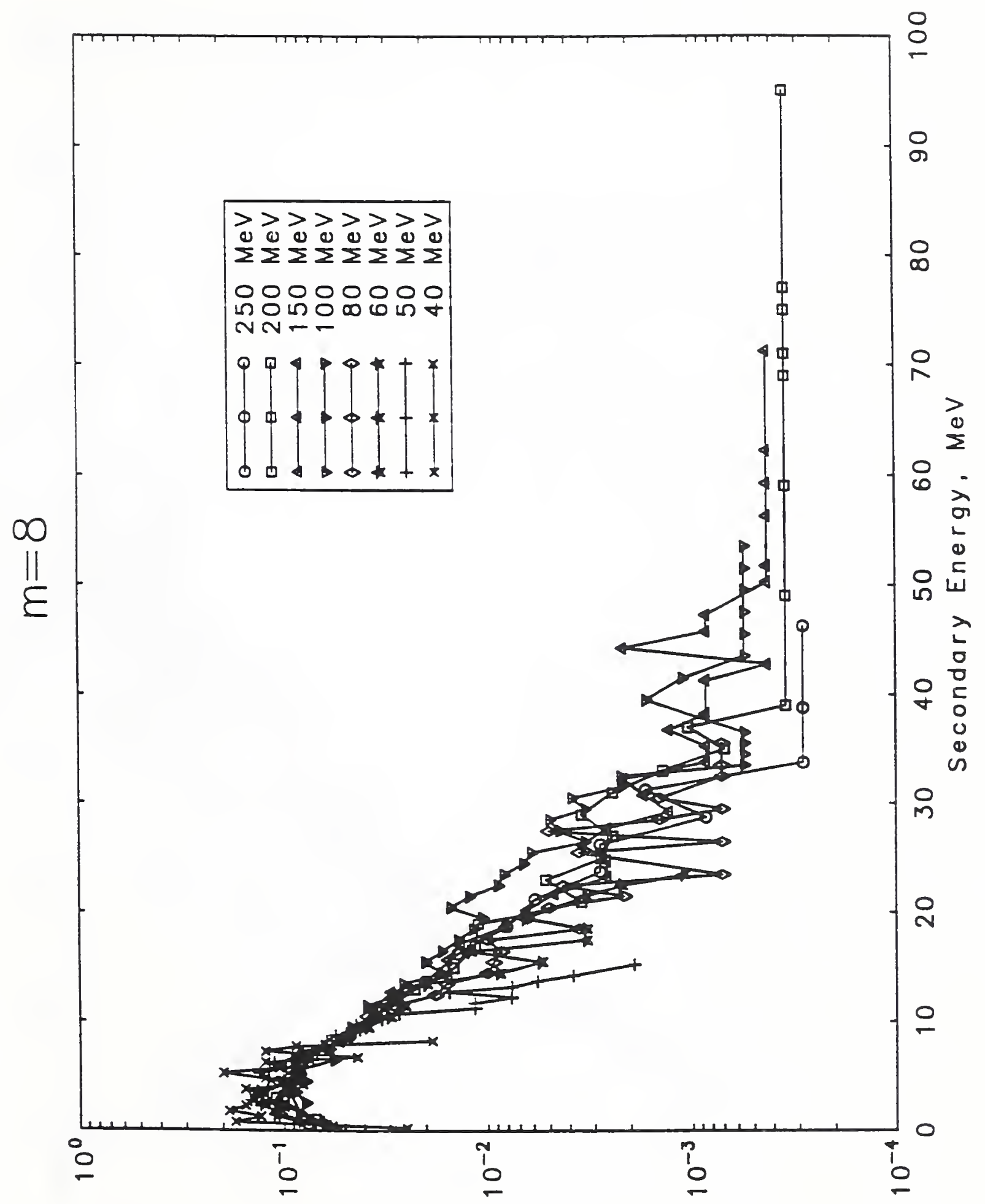

l-^ow 'undzoads 


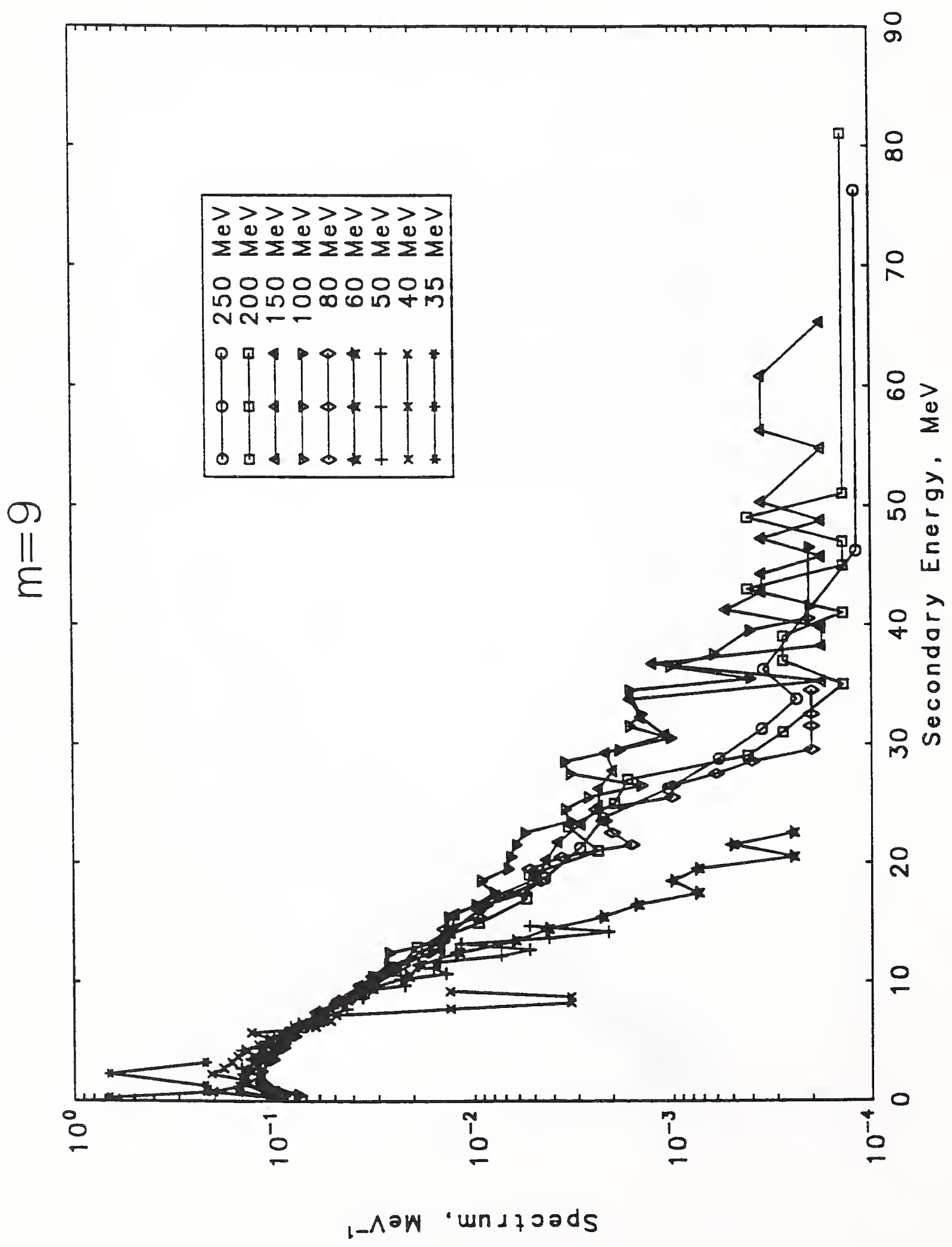

$\dot{m}$ 


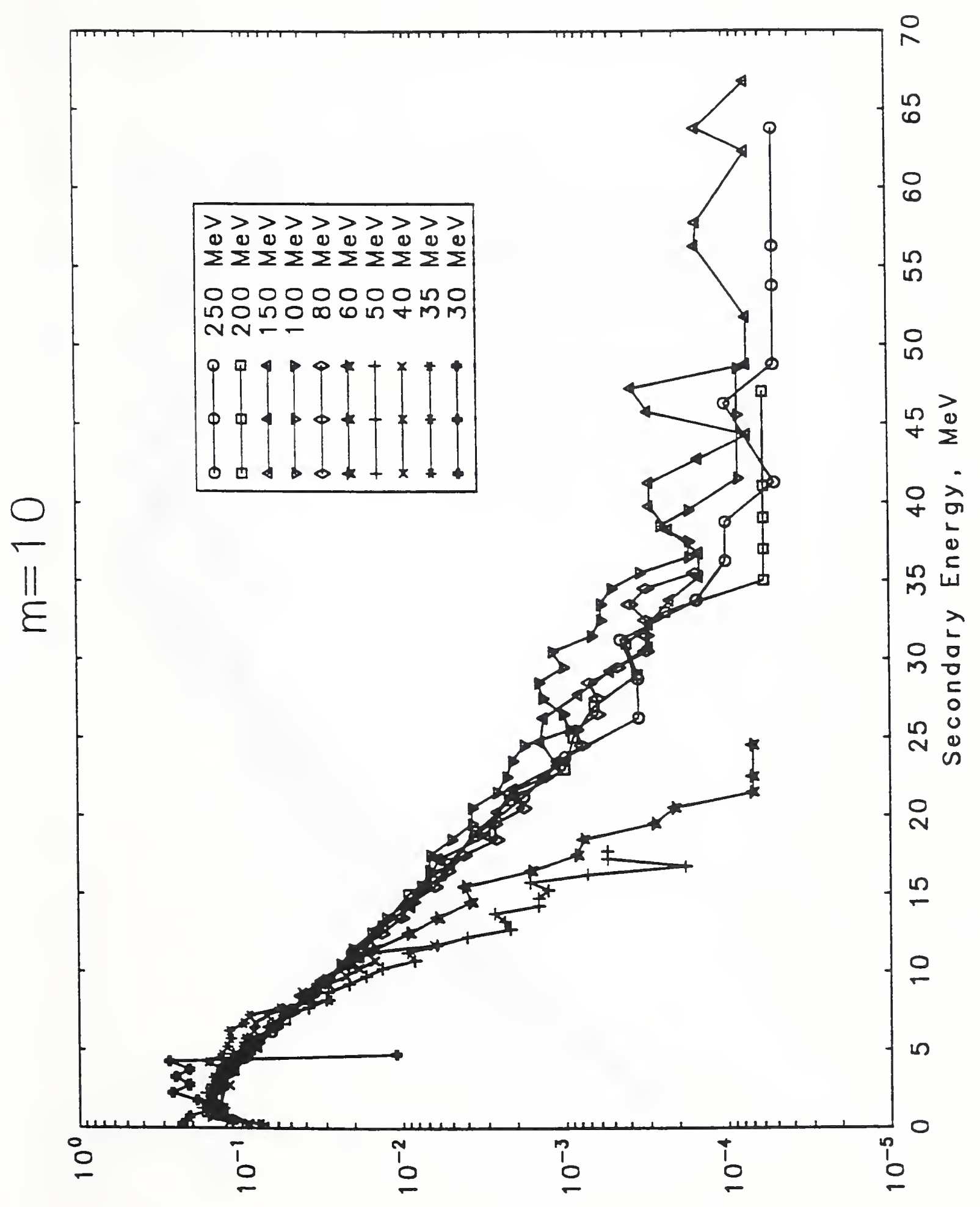

$$
1-\Lambda \partial W \cdot u n d z o a d s
$$




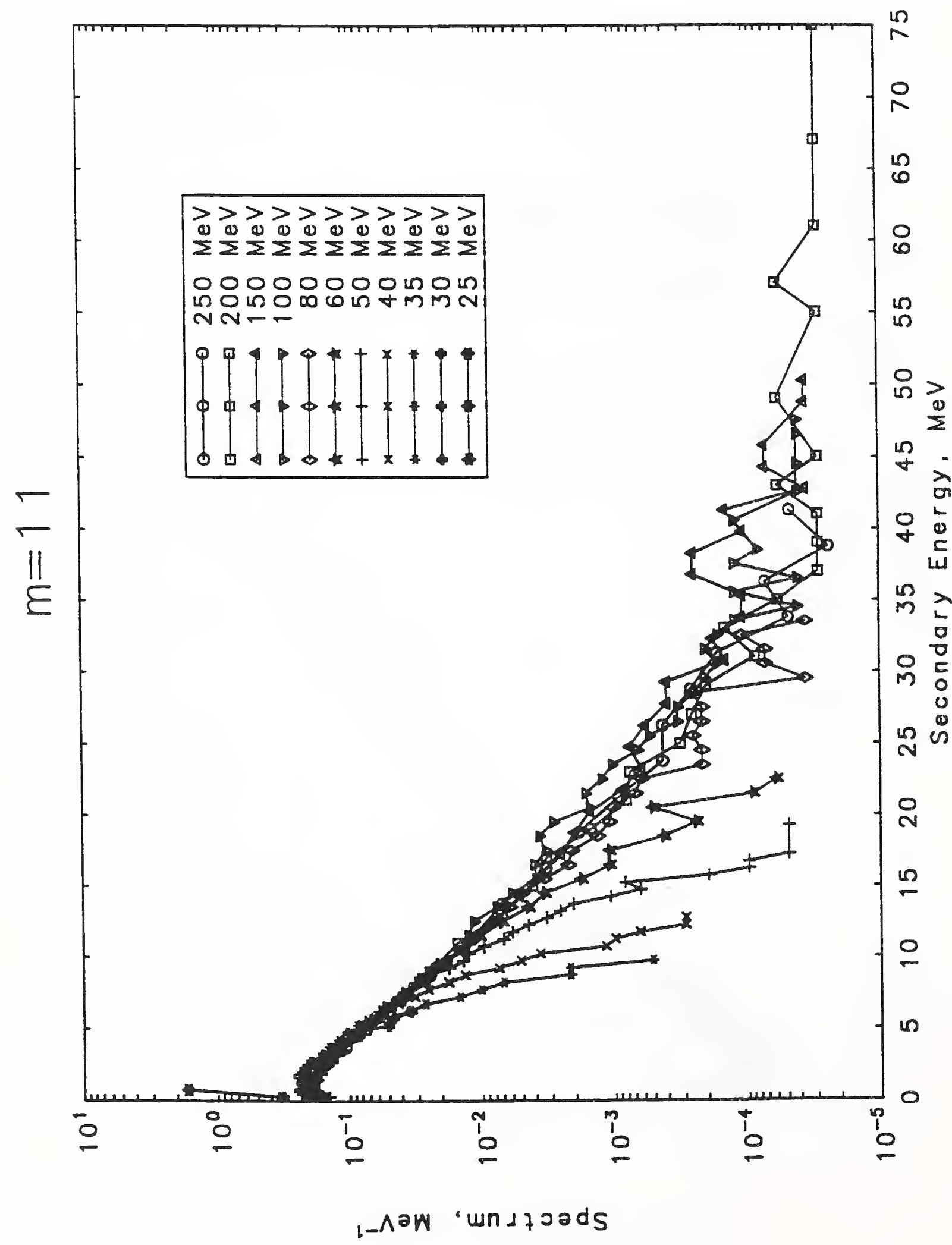

$\frac{10}{i 0}$ 


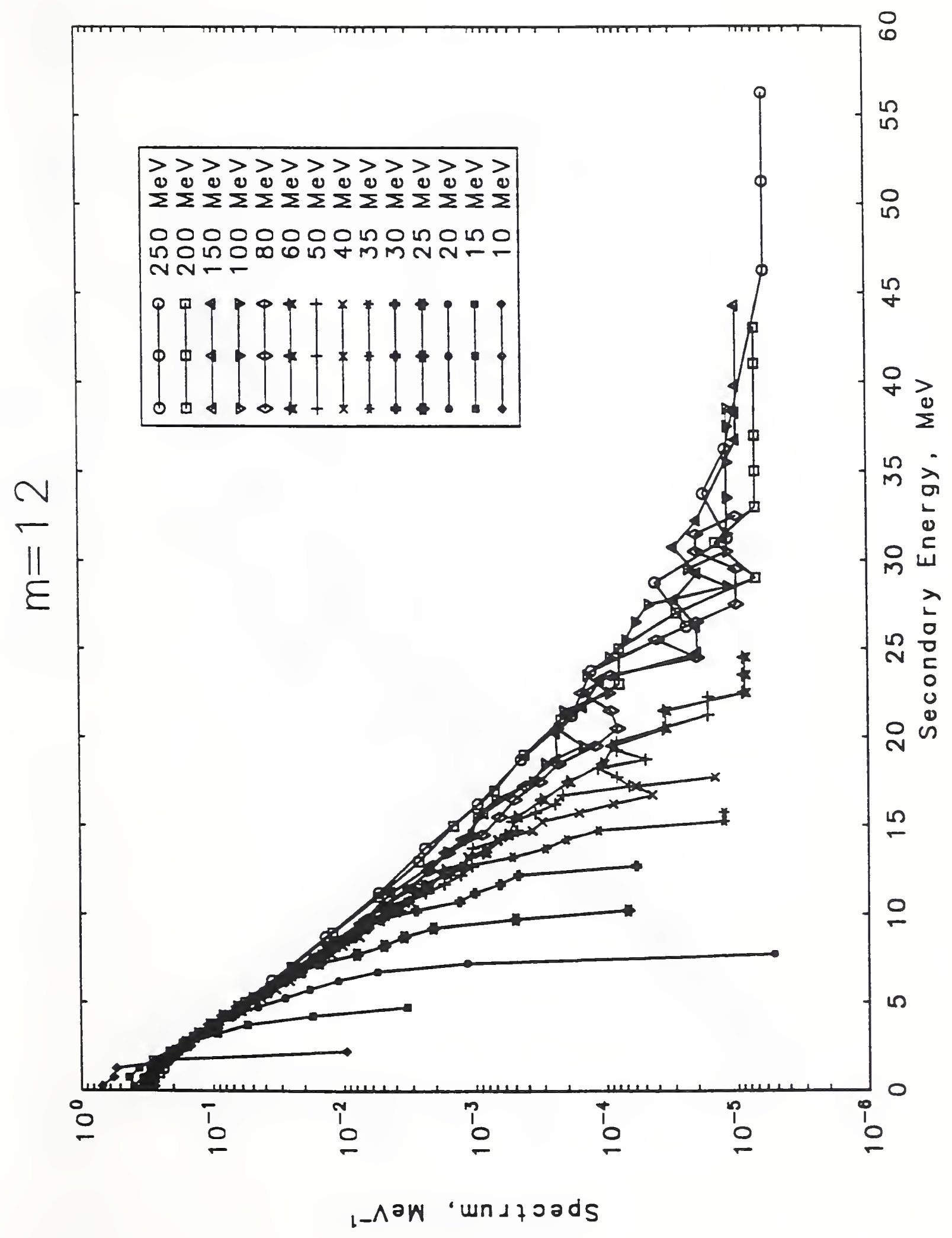

$\dot{0}$
$\dot{0}$ 


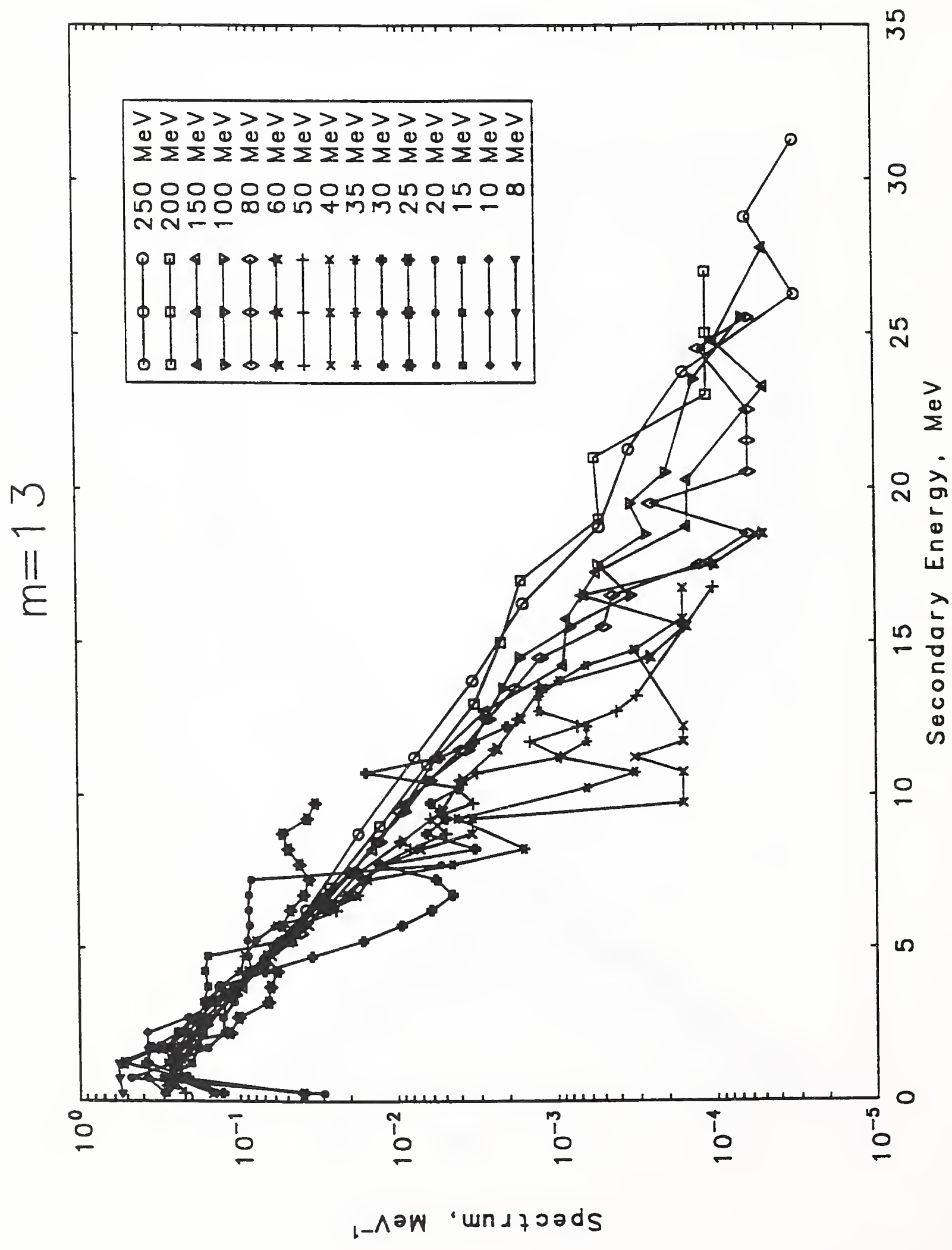

$\vdots$
in
$\vdots 0$ 


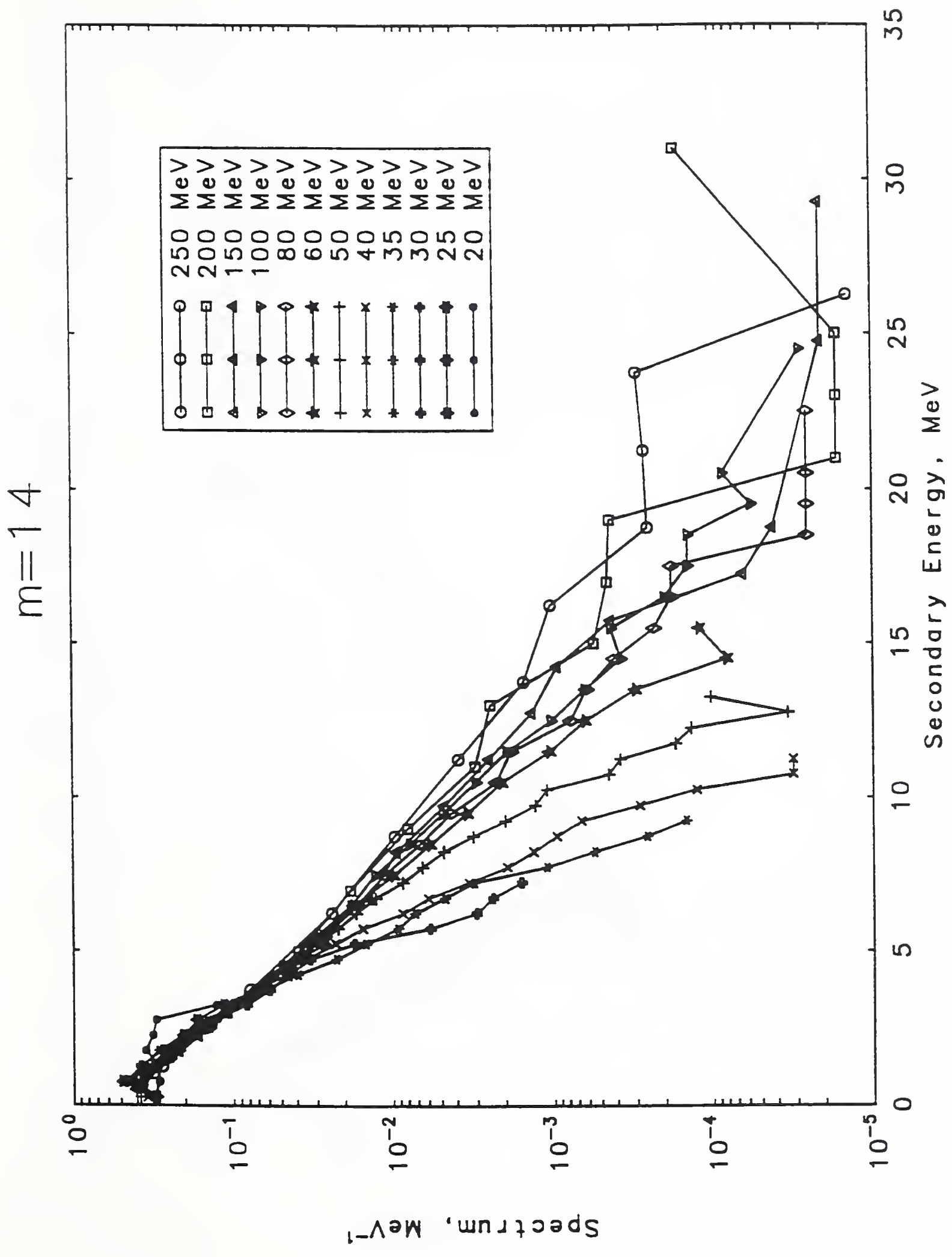

0
in
0 


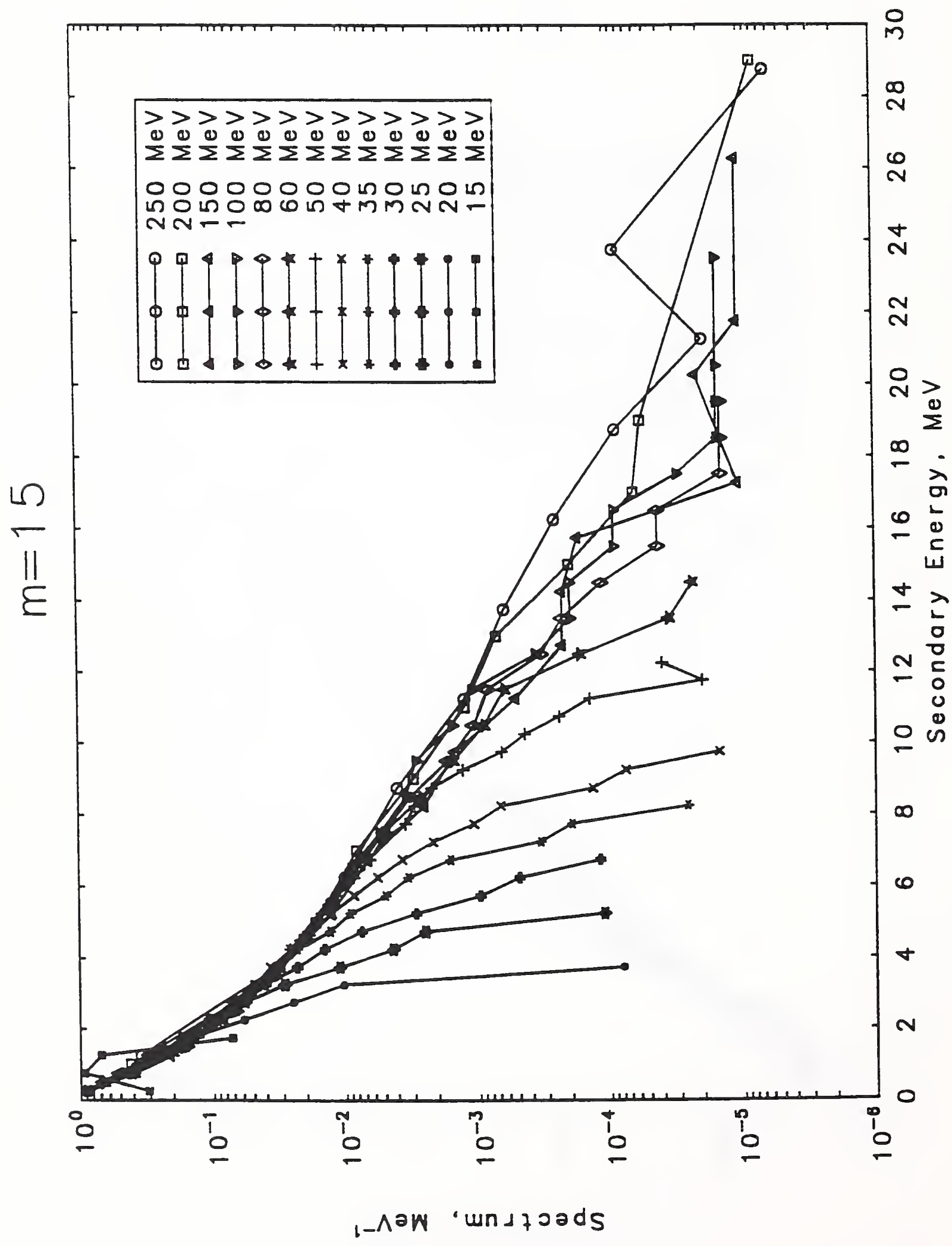

$\dot{5}$
in
$\dot{0}$ 


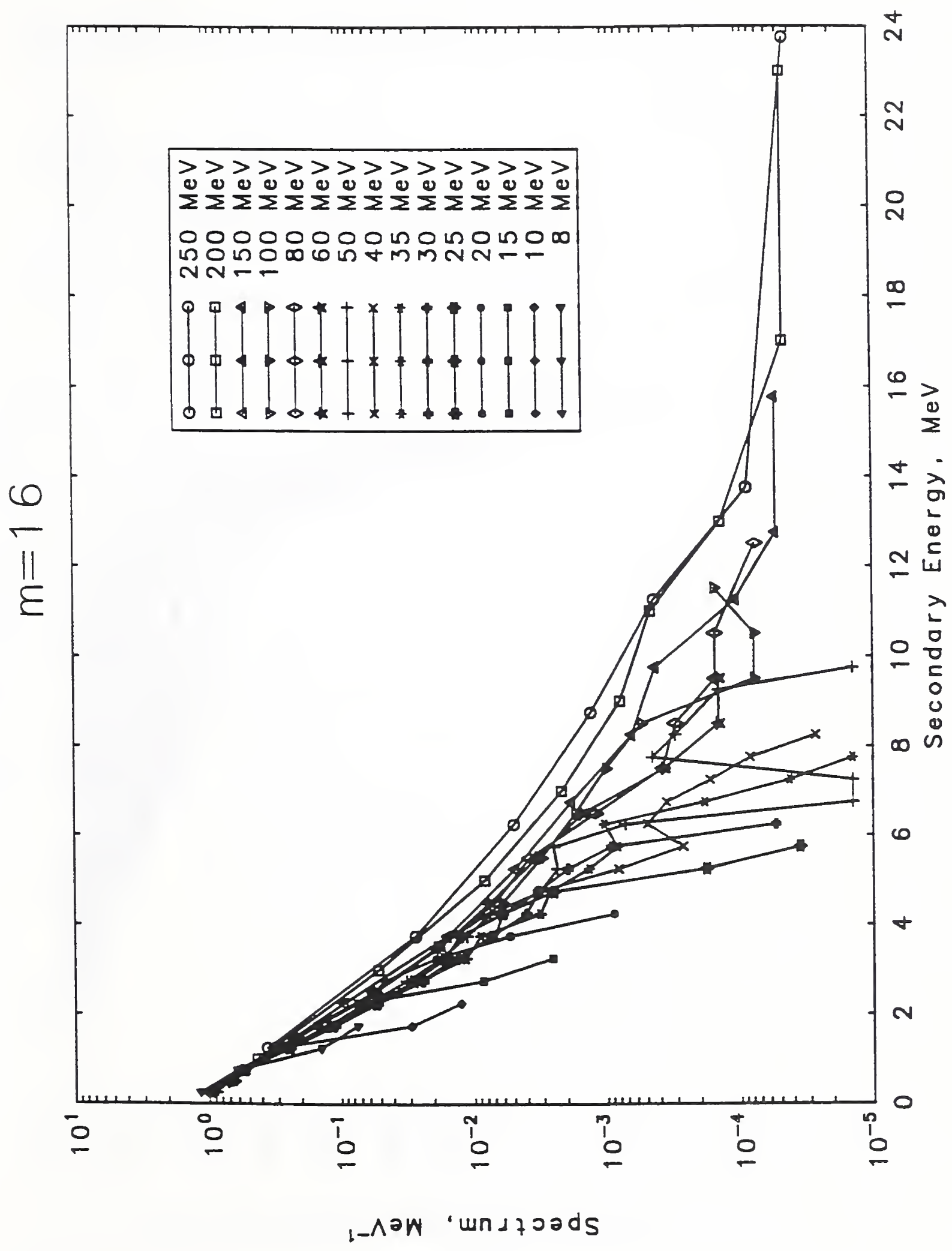




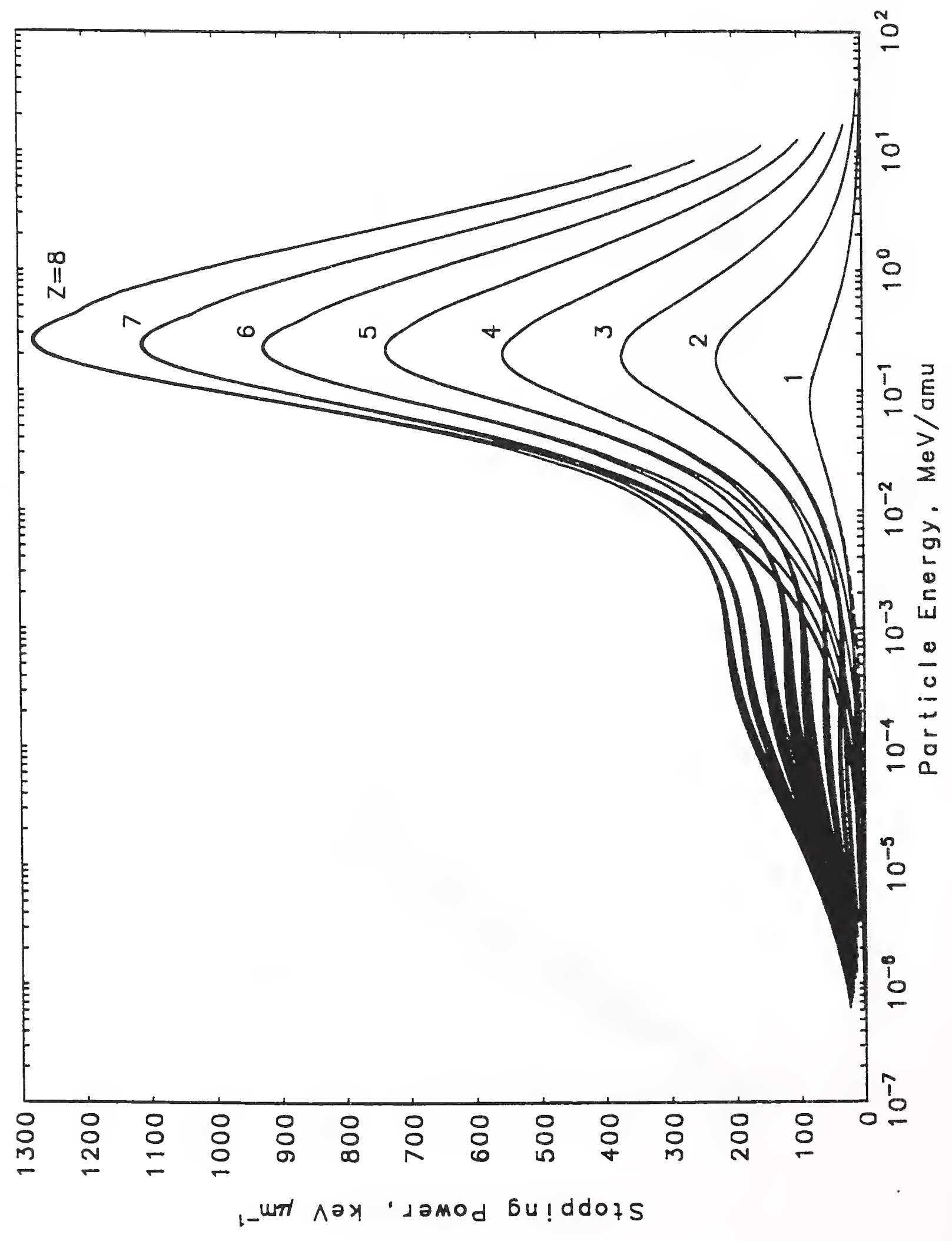

$\infty$ 


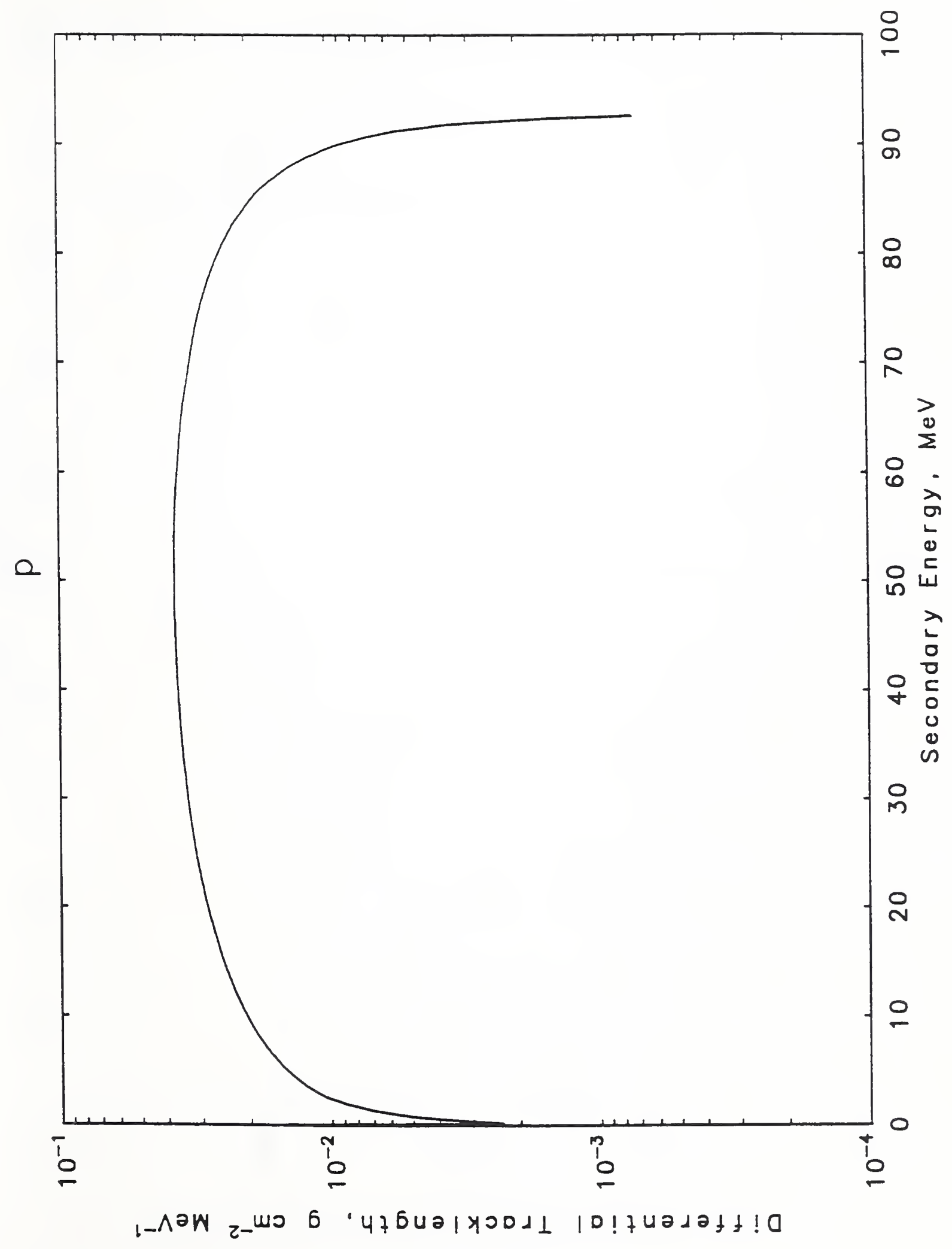




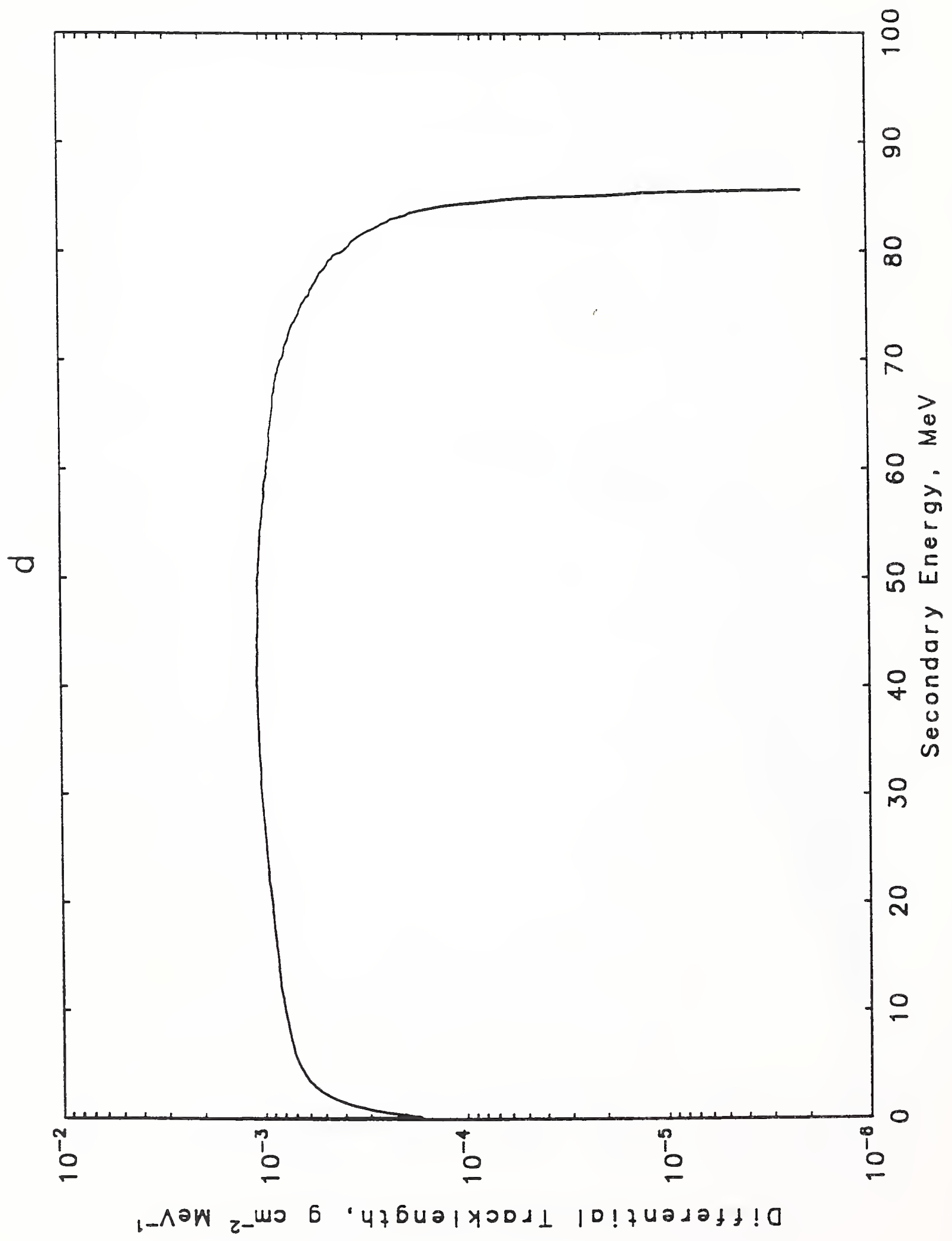

$\stackrel{\dot{0}}{0}$ 


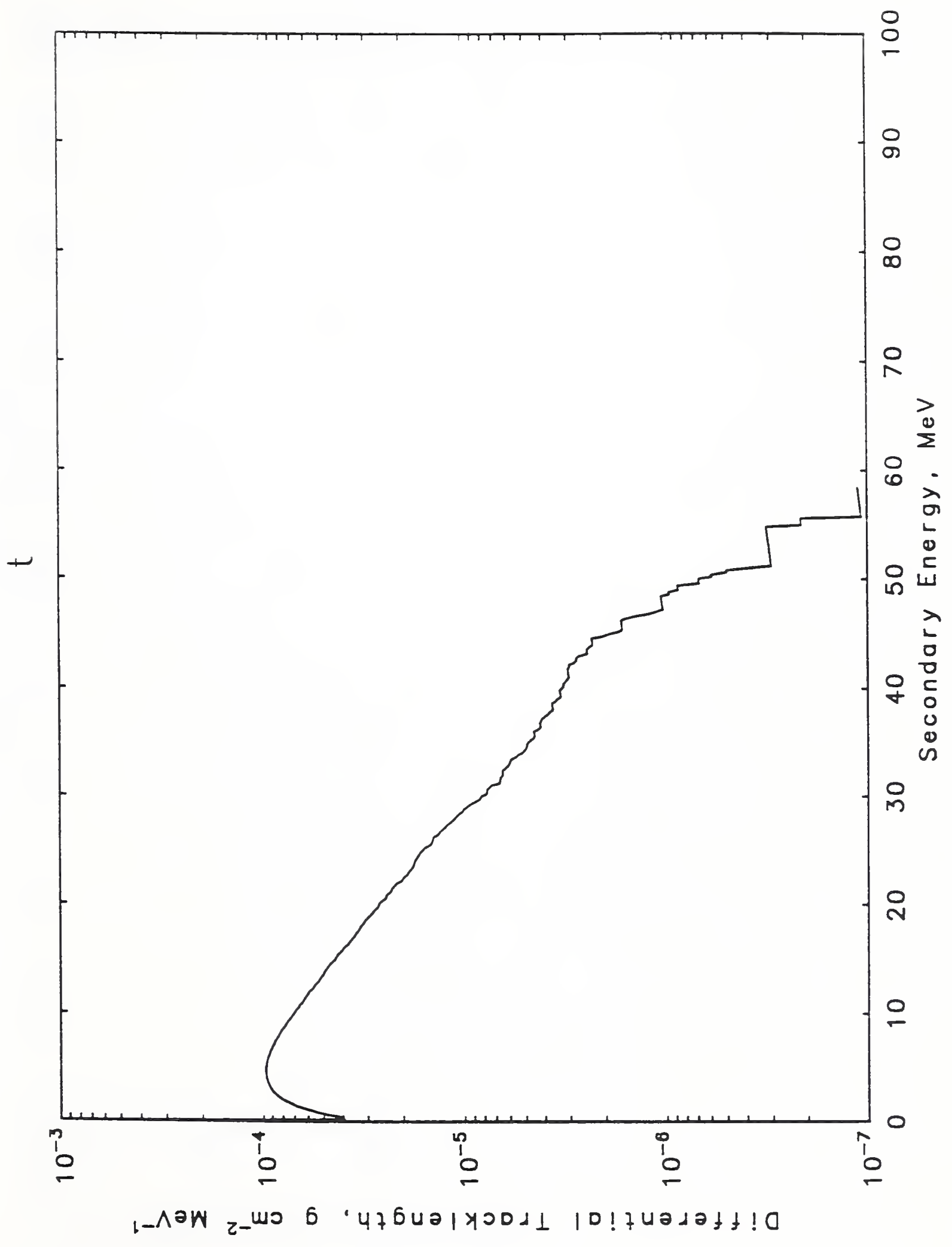




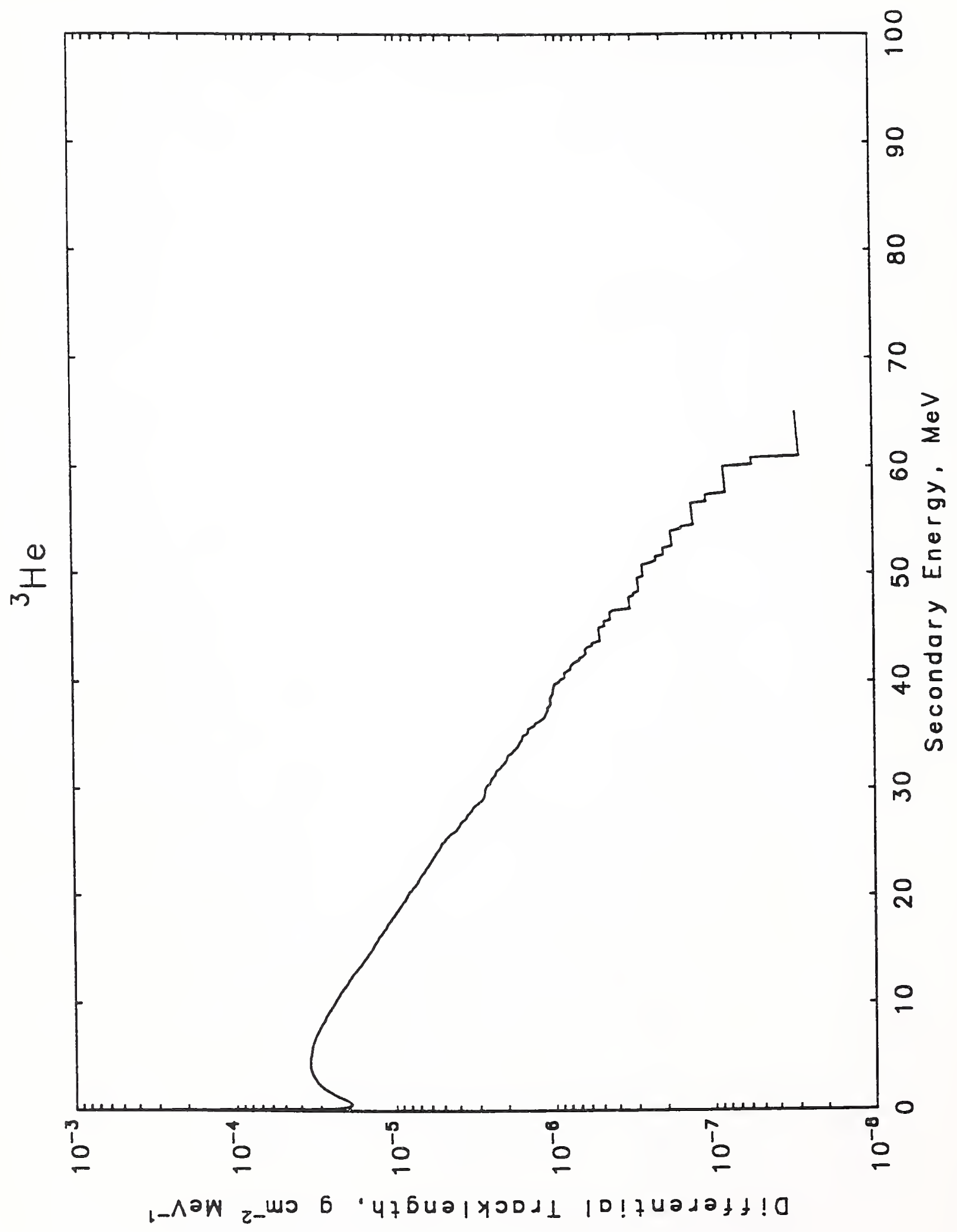

递 


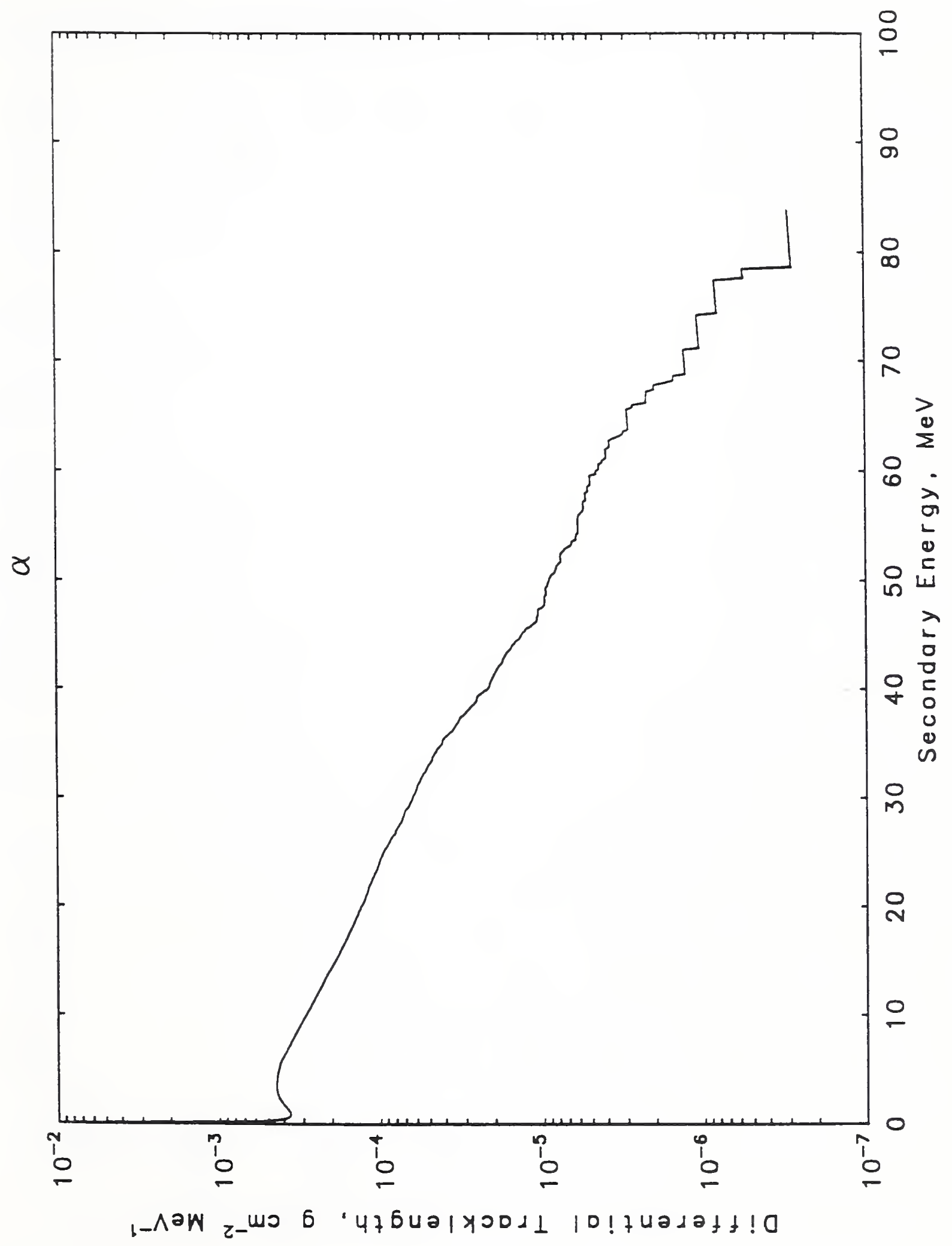

i 


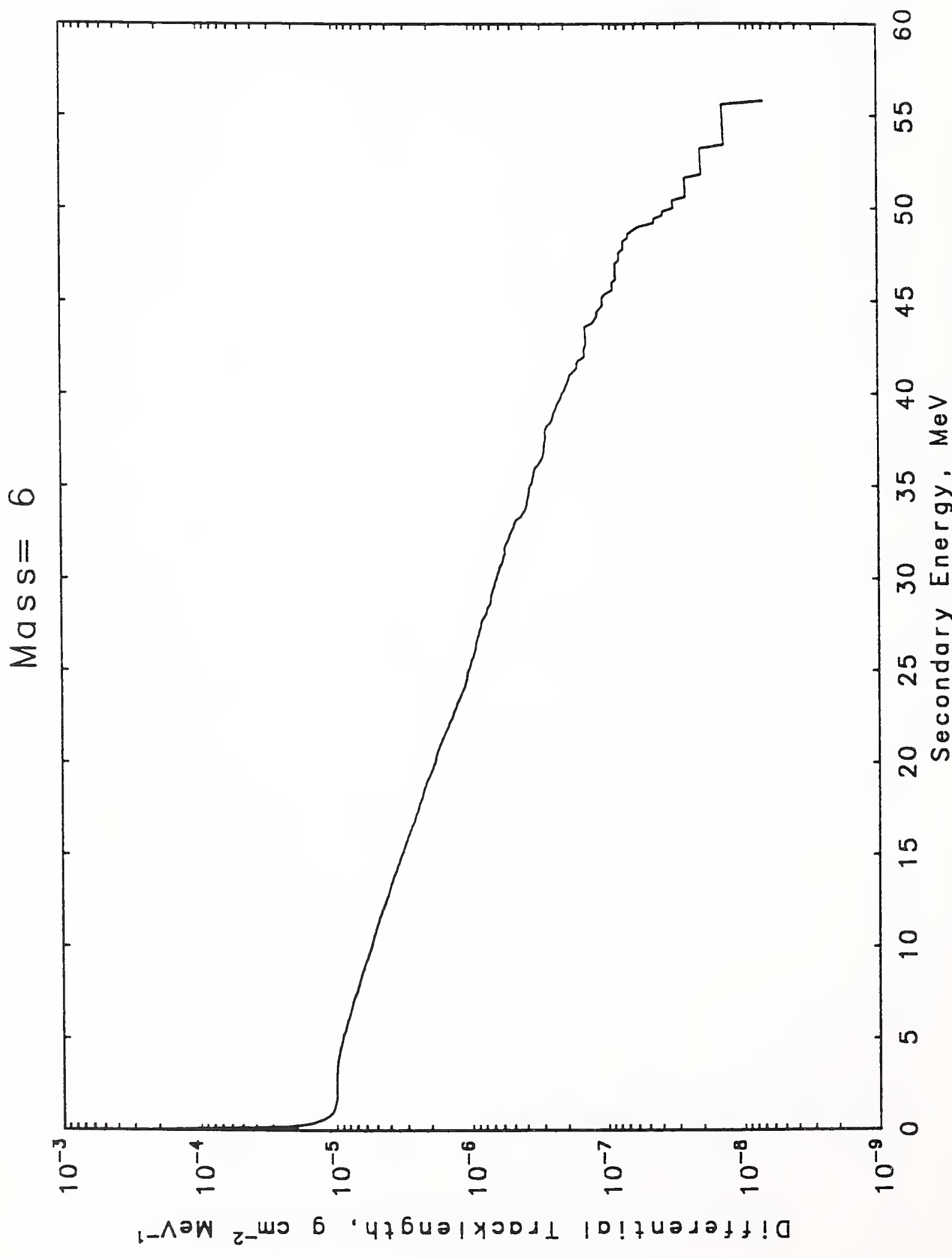

$\stackrel{2}{2}$

60 


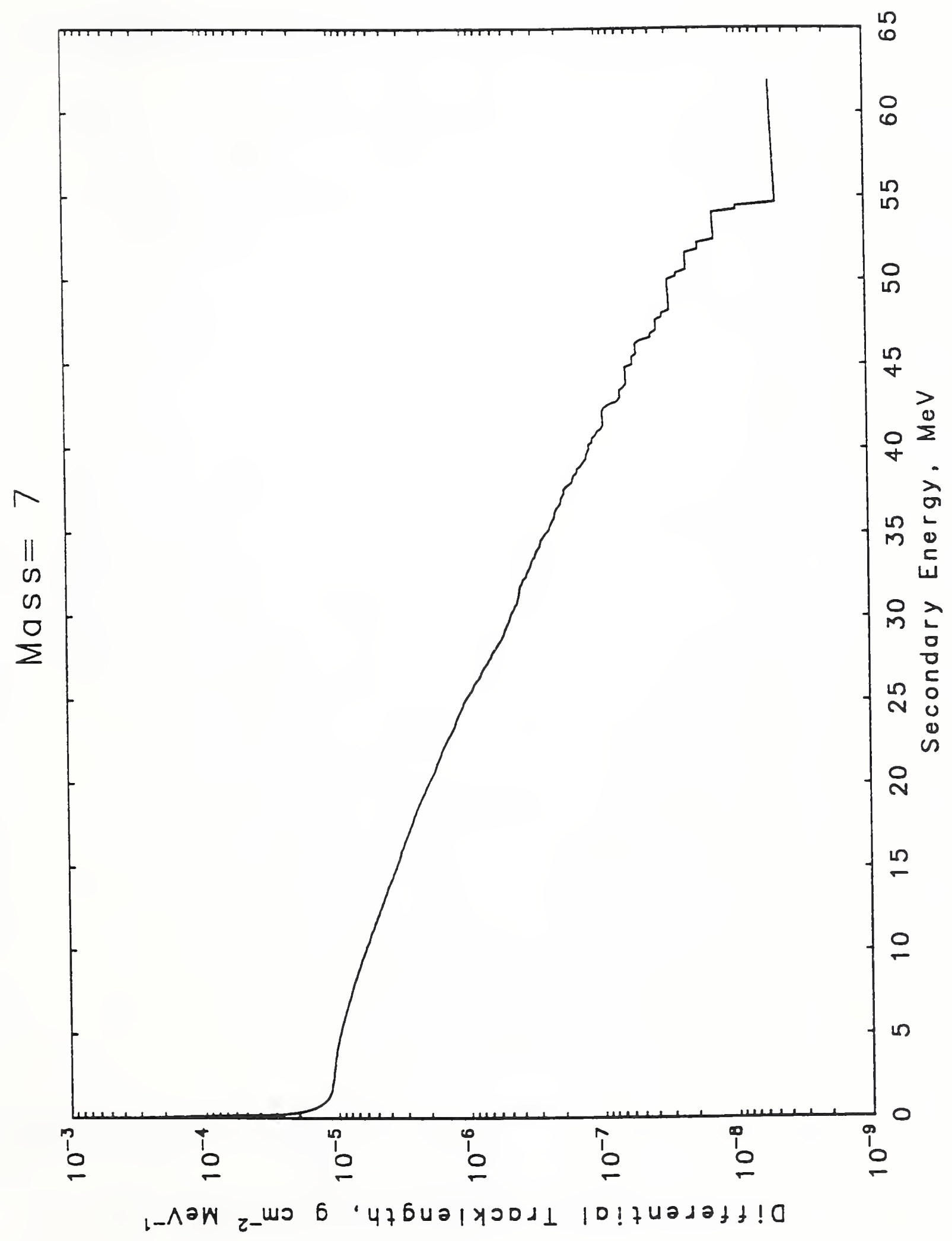

io 


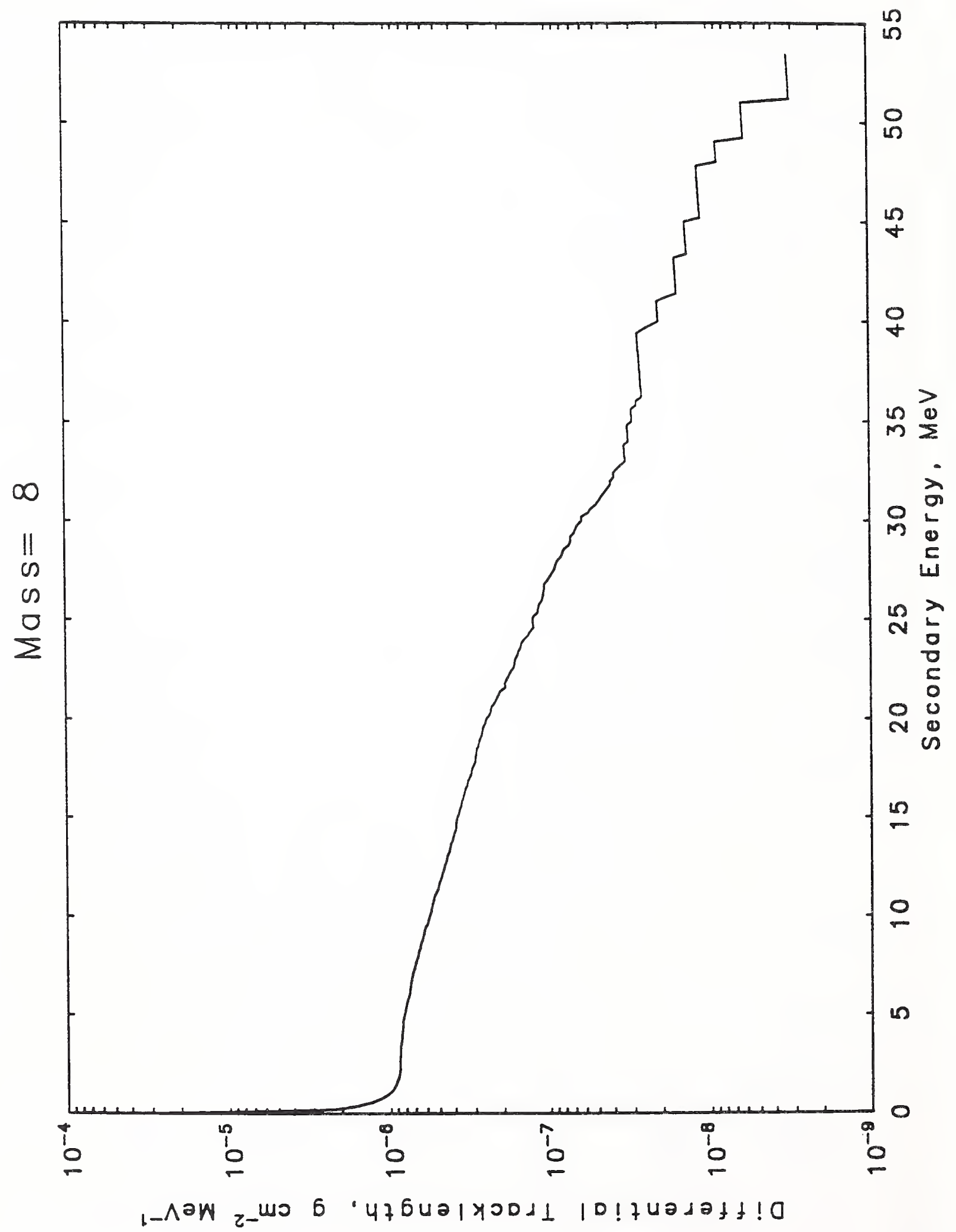

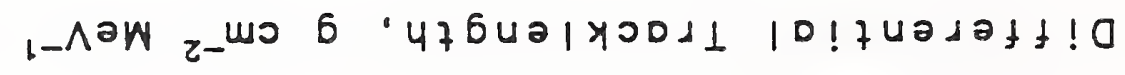




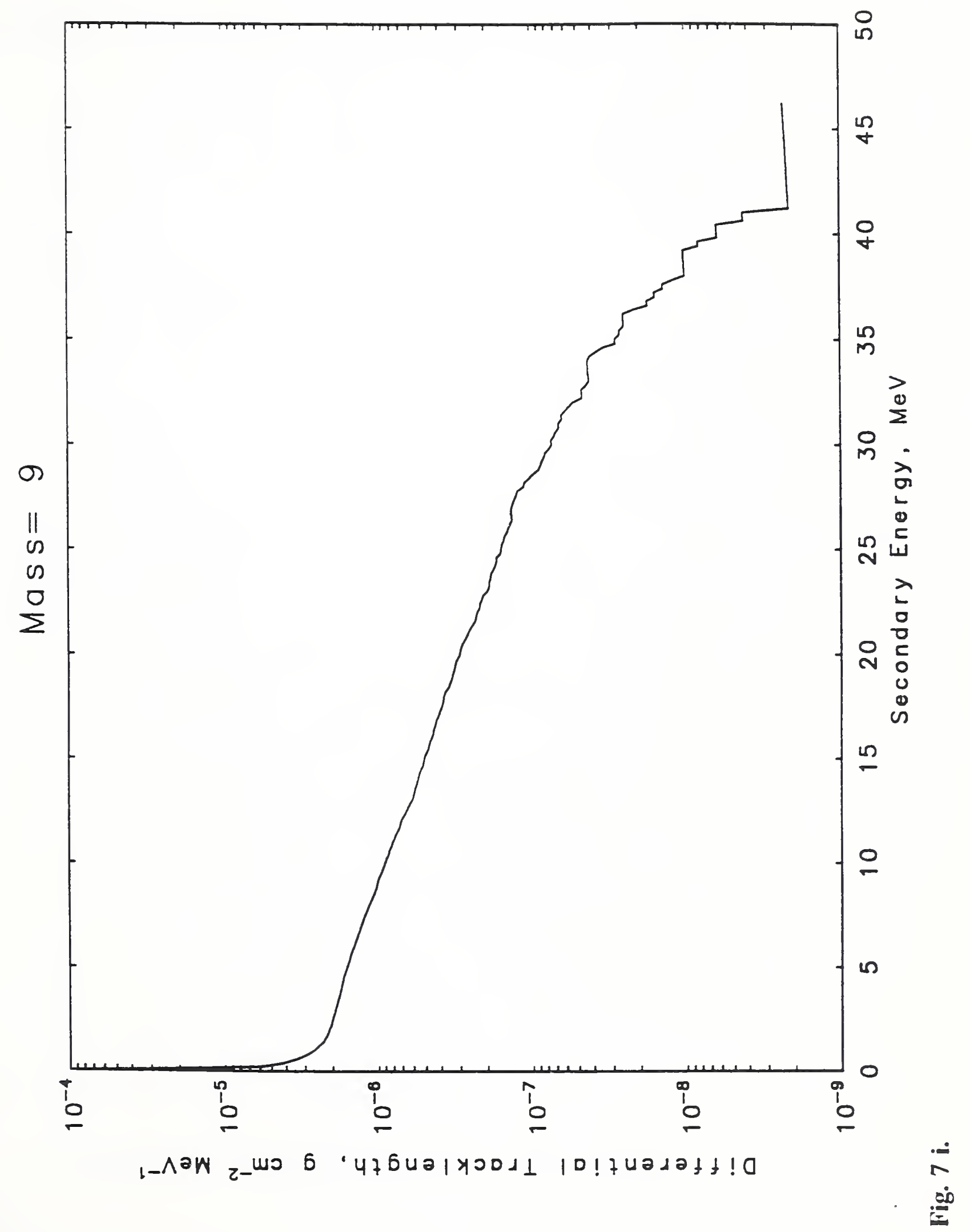

63 


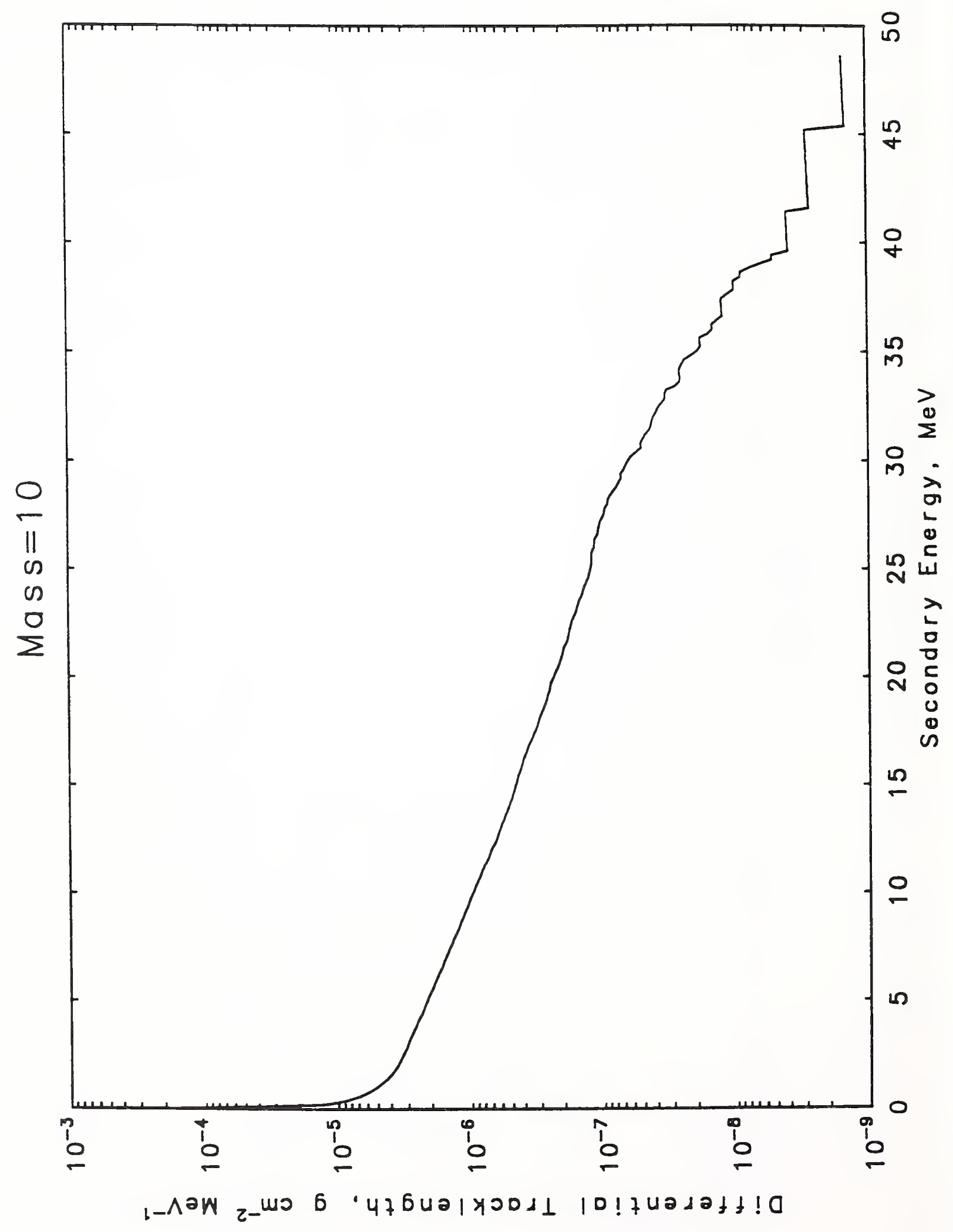




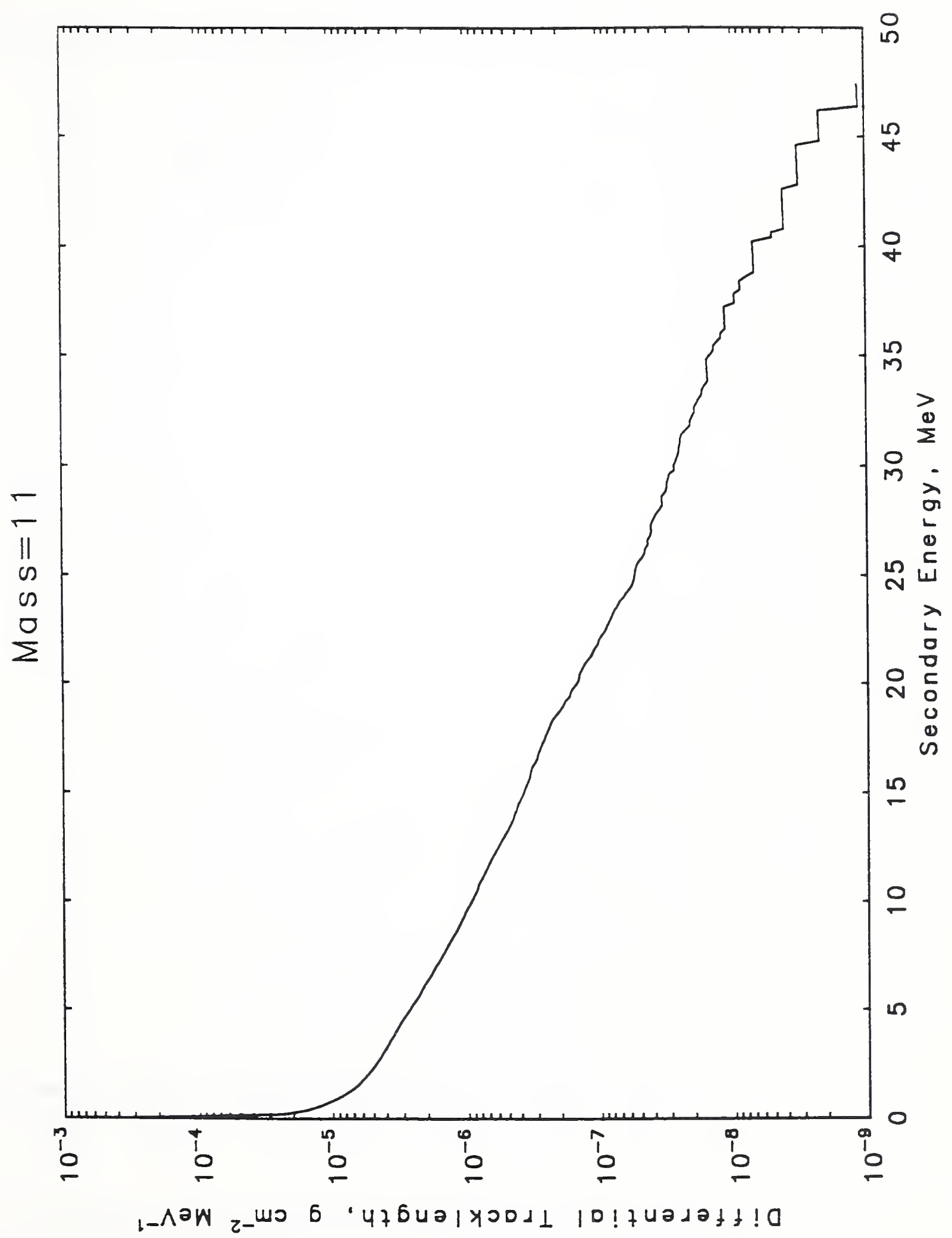




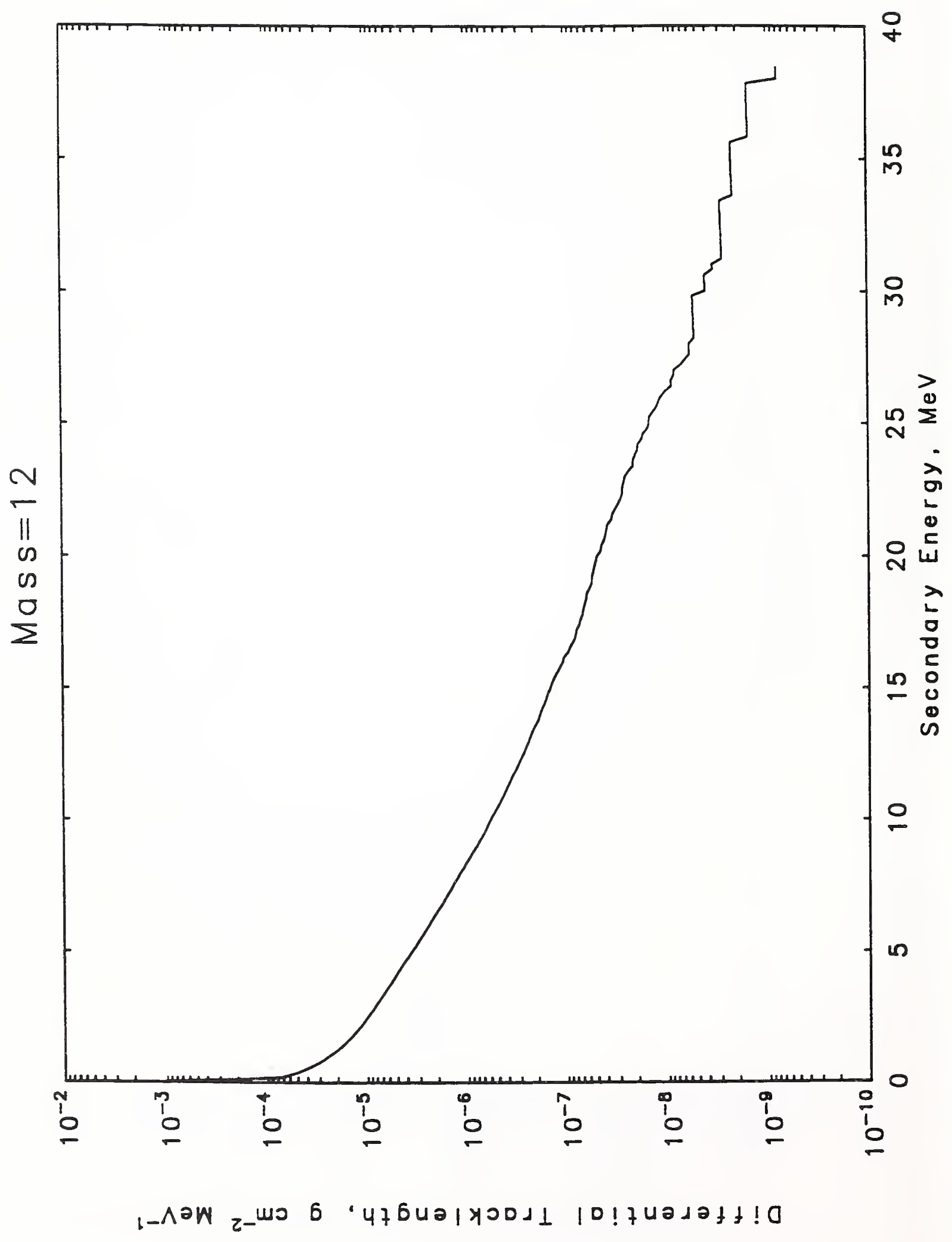

$\ddot{n}$ 


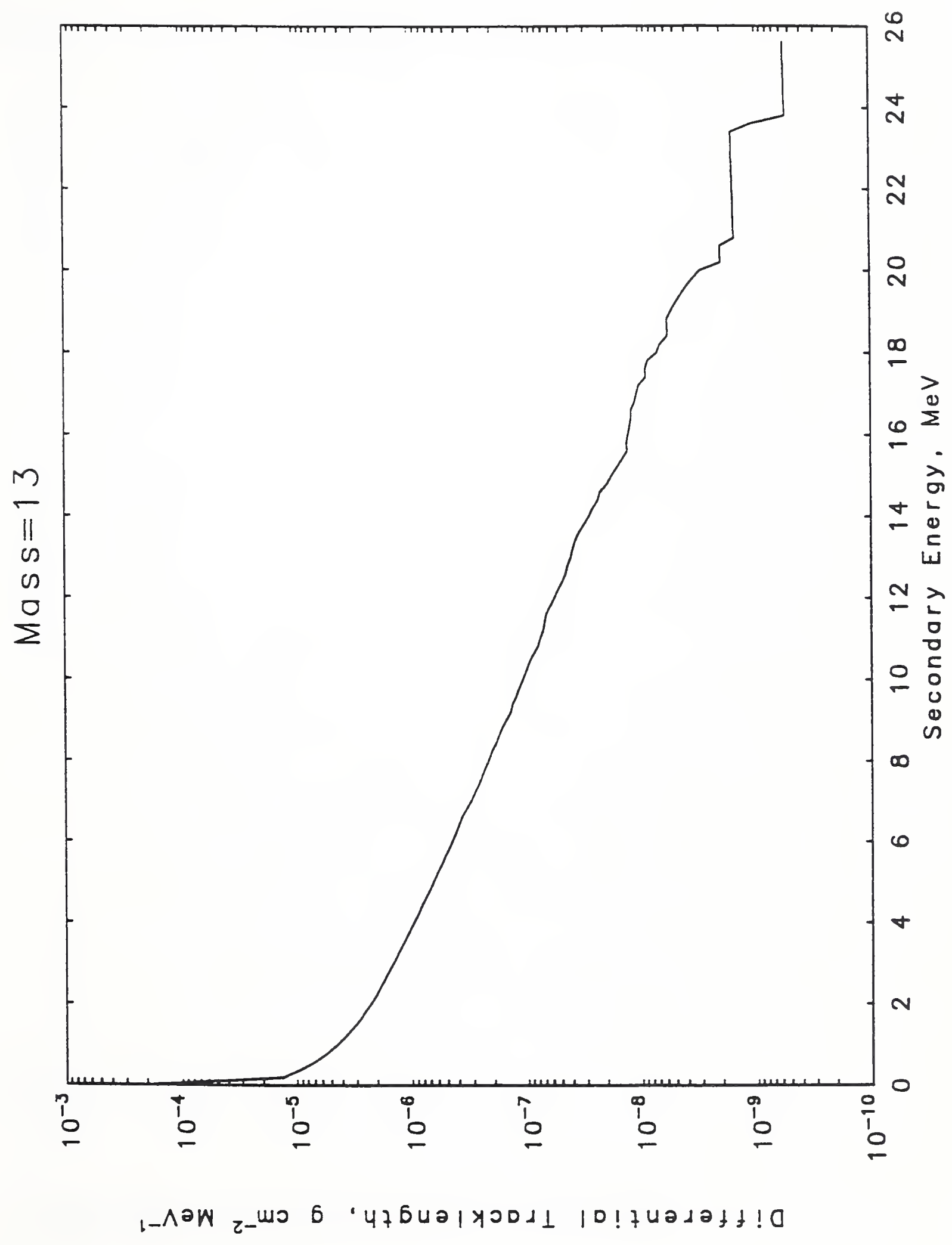

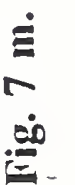




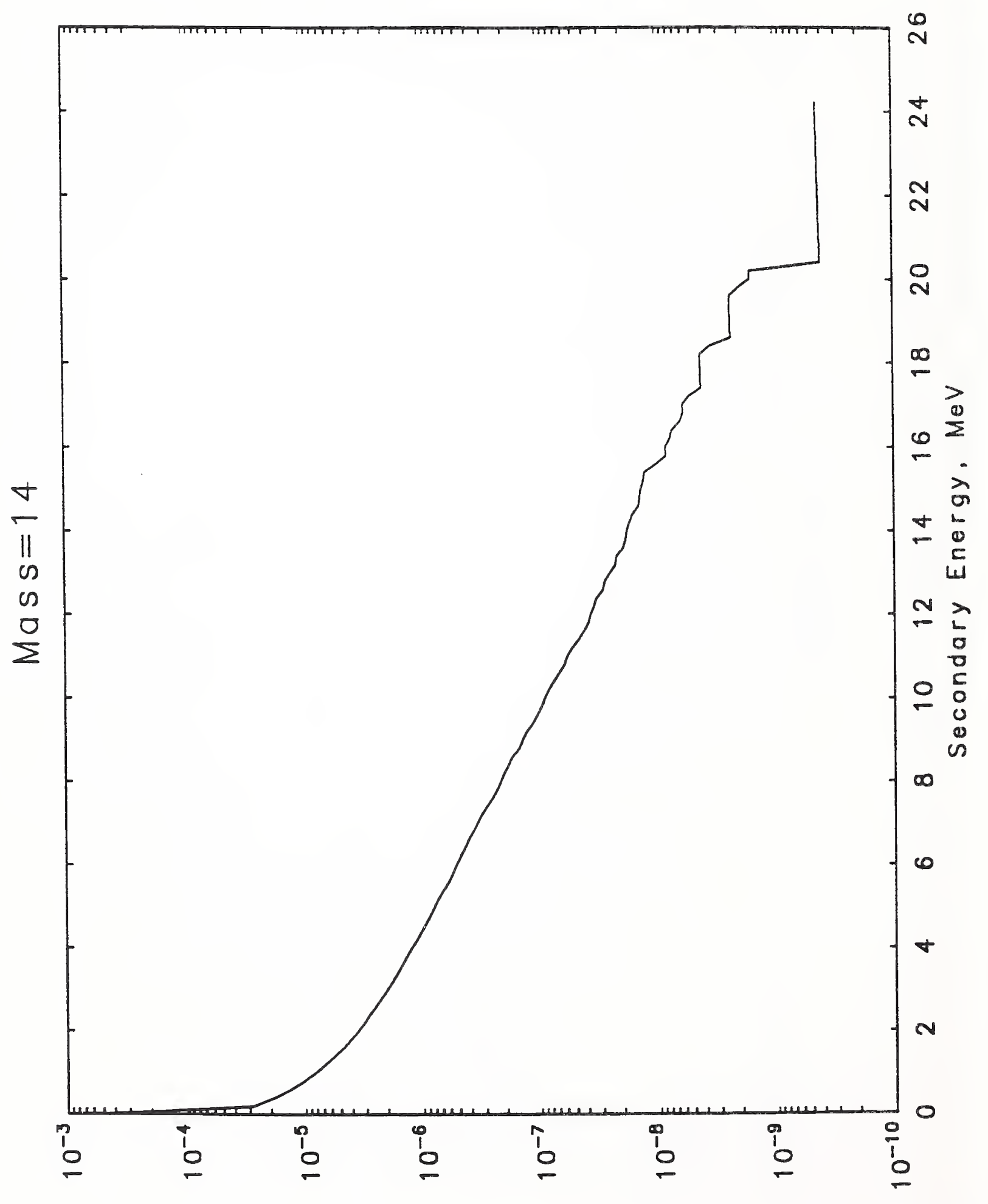

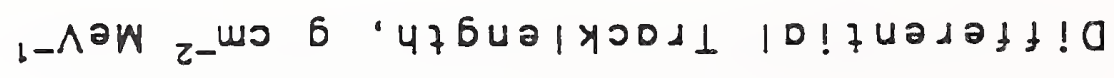




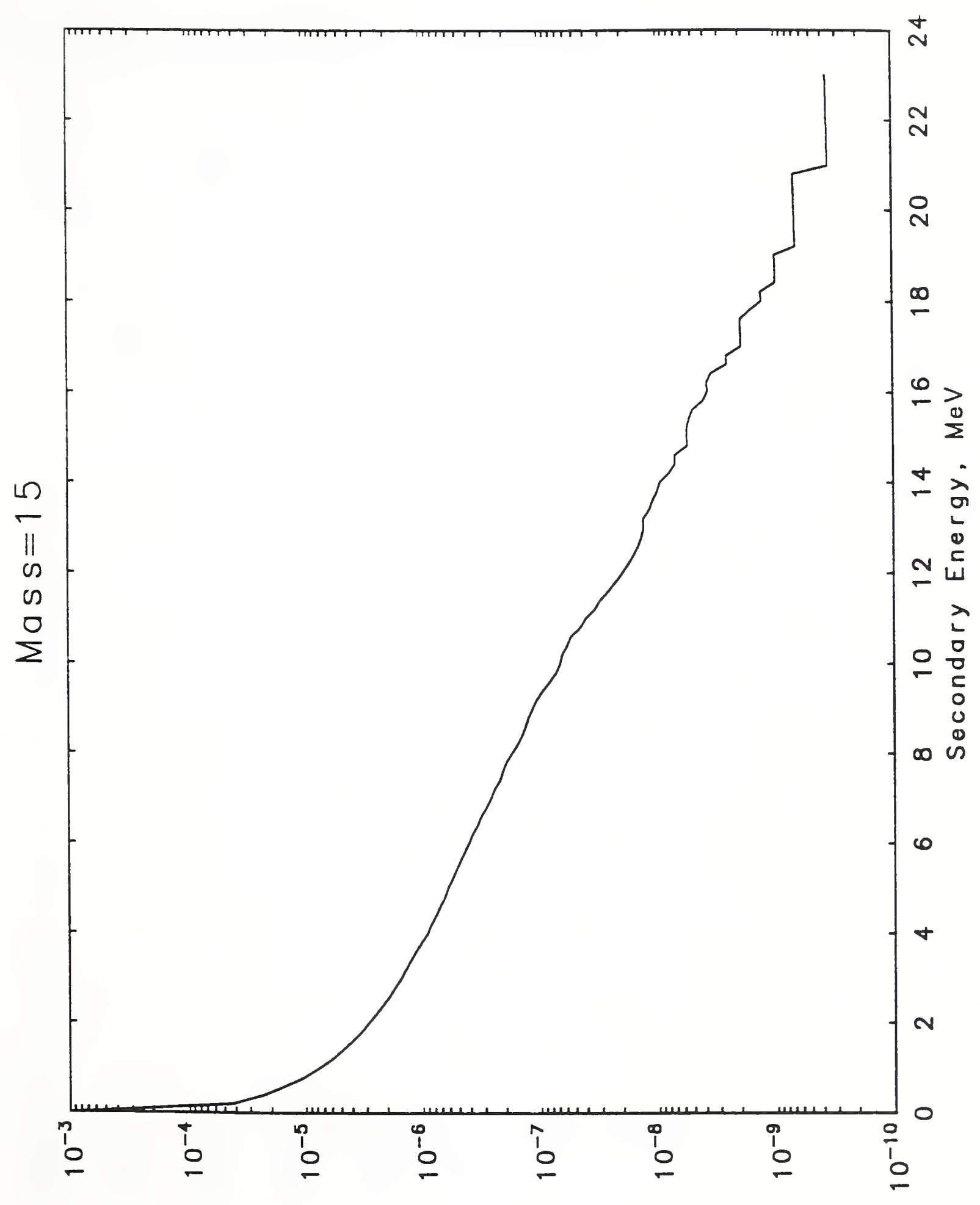

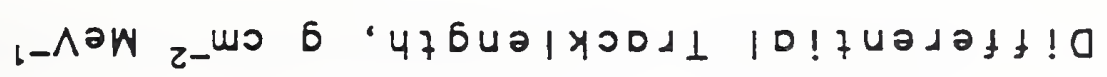




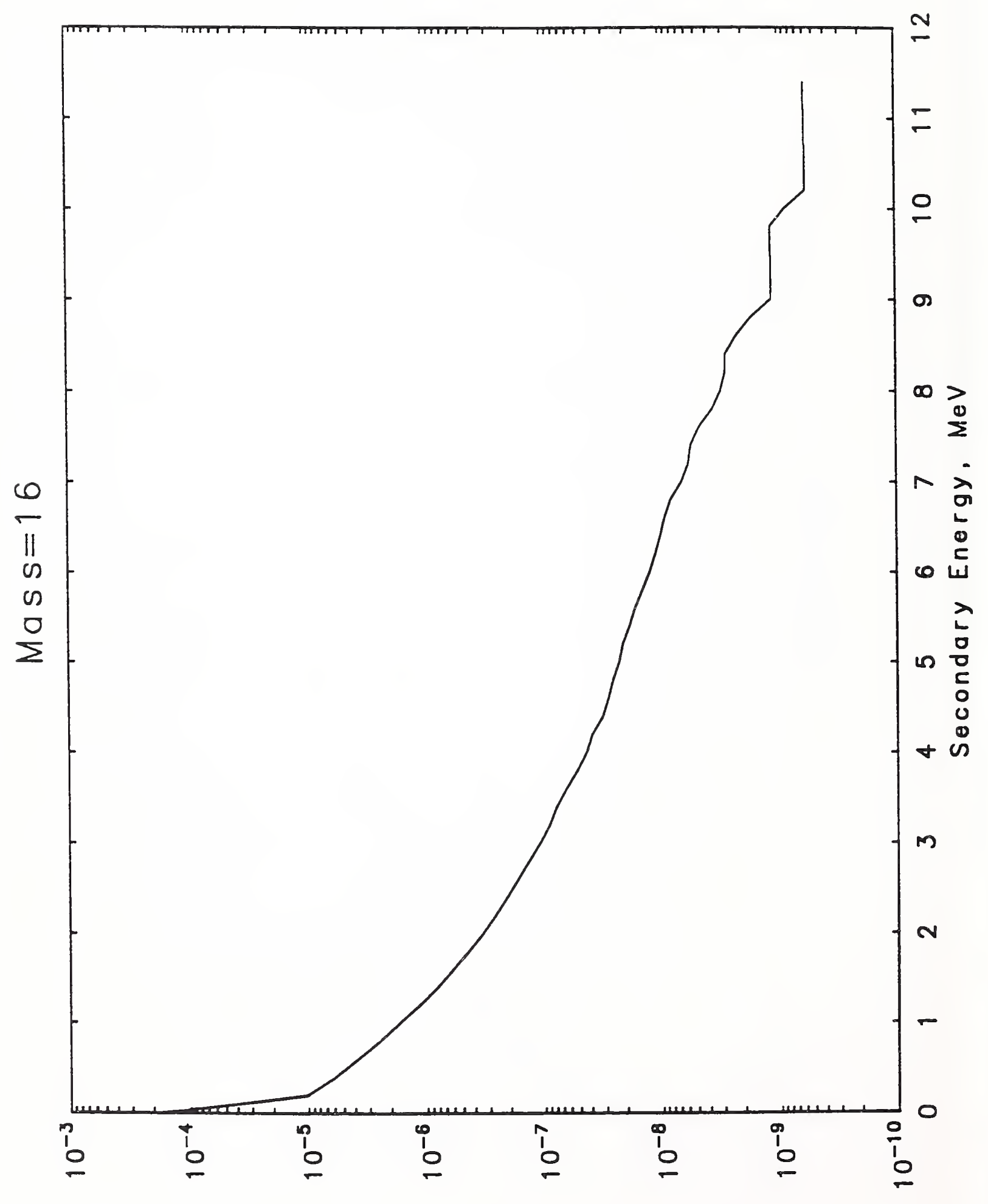

เภลN z-ய0 6 ' 


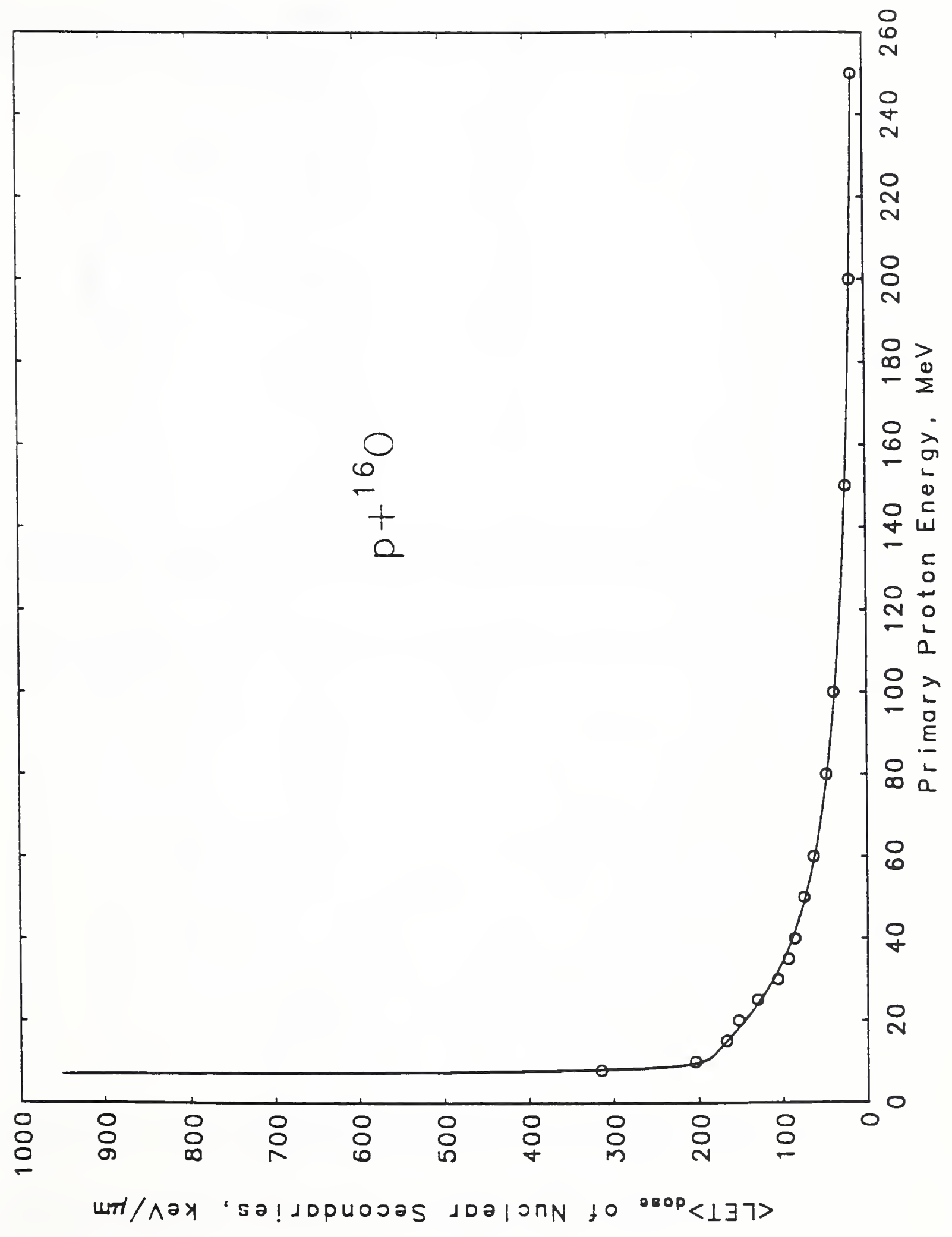



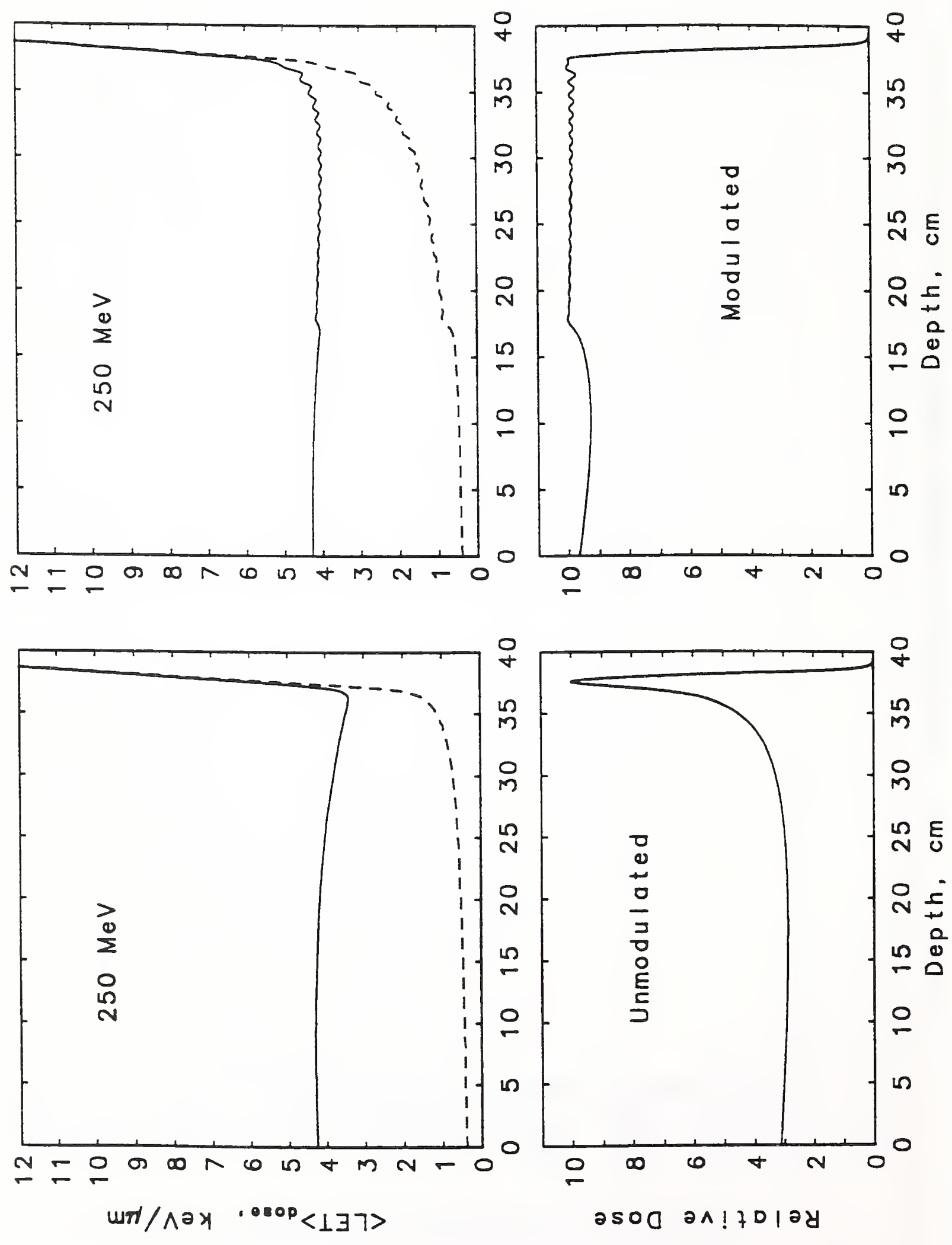

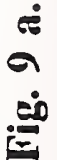



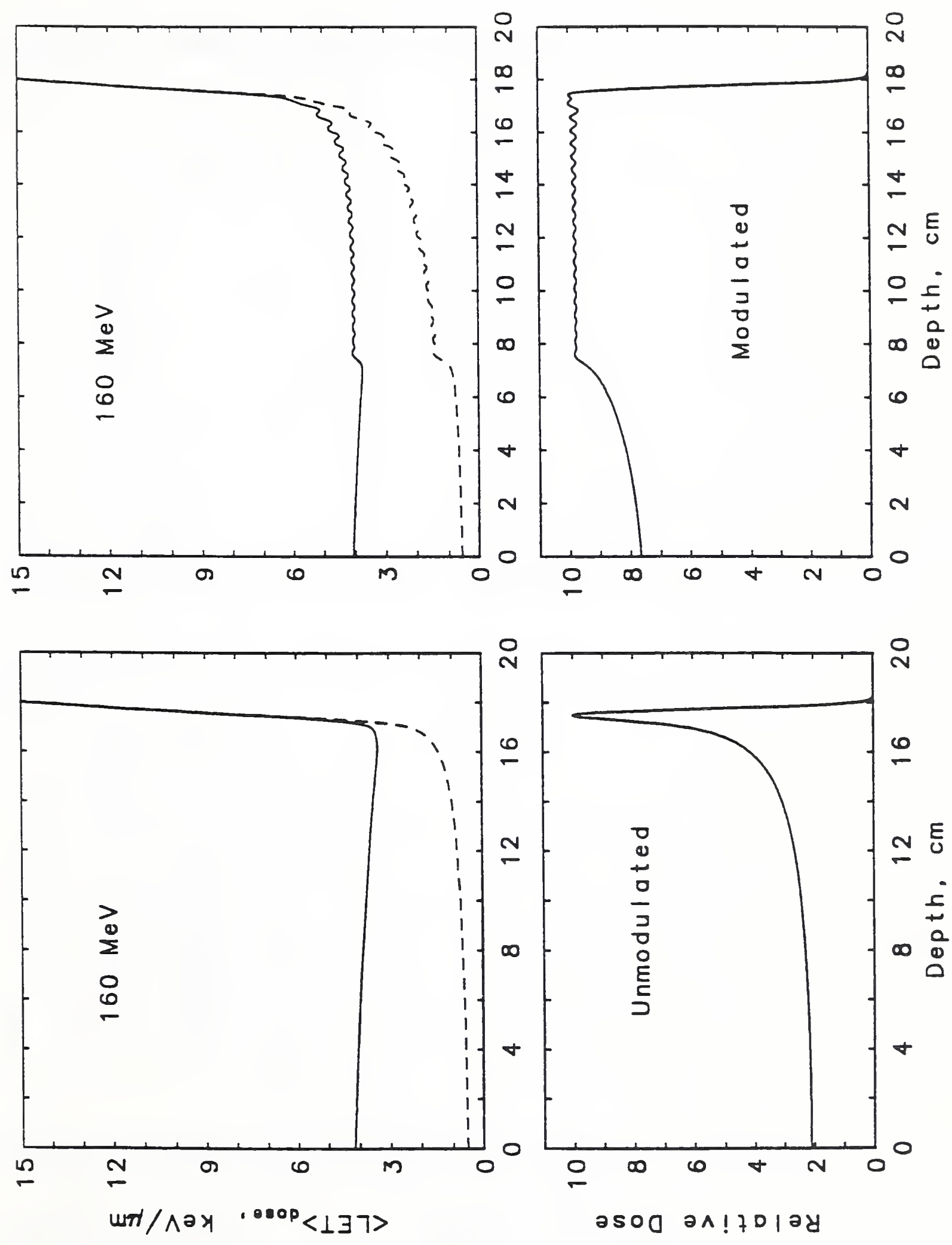

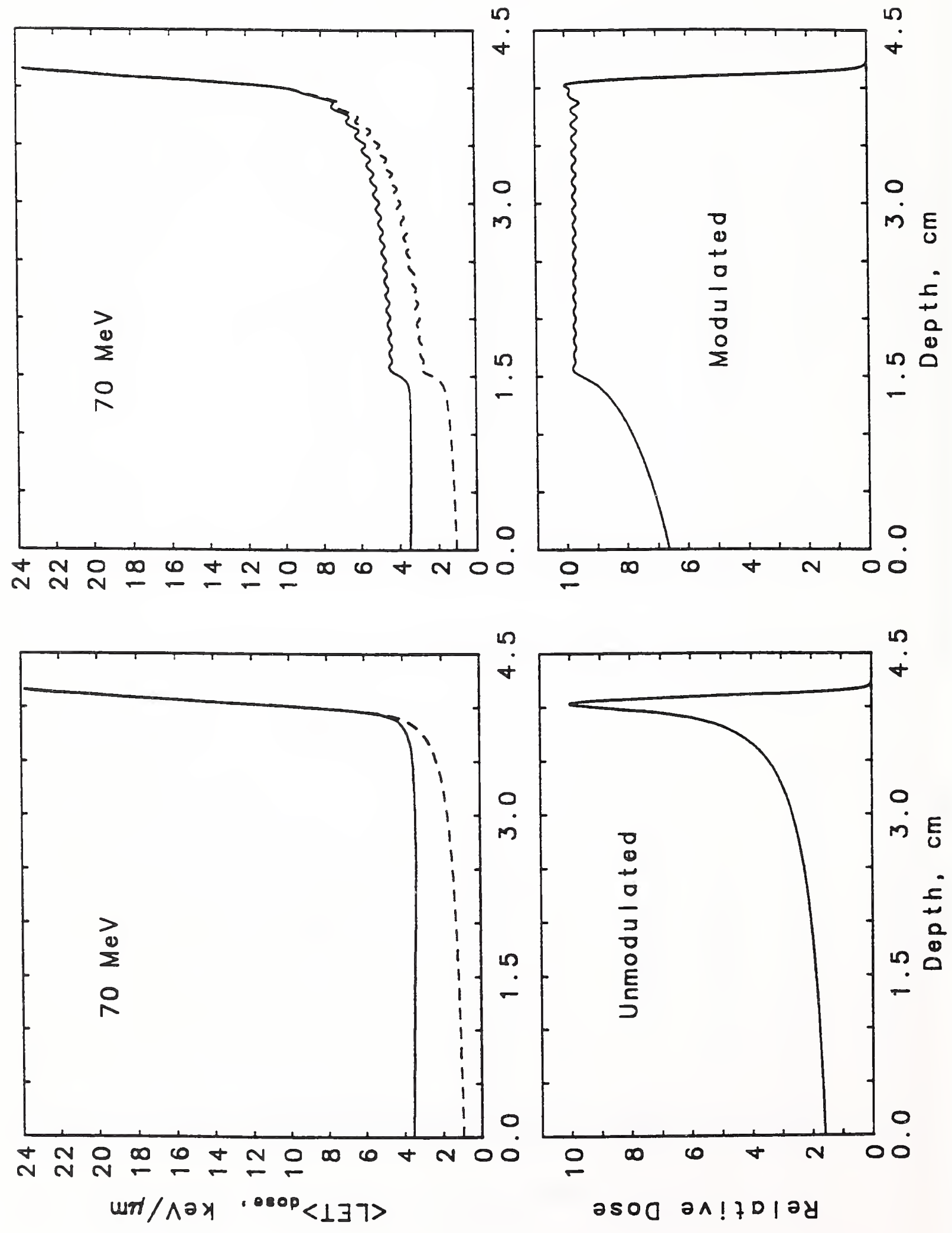


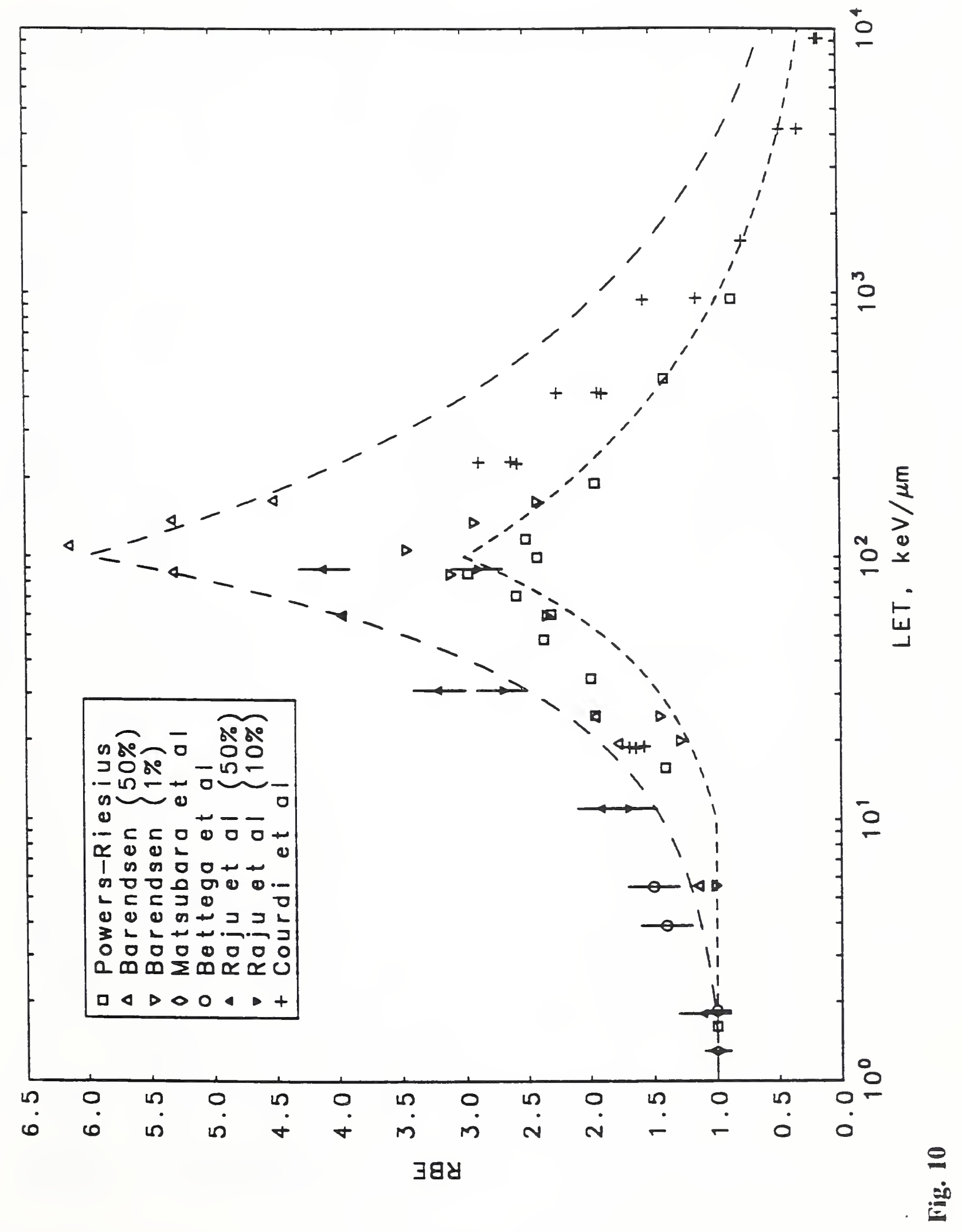




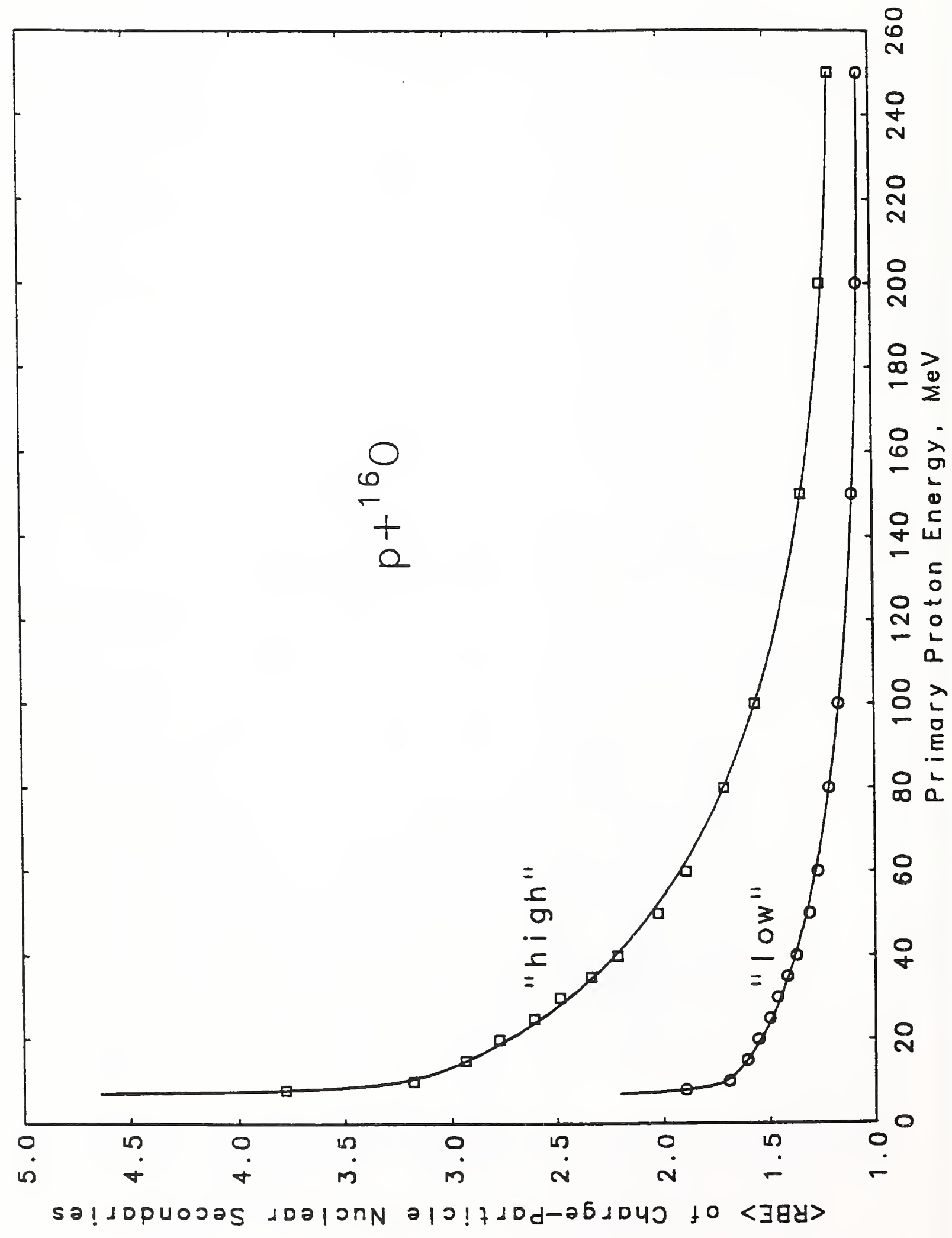




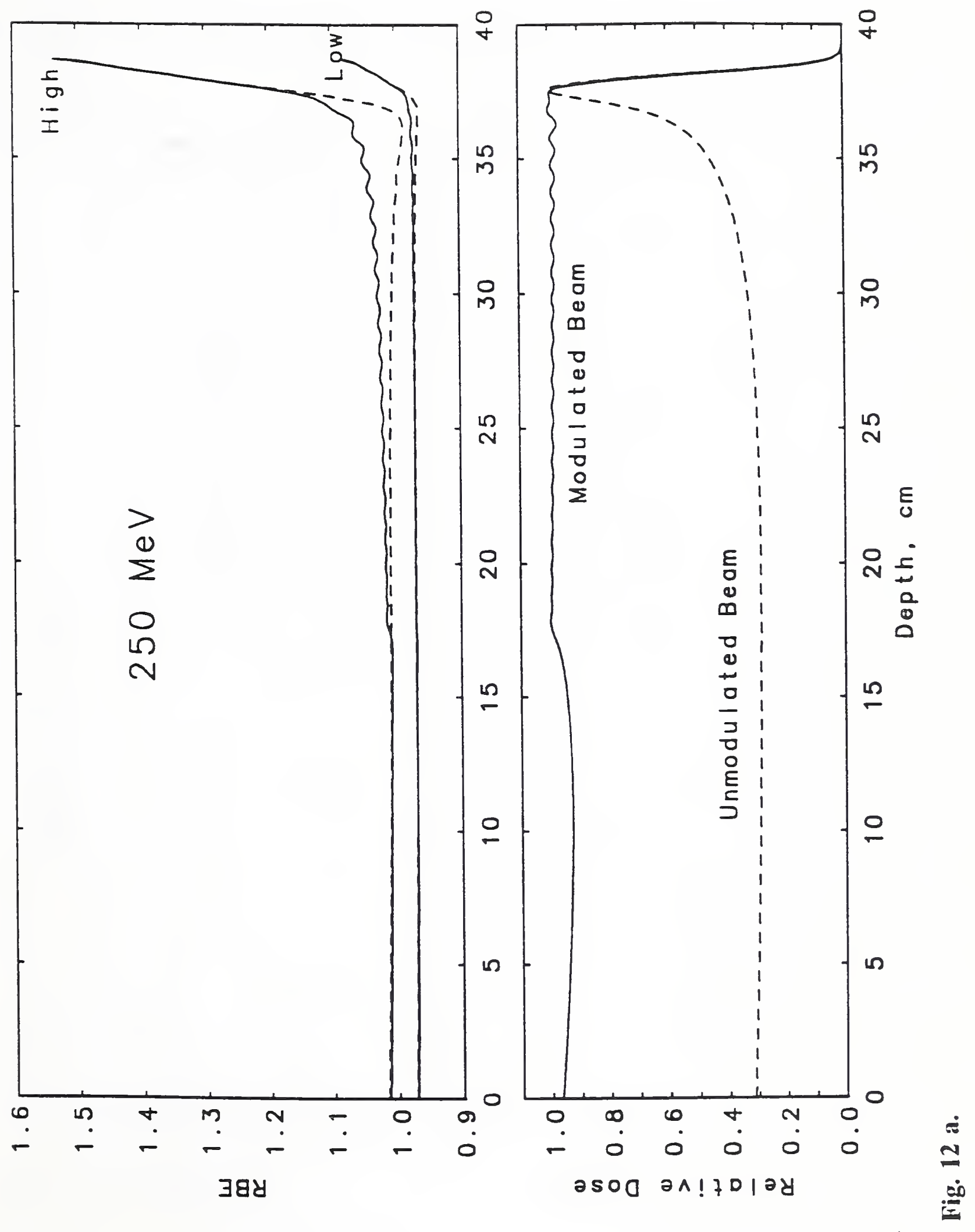




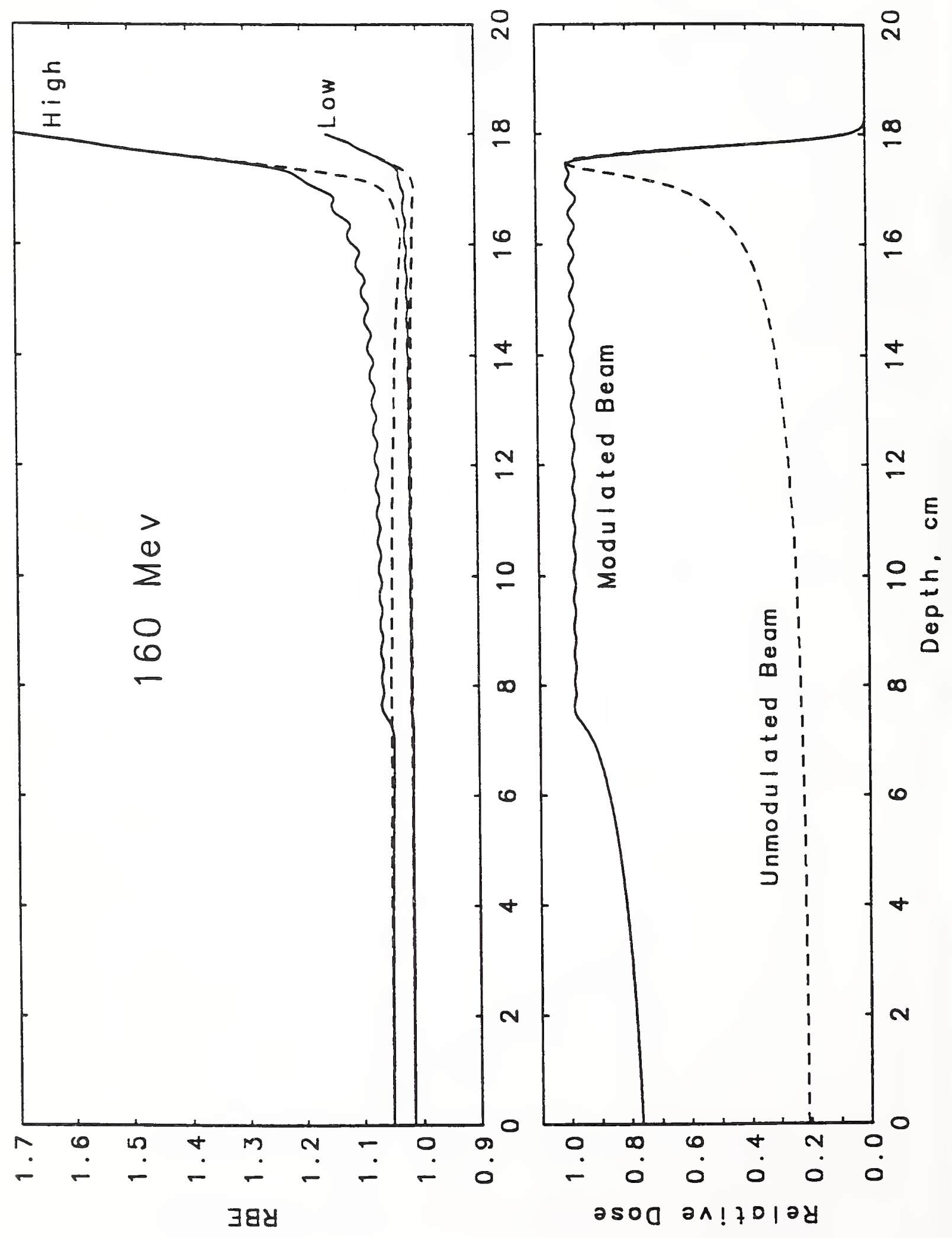




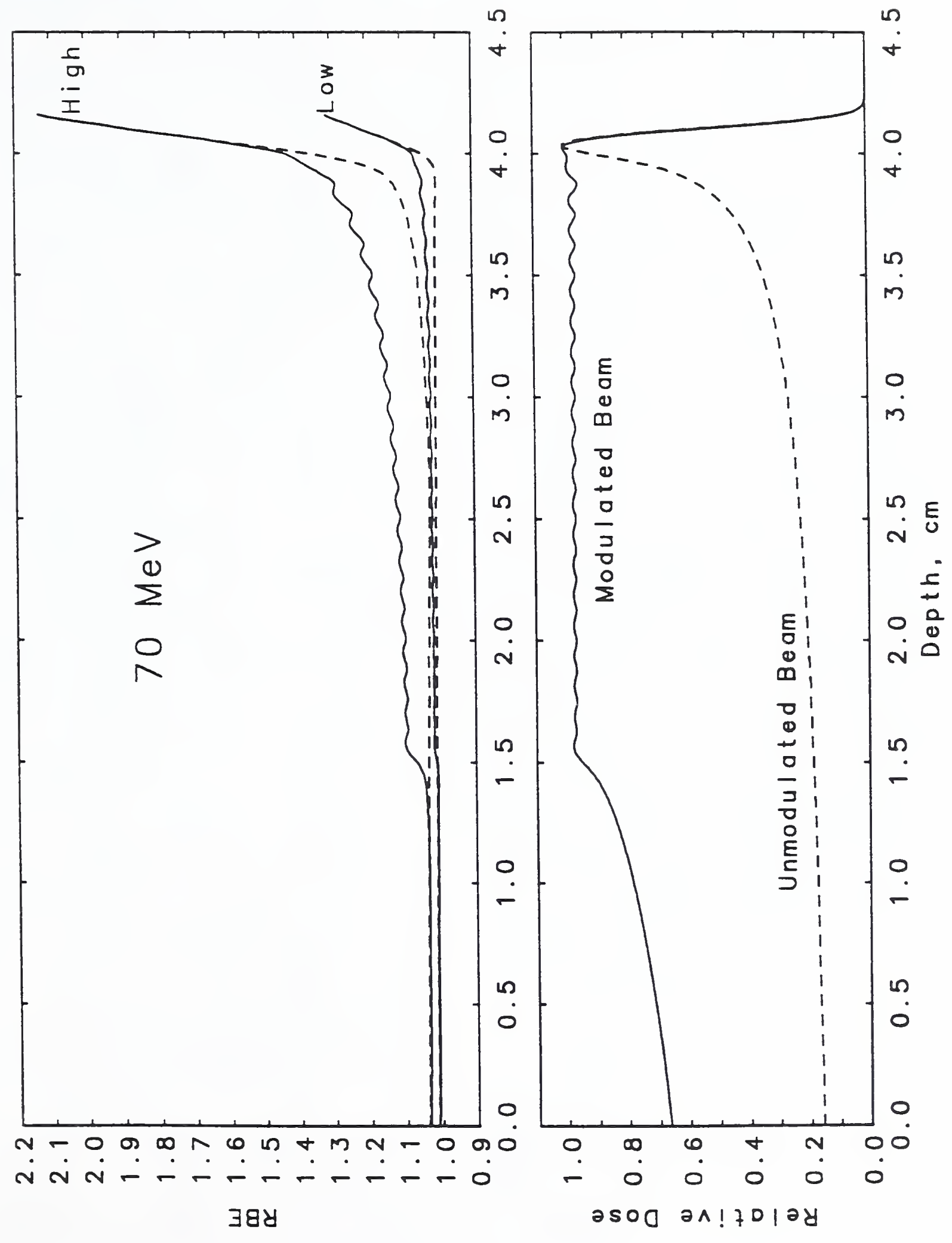




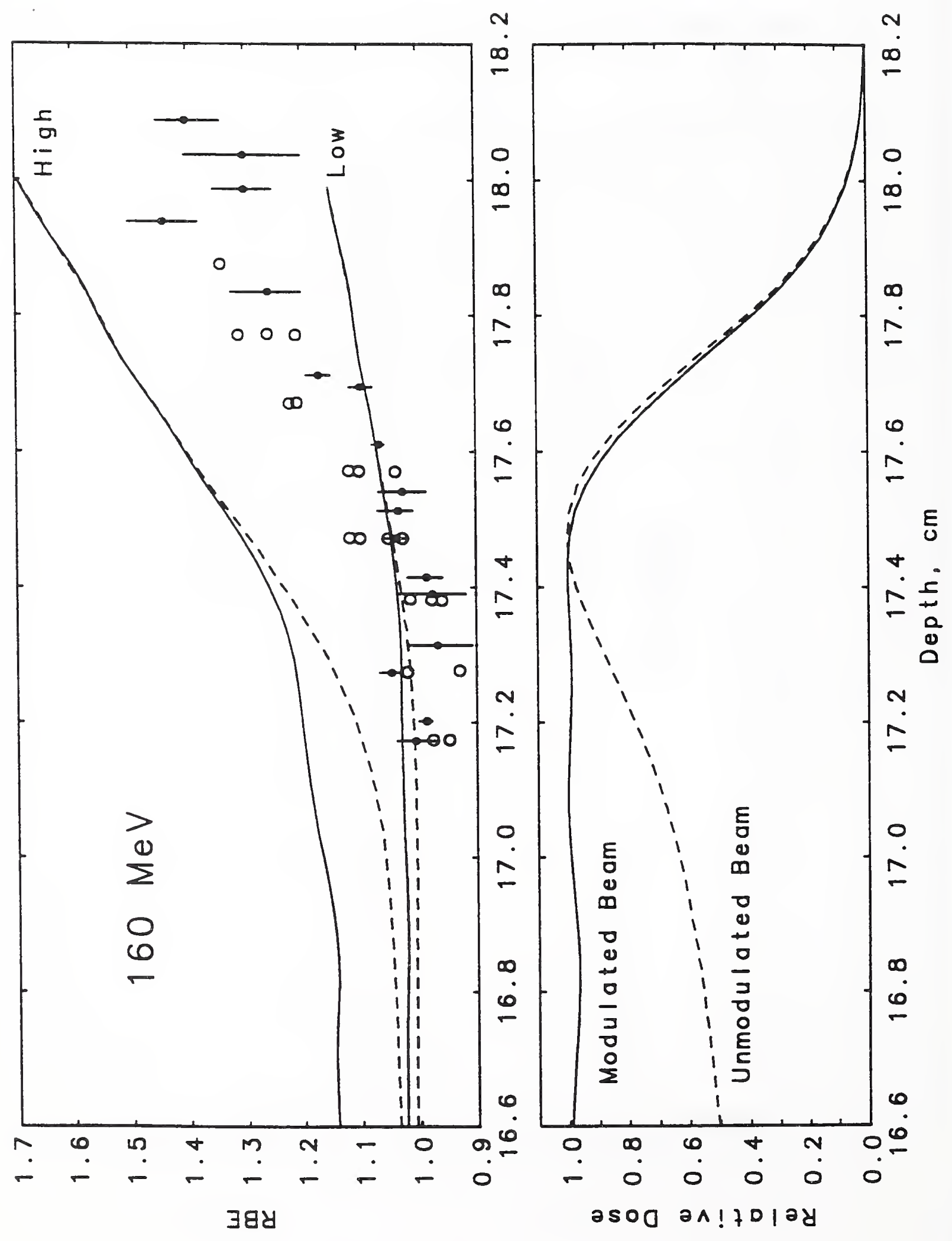

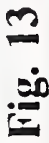



